\title{
SOLVABILITY OF ALGEBRAS OF PSEUDODIFFERENTIAL OPERATORS WITH PIECEWISE SMOOTH COEFFICIENTS ON SMOOTH MANIFOLDS
}

\author{
B. A. PLAMENEVSKII
}

\begin{abstract}
On a smooth compact manifold $\mathcal{M}$ without boundary, the $C^{*}$-algebra $\mathcal{A}$ generated on $L_{2}(\mathcal{M})$ by the operators of two classes is considered. One class consists of zero order pseudodifferential operators with smooth symbols. The other class comprises the operators of multiplication by functions ("coefficients") that may have discontinuities along a given collection of submanifolds (with boundary) of various dimensions; the submanifolds may intersect under nonzero angles. The situation is described formally by a stratification of the manifold $\mathcal{M}$. All the equivalence classes of irreducible representations of $\mathcal{A}$ are listed with a detailed proof. A solving composition series in $\mathcal{A}$ is constructed. This is a finite sequence of ideals $\{0\}=$ $I_{-1} \subset I_{0} \subset \cdots \subset I_{N}=\mathcal{A}$ whose subquotients $I_{j} / I_{j-1}$ are isomorphic to algebras of continuous functions with compact values; such operator-valued functions are defined on locally compact spaces and tend to zero at infinity.
\end{abstract}

\section{§1. INTRODUCTION}

In this paper, by an algebra and a morphism we mean a $C^{*}$-algebra and a $*$-morphism, $\mathcal{B} H$ is the algebra of all bounded operators on a Hilbert space $H$, and $\mathcal{K} H$ is the ideal of compact operators; $\widehat{\mathcal{Q}}$ stands for the spectrum of an algebra $\mathcal{Q}$, which is the set of all equivalence classes of irreducible representations of $\mathcal{Q}$ endowed with the Jacobson topology.

We recall some definitions and results. Let $\mathcal{M}$ be a smooth compact $m$-dimensional manifold without boundary $(m \geq 1)$, and let $\mathcal{P}$ be the algebra generated on $L_{2}(\mathcal{M})$ by the zero order scalar pseudodifferential operators $(\psi$ do $)$ with smooth symbols. We assume that the principal symbols of such $\psi$ do's given on the cotangent bundle $T^{*}(\mathcal{M})$ are degree zero homogeneous functions on every fiber $T^{*}(\mathcal{M})_{x}, x \in \mathcal{M}$. The algebra $\mathcal{P}$ is irreducible and contains the ideal $\mathcal{K} L_{2}(\mathcal{M})$, while the quotient algebra $\mathcal{P} / \mathcal{K} L_{2}(\mathcal{M})$ is commutative. The map $P \mapsto \Phi$ extends to an isomorphism $\mathcal{P} / \mathcal{K} L_{2}(\mathcal{M}) \simeq C\left(S^{*}(\mathcal{M})\right)$, where $\Phi$ is the principal symbol of an operator $P$ and $S^{*}(\mathcal{M})$ is the cospherical bundle over $\mathcal{M}$. All irreducible representations of $\mathcal{P} / \mathcal{K} L_{2}(\mathcal{M})$ are one-dimensional, and the spectrum $\left(\mathcal{P} / \mathcal{K} L_{2}(\mathcal{M})\right)^{\wedge}$ (the maximal ideal space) can be identified with $S^{*}(\mathcal{M})$. The map $\left(\mathcal{P} / \mathcal{K} L_{2}(\mathcal{M})\right)^{\wedge} \ni \pi \mapsto P(\pi):=\pi[P]$ is implemented as the function $\pi \mapsto \Phi(\pi) \in \mathbb{C}$; here $[P]$ stands for the class of $P$ in $\mathcal{P} / \mathcal{K} L_{2}(\mathcal{M})$ and $\pi$ is a (one-dimensional) representation of the quotient algebra. The element $[P]$ is invertible (and $P$ is Fredholm) if and only if $\Phi \neq 0$ everywhere on $S^{*}(\mathcal{M})$.

Now we turn to the algebra $\mathcal{A}$ generated by the operators in $\mathcal{P}$ and the multiplications $a I$ by functions $x \mapsto a(x)$ with discontinuities. The quotient algebra $\mathcal{A} / \mathcal{K} L_{2}(\mathcal{M})$ turns

2000 Mathematics Subject Classification. Primary 46L45, 47G30.

Key words and phrases. $C^{*}$-algebra, stratification, composition series, pseudodifferential operator.

Supported by grant NSh-816.2008.1. 
out to be noncommutative and acquires infinite-dimensional irreducible representations (for $\operatorname{dim} \mathcal{M}>1$ ). The map

$$
\left(\mathcal{A} / \mathcal{K} L_{2}(\mathcal{M})\right)^{\wedge} \ni \pi \mapsto A(\pi):=\pi[A]
$$

plays the role of the principal symbol of $A \in \mathcal{A}$; in contrast to the commutative case, not only scalars occur as $\pi[A]$ but also nontrivial operators on infinite-dimensional Hilbert spaces. As before, the "principal symbol" (1.1) provides a Fredholm criterion: an operator $A \in \mathcal{A}$ is Fredholm if and only if every operator $\pi(A)=\pi[A]$ for $\pi \in\left(\mathcal{A} / \mathcal{K} L_{2}(\mathcal{M})\right)^{\wedge}$ is invertible. Thus, to use this criterion, first we need to list all equivalence classes of irreducible representations of $\mathcal{A} / \mathcal{K} L_{2}(\mathcal{M})$ and indicate some implementations of such representations.

For the algebra $\mathcal{P}$ generated by $\psi$ do's with smooth symbols, we have

$$
\{0\} \subset \mathcal{K} L_{2}(\mathcal{M}) \subset \mathcal{P}, \quad \mathcal{P} / \mathcal{K} L_{2}(\mathcal{M}) \simeq C\left(S^{*}(\mathcal{M})\right) .
$$

In the general case, one can try to simplify the study of the noncommutative algebra $\mathcal{A} / \mathcal{K} L_{2}(\mathcal{M})$ by taking the quotient of $\mathcal{A} / \mathcal{K} L_{2}(\mathcal{M})$ by some ideal $J \supset \mathcal{K} L_{2}(\mathcal{M})$. The resulting loss of information is not large if the algebra $J / \mathcal{K} L_{2}(\mathcal{M})$ is comparatively simple.

Definition 1.1. An algebra $\mathcal{L}$ is said to be solvable if there is a composition series $\{0\}=I_{-1} \subset I_{0} \subset \cdots \subset I_{N}=\mathcal{L}$ of ideals $I_{j}$ such that the subquotients $I_{j} / I_{j-1}$ consist of continuous functions with compact values; more precisely, there exist isomorphisms

$$
I_{j} / I_{j-1} \simeq C_{0}\left(X_{j}\right) \otimes \mathcal{K} H_{j}, \quad j=0, \ldots, N<\infty,
$$

where $H_{j}$ is a Hilbert space, $X_{j}$ is a locally compact Hausdorff space, and $C_{0}\left(X_{j}\right)$ is the algebra of continuous functions tending to zero at infinity. A composition series with this property is said to be solving, and the number $N$ is called the length of the series.

The notion of a solvable algebra was suggested by A. S. Dynin [1. Examples in [1] were based on the papers by Gokhberg and Krupnik 2] (one-dimensional singular integral operators (SIO) with discontinuous coefficients), Boutet de Monvel [3] (SIO on a smooth compact manifold with boundary), and Douglas and Howe [4] (Wiener-Hopf operators on orthants); in this connection, we refer the reader to the survey [5] devoted to algebras of pseudodifferential operators with discontinuous symbols, the Wiener-Hopf algebras on "piecewise smooth" cones, and the Toeplitz algebras on bounded symmetric domains.

For example, assume that the coefficients $a$ in the algebra $\mathcal{A}$ are continuous everywhere except for a fixed point $x_{0} \in \mathcal{M}$; at that point, the coefficients may have discontinuities "of the first kind" (the limit $\lim a(x)$ as $x \rightarrow x_{0}$ exists and depends on the direction in which $x$ approaches $x_{0}$ ). The ideal com $\mathcal{A}$ spanned by the commutators of the elements in $\mathcal{A}$ is larger than $\mathcal{K} L_{2}(\mathcal{M})$. The algebra $\mathcal{A}$ is solvable, the shortest solving series is $\{0\} \subset$ $\mathcal{K} L_{2}(\mathcal{M}) \subset \operatorname{com} \mathcal{A} \subset \mathcal{A}$, and $\operatorname{com} \mathcal{A} / \mathcal{K} L_{2}(\mathcal{M}) \simeq C_{0}(\mathbb{R}) \otimes \mathcal{K} L_{2}\left(S^{m-1}\right), \mathcal{A} / \operatorname{com} \mathcal{A} \simeq$ $C\left(S^{*}(\mathfrak{C})\right)$, where $\mathfrak{C}$ is obtained by gluing the boundary (a sphere) to the manifold $\mathcal{M} \backslash x_{0}$.

Having a solving composition series, we can define a collection of symbols $\sigma_{1}(A), \ldots$, $\sigma_{N}(A)$ for an operator $A$ in a solvable algebra $\mathcal{L}$ (see 1). The symbol $\sigma_{j}(A)$ is an operator-valued function on the space $X_{j}$. The invertibility of $\sigma_{j}(A)$ at each point is necessary and sufficient for the invertibility of $A$ up to a summand in the ideal $I_{j}$. In terms of such symbols, the notions of the $j$-parametrix and $j$-index can be introduced for $A$. An important role is played by solving series in the study of the groups $K_{*}(\mathcal{L})$ of the operator $K$-theory related to a solvable algebra $\mathcal{L}[6]$.

In the paper, we consider the algebra $\mathcal{A}$ generated on a smooth compact manifold $\mathcal{M}$ by zero order pseudodifferential operators with smooth symbols and the operators of multiplication by functions with discontinuities "of the first kind" along a given collection 
of submanifolds. The main result is Theorem 3.13, which describes a solving composition series for $\mathcal{A}$. In fact, the entire paper is devoted to the proof of that theorem. Every ideal of the solving composition series is defined as the intersection of the kernels of certain explicitly indicated irreducible representations of the algebra $\mathcal{A}$. When verifying the properties of the series, we make use of the Jacobson spectral topology. Therefore, first we describe the irreducible representations of $\mathcal{A}$ and the topology of $\widehat{\mathcal{A}}$. The irreducible representations of $\mathcal{A}$ are listed in $\S 2$. Some preliminaries are given in $\S 3$, among which there is a description of the spectral topology (without proof). The remaining part of $\S 3$ contains the construction of the solving series and the verification of its properties.

A complete list of equivalence classes for irreducible representations of $\mathcal{A}$ was given in [7] without proof (there, the above-mentioned description of the spectral topology was justified). The paper 8 contains an outline of the construction of a solving composition series in $\mathcal{A}$ without verification of the properties of the series. The irreducible representations of an algebra of pseudodifferential operators on a piecewise smooth manifold were listed in [9] (with proof). Here we apply the method used in [9]; the extraction of that method from [9] would present a separate difficulty for the reader because of sophisticated objects studied in [9] (the paper [10] was devoted especially to the definition of pseudodifferential operators on piecewise smooth manifolds). In the present paper, we eliminate the gaps indicated.

\section{§. The algebra $\mathcal{A}$ And its irReducible Representations}

2.1. Stratification of the manifold $\mathcal{M}$. The algebra $\mathcal{A}$. Let $\mathcal{T}=\left\{s_{\alpha}^{p}\right\}$ be a finite partition of a manifold $\mathcal{M}$ into subsets (strata). In what follows, the coefficients can be discontinuous on strata of positive codimension. Any stratum $s_{\alpha}^{p}$ is a connected $p$ dimensional submanifold of $\mathcal{M}$ (possibly, nonclosed). The partition consisting of a single element (the manifold $\mathcal{M}$ itself) is not ruled out. The boundary $\bar{s}_{\alpha}^{p} \backslash s_{\alpha}^{p}$ of a stratum is the union of strata of smaller dimension (or is empty). The collection of all strata whose boundary contains $s_{\alpha}^{p}$ is called the $\operatorname{star} \operatorname{st}\left(s_{\alpha}^{p}\right)$ of $s_{\alpha}^{p}$.

Our stratifications $\mathcal{T}$ of $\mathcal{M}$ will satisfy some additional requirements. Namely, we assume that for any point $x \in s_{\alpha}^{p}$ there exist a neighborhood $U$ in $\mathcal{M}$ and a diffeomorphism $\varkappa$ of $U$ onto a neighborhood of the origin in $\mathbb{R}^{m}$ that locally rectifies $s_{\alpha}^{p}$ and all $s_{\beta}^{q} \in \operatorname{st}\left(s_{\alpha}^{p}\right)$; this means that $\varkappa\left(s_{\alpha}^{p} \cap U\right)$ is a neighborhood of the origin in $\mathbb{R}^{p}=\left\{y=\left(y_{1}, \ldots, y_{m}\right) \in \mathbb{R}^{m}: y_{1}=\cdots=y_{m-p}=0\right\}$ and $\varkappa\left(s_{\beta}^{q} \cap U\right)$ coincides near $\varkappa\left(s_{\alpha}^{p} \cap U\right)$ with the product $\mathbb{R}^{p} \times K^{q-p}$, where $K^{q-p}$ is a $(q-p)$-dimensional cone in the subspace $\mathbb{R}^{m-p}$ orthogonal to $\mathbb{R}^{p}$. Also, it will be assumed that $\varkappa(x)=0$ and $\varkappa^{\prime}(x)=1$. Let $S^{m-p-1}$ be the unit sphere in that subspace $\mathbb{R}^{m-p}$. We assume that, in their turn, the projections $\operatorname{pr}\left(s_{\beta}^{q}\right)$ of the sets $\varkappa\left(s_{\beta}^{q} \cap U\right)$ onto $S^{m-p-1}$, where $s_{\beta}^{q} \in \operatorname{st}\left(s_{\alpha}^{p}\right)$, form a partition of $S^{m-p-1}$ satisfying the above requirements; this partition will be denoted by $\mathcal{T}\left(s_{\alpha}^{p}\right)$ (the point $x$ is not indicated in the notation for simplicity). Under the given conditions the partition $\mathcal{T}$ of $\mathcal{M}$ is said to be admissible.

To the partition $\mathcal{T}\left(s_{\alpha}^{p}\right)$ and the stratum $\operatorname{pr}\left(s_{\beta}^{q}\right), q \leq m-1$, we give the roles of $\mathcal{T}$ and $s_{\alpha}^{p}$. This determines a partition $\mathcal{T}\left(s_{\alpha}^{p}, s_{\beta}^{q}\right)$ of the sphere $S^{m-q-1}$. In general, let $s_{0}, \ldots, s_{k}$ be a chain of strata such that $\operatorname{dim} s_{p}=d_{p}, d_{k} \leq m-1, s_{i} \in \operatorname{st}\left(s_{j}\right)$ for $i>j$ (simplifying the notation, we write strata with a single subscript). Continuing this procedure, we get partitions $\mathcal{T}\left(s_{0}\right), \mathcal{T}\left(s_{0}, s_{1}\right), \ldots, \mathcal{T}\left(s_{0}, \ldots, s_{k}\right)$. If $\mathcal{T}\left(s_{0}, \ldots, s_{k}\right)$ consists only of a single element, namely the sphere $S^{m-d_{k}-1}$ (for $d_{k}<m-1$ ), or of two points, i.e., the sphere $S^{0}$ (for $\left.d_{k}=m-1\right)$, then the chain $s_{0}, \ldots, s_{k}$ is said to be complete. 
Example 2.1. A partition $\mathcal{T}$ of $S^{2}$ is formed by a zero-dimensional stratum, i.e. the point $P_{+}=(0,0,1)$, and the two-dimensional stratum $s=S^{2} \backslash P_{+}$. It is clear that $\mathcal{T}\left(P_{+}\right)=S^{1}$. A complete chain is formed by $P_{+}$.

Example 2.2. A partition $\mathcal{T}$ of $S^{2}$ is formed by zero-dimensional strata, i.e. the points $P_{ \pm}=(0,0, \pm 1)$, a one-dimensional stratum, which is the meridian $\mu=\left\{x=\left(x_{1}, x_{2}, x_{3}\right)\right.$ : $\left.|x|=1, x_{1}>0, x_{2}=0\right\}$, and the two-dimensional stratum $s=S^{2} \backslash \bar{\mu}$. As a locally rectifying diffeomorphism in a neighborhood of the points $P_{ \pm}$or a point $x \in \mu$, we can take the orthogonal projection onto the tangent plane at the corresponding point. There are three complete chains: $P_{+}, \mu ; P_{-}, \mu$; and $\mu$. The chain $P_{+}, \mu$ generates a partition $\mathcal{T}\left(P_{+}\right)$consisting of two strata (the zero-dimensional stratum $p(\mu)$ and the one-dimensional $\left.S^{1} \backslash p(\mu)\right)$ and a partition $\mathcal{T}\left(P_{+}, \mu\right)=S^{0}$. The partition $\mathcal{T}(\mu)=S^{0}$ corresponds to the complete chain $\mu$.

Example 2.3. A partition $\mathcal{T}$ of a manifold $\mathcal{M}$ consists of an arbitrary $k$-dimensional $(k<m-1)$ smooth closed submanifold $\gamma$ and the stratum $\mathcal{M} \backslash \gamma$. Then $\mathcal{T}(\gamma)=S^{m-k-1}$.

We start our description of the generators of $\mathcal{A}$ from coefficients. On a manifold $\mathcal{M}$ endowed with an admissible partition $\mathcal{T}$, we introduce a class $\mathfrak{M}(\mathcal{T}) \equiv \mathfrak{M}(\mathcal{T}, \mathcal{M})$ of functions. The operators $a I$ with $a \in \mathfrak{M}(\mathcal{T})$ form one type of the generators of $\mathcal{A}$. We define the classes $\mathfrak{M}(\mathcal{T}, \mathcal{M})$ by induction on $\operatorname{dim} \mathcal{M}$. For $\operatorname{dim} \mathcal{M}=0$ such a class is the set of all functions on $\mathcal{M}$ (by $\mathcal{M}$ we mean a finite collection of points with discrete topology). Assume that the classes have been defined for $\operatorname{dim} \mathcal{M} \leq m-1$ and turn to the case where $\operatorname{dim} \mathcal{M}=m$. We suppose that $\mathcal{T}$ is an admissible partition of $\mathcal{M}$ and $|\mathcal{T}|$ is the union of all strata (as subsets of the manifold $\mathcal{M}$ ) of dimension no greater than $m-1$. By definition, a smooth function $f$ given on $\mathcal{M} \backslash|\mathcal{T}|$ belongs to the class $\mathfrak{M}(\mathcal{T}, \mathcal{M})$ if a representation of the form

$$
\left(f \cdot \varkappa^{-1}\right)(y, z)=\Psi(y, z)+\Psi_{1}(y, z)
$$

is valid in a neighborhood $U$ of any point of every stratum $s_{j}, d_{j} \leq m-1$; here $(y, z) \in$ $\varkappa(U) \subset \mathbb{R}^{m}, y=\left(y_{1}, \ldots, y_{m-d_{j}}\right), z=\left(z_{1}, \ldots, z_{d_{j}}\right), \Psi(y, z)=\Psi(y /|y|, z)$ for $y \neq 0$, and $\Psi(\cdot, z) \in \mathfrak{M}\left(\mathcal{T}\left(s_{j}\right), S^{m-d_{j}-1}\right)$ for all $z$; the terms in (2.1) depend continuously on $z$ in the norm of $L_{\infty}$ and $\Psi_{1}(y, z)=o(1)$ as $y \rightarrow 0$. The function $\Psi(\cdot, z)$ is called the limit value of $f$ at the point $z \in s_{j}$ (we write simply $z$ instead of $\varkappa^{-1}(0, z)$ ).

The other type of generators of the algebra $\mathcal{A}$ is presented by zero order pseudodifferental operators with smooth symbols; a more precise definition of this class of operators is as follows. Let $V$ be an open set in $\mathbb{R}^{m}$. We denote by $S_{0}(V)$ the set of functions in $C^{\infty}\left(V \times \mathbb{R}^{m}\right)$ that admit the representation $\sigma=\sigma_{0}+\sigma_{1}$, where $\sigma_{0}, \sigma_{1} \in C^{\infty}\left(V \times \mathbb{R}^{m}\right)$, $(x, \xi) \mapsto \sigma_{0}(x, \xi)$ is a homogeneous function of zero degree for large $|\xi|$, and $\sigma_{1}$ satisfies

$$
\sup \left\{\left|\partial^{\alpha} \partial^{\beta} \sigma_{1}(x, \xi)\right| ; x \in K, \xi \in \mathbb{R}^{m}\right\} \leq C(\alpha, \beta, K)\langle\xi\rangle^{-|\beta|-1}
$$

for all multiindices $\alpha, \beta$ and all compact sets $K \subset V$, while $\langle\xi\rangle=\left(1+|\xi|^{2}\right)^{1 / 2}$. By definition, a pseudodifferential operator $(\psi$ do $)$ of class $\Psi^{0}(V)$ is an operator of the form

$$
A u(x)=(2 \pi)^{-m / 2} \int e^{i x \xi} \sigma(x, \xi) \widehat{u}(\xi) d \xi,
$$

where $\sigma \in S_{0}(V), u \in C_{c}^{\infty}(V)$, and $\widehat{u}$ stands for the Fourier transform of $u$. The function $\sigma$ is called the symbol of $A$ and the function $\Phi$ defined by $\Phi(x, \xi)=\lim \sigma(x, t \xi)$ as $t \rightarrow+\infty$ is called the principal symbol of $A$. It is clear that $\xi \mapsto \Phi(x, \xi)$ is a homogeneous function of zero degree.

Now we introduce $\psi$ do's on a manifold $\mathcal{M}$. An operator $A: C^{\infty}(\mathcal{M}) \rightarrow C^{\infty}(\mathcal{M})$ is said to be in $\Psi^{0}(\mathcal{M})$ if for any coordinate neighborhood $\mathcal{V}$ and any diffeomorphism $\kappa: \mathcal{V} \rightarrow V \subset \mathbb{R}^{m}$, the operator $A_{\kappa}: u \mapsto[A(u \circ \kappa)] \circ \kappa^{-1}$ with $u \in C_{c}^{\infty}(V)$ belongs to 
$\Psi^{0}(V)$. The principal symbols $\Phi_{\kappa}$ of such $\psi$ do's for all charts $(\mathcal{V}, \kappa)$ are compatible in the sense that they determine a function $\Phi$ on the cotangent bundle $T^{*}(\mathcal{M})$ outside its zero section. The function $\Phi$ is homogeneous of zero degree on every fiber $T^{*}(\mathcal{M})_{x} \backslash 0$, $x \in \mathcal{M}$; it is called the principal symbol of $A \in \Psi^{0}(\mathcal{M})$. The operators in $\Psi^{0}(\mathcal{M})$ extend to continuous mappings $L_{2}(\mathcal{M}) \rightarrow L_{2}(\mathcal{M})$.

Thus, we assume that an admissible partition $\mathcal{T}$ is given on a smooth compact $m$ dimensional manifold $\mathcal{M}$ without boundary, and we let $\mathcal{A}$ stand for the algebra generated on $L_{2}(\mathcal{M})$ by all operators $a I$ with $a$ in $\mathfrak{M}(\mathcal{T}, \mathcal{M})$ and by all $\psi$ do's of class $\Psi^{0}(\mathcal{M})$.

2.2. The irreducible representations of the algebra $\mathcal{A}$ (formulation of a theorem). We suppose that the manifold $\mathcal{M}$ is endowed with a Riemannian metric. Let $s_{0}, \ldots, s_{k}$ be a chain of strata of the partition $\mathcal{T}$, i.e., $s_{i} \in \operatorname{st}\left(s_{j}\right)$ for $i>j, \operatorname{dim} s_{j}=d_{j}$, and $d_{k} \leq m-1$, where $m=\operatorname{dim} \mathcal{M}$. For the generators of $\mathcal{A}$ we define a localization procedure along this chain of strata.

Let $a \in \mathfrak{M}(\mathcal{T}, \mathcal{M})$, and let $a\left(z^{0} ; \cdot\right) \in \mathfrak{M}\left(\mathcal{T}\left(s_{0}\right), S^{m-d_{0}-1}\right)$ be the limit value of $a$ at a point $z^{0} \in s_{0}$. The $\left(d_{1}-d_{0}-1\right)$-dimensional stratum $\operatorname{pr}\left(s_{1}\right)$ of the partition $\mathcal{T}\left(s_{0}\right)$ corresponds to the stratum $s_{1}$. We denote by $a\left(z^{0}, z^{1} ; \cdot\right)$ the limit value of $a\left(z^{0} ; \cdot\right)$ at a point $z^{1} \in \operatorname{pr}\left(s_{1}\right)$. Continuing this process, we arrive at the collection $a\left(z^{0} ; \cdot\right), \ldots, a\left(z^{0}, \ldots, z^{k} ; \cdot\right)$, where $a\left(z^{0}, \ldots, z^{j} ; \cdot\right) \in \mathfrak{M}\left(\mathcal{T}\left(s_{1}, \ldots, s_{j}\right), S^{m-d_{j}-1}\right)$. We say that this collection is obtained by localizing $a$ along the chain $s_{0}, \ldots, s_{k}$. If the chain is complete, then $a\left(z^{0}, \ldots, z^{k} ; \cdot\right) \in C\left(S^{m-d_{k}-1}\right)$.

We extend the localization procedure to the generators of the other type, i.e., to the $\psi$ do's in $\Psi^{0}(\mathcal{M})$. Let $A \in \Psi^{0}(\mathcal{M})$, and let $\Phi$ be the principal symbol of $A$. In the tangent space $T(\mathcal{M})_{z^{0}}$ with $z^{0} \in s_{0}$, we introduce orthogonal coordinates $\left(x_{1}, \ldots, x_{m}\right)$, where the axes $x_{m-d_{0}+1}, \ldots, x_{m}$ are parallel to the stratum $s_{0}$. The symbol $\Phi\left(z^{0} ; \cdot\right)$ will be written in that coordinate system.

We introduce the operators

$$
A\left(z^{0} ; \theta^{0}\right)=F_{\eta \rightarrow x}^{-1} \Phi\left(z^{0} ; \eta, \theta^{0}\right) F_{y \rightarrow \eta} \in \mathcal{B} L_{2}\left(\mathbb{R}^{m-d_{0}}\right),
$$

where $\eta=\left(\eta_{1}, \ldots, \eta_{m-d_{0}}\right), \theta^{0}=\left(\theta_{1}, \ldots, \theta_{d_{0}}\right) \in \mathbb{R}^{d_{0}},\left|\theta^{0}\right|=1$, and $F$ is the Fourier transformation on $\mathbb{R}^{m-d_{0}}$; the operators (2.2) are not defined for $d_{0}=0$.

Put

$$
\mathfrak{A}_{\psi \rightarrow \varphi}\left(z^{0} ; \lambda\right)=E_{\omega \rightarrow \varphi}(\lambda)^{-1} \Phi\left(z^{0} ; \omega, 0\right) E_{\psi \rightarrow \omega}(\lambda) \in \mathcal{B} L_{2}\left(S^{m-d_{0}-1}\right),
$$

where $\varphi, \psi, \omega \in \mathbb{R}^{m-d_{0}},|\varphi|=|\psi|=|\omega|=1, \lambda \in \mathbb{R}$, and the operator $E(\lambda)$ is given by

$$
(E(\lambda) u)(\varphi)=(2 \pi)^{-n / 2} e^{i \frac{\pi}{2}(i \lambda+n / 2)} \Gamma(i \lambda+n / 2) \int_{S^{n-1}}(-\varphi \omega+i 0)^{-i \lambda-n / 2} u(\omega) d \omega
$$

for $n=m-d_{0}$. The operator $E(\lambda)$ is unitary on $L_{2}\left(S^{n-1}\right)$ for all $\lambda \in \mathbb{R}$, and

$$
\left(E(\lambda)^{-1} v\right)(\varphi)=(2 \pi)^{-n / 2} e^{i \frac{\pi}{2}(n / 2-i \lambda)} \Gamma(n / 2-i \lambda) \int_{S^{n-1}}(\varphi \omega+i 0)^{i \lambda-n / 2} v(\omega) d \omega ;
$$

see [11, Chapter 1]. We say that (2.2) and (2.3) are operators of the first generation.

Now we choose new Cartesian coordinates $\tilde{x}_{1}, \ldots, \tilde{x}_{m}$ in $T(\mathcal{M})_{z^{0}}$ with the same origin. The axes $\tilde{x}_{m-d_{0}+1}, \ldots, \tilde{x}_{m}$ coincide with the old $x_{m-d_{0}+1}, \ldots, x_{m}$. The axis $\tilde{x}_{m-d_{0}}$ is directed toward the point $z^{1} \in \operatorname{pr}\left(s_{1}\right), \operatorname{dim} \operatorname{pr}\left(s_{1}\right)=d_{1}-d_{0}-1 ;$ the axes $\tilde{x}_{m-d_{1}}, \ldots, \tilde{x}_{m-d_{0}-1}$ are parallel to the $\left(d_{1}-d_{0}-1\right)$-dimensional space tangent to the stratum $\operatorname{pr}\left(s_{1}\right)$ at $z^{1}$. The remaining axes $\tilde{x}_{1}, \ldots, \tilde{x}_{m-d_{1}-1}$ turn out to be parallel to the space tangent to $S^{n-1}$ and orthogonal to $\operatorname{pr}\left(s_{1}\right)$ at $z^{1}$. The coordinate transformation (with block-diagonal matrix) will be written in the form $x=\operatorname{diag}\left(J\left(z^{1}\right), I_{m-d_{0}}\right) \tilde{x}$. In what follows the old coordinates do not appear and the new ones are denoted by $x$. 
We introduce the operators of the "second generation":

$$
A\left(z^{0}, z^{1} ; \theta^{0}, \theta^{1}\right)=F_{\eta \rightarrow x}^{-1} \Phi\left(z^{0} ; J\left(z^{1}\right)\left(\eta, \theta^{1}\right), \theta^{0}\right) F_{y \rightarrow \eta} \in \mathcal{B} L_{2}\left(\mathbb{R}^{m-d_{1}}\right),
$$

where $\theta^{0} \in \mathbb{R}^{d_{0}}, \theta^{1} \in \mathbb{R}^{d_{1}-d_{0}},\left|\theta^{0}\right|^{2}+\left|\theta^{1}\right|^{2}=1$, and $F$ is the Fourier transformation on $\mathbb{R}^{m-d_{1}}$

$$
\mathfrak{A}\left(z^{0}, z^{1} ; \lambda\right)=E_{\omega \rightarrow \varphi}(\lambda)^{-1} \Phi\left(z^{0} ; J\left(z^{1}\right)(\omega, 0), 0\right) E_{\psi \rightarrow \omega}(\lambda) \in \mathcal{B} L_{2}\left(S^{m-d_{1}-1}\right),
$$

where $\lambda \in \mathbb{R}$, and $E(\lambda)^{ \pm 1}$ are given by (2.4), (2.5) for $n=m-d_{1}$. If $d_{0}=0$, then in (2.6) we define the operator

$$
A\left(z^{0}, z^{1} ; \theta^{1}\right)=F_{\eta \rightarrow x} \Phi\left(z^{0} ; J\left(z^{1}\right)\left(\eta, \theta^{1}\right)\right) F_{y \rightarrow \eta}
$$

instead of $A\left(z^{0}, z^{1} ; \theta^{0}, \theta^{1}\right)$, and in (2.7) we define the operator

$$
\mathfrak{A}\left(z^{0}, z^{1} ; \lambda\right)=E_{\omega \rightarrow \varphi}(\lambda)^{-1} \Phi\left(z^{0} ; J\left(z^{1}\right)(\omega, 0)\right) E_{\psi \rightarrow \omega}(\lambda) .
$$

Continuing, we arrive at the collection $A\left(z^{0}, \ldots, z^{j} ; \theta^{0}, \ldots, \theta^{j}\right), \mathfrak{A}\left(z^{0}, \ldots, z^{j} ; \lambda_{j}\right)$ of operators, where $0 \leq j \leq k, \lambda_{j} \in \mathbb{R}, \theta^{0} \in \mathbb{R}^{d_{0}}, \theta^{j} \in \mathbb{R}^{d_{j}-d_{j-1}}$ for $j \geq 1$, and $\left|\theta^{0}\right|^{2}+\cdots+$ $\left|\theta^{j}\right|^{2}=1$. We say that this collection is obtained by localizing $A \in \Psi^{0}(\mathcal{M})$ along the chain $s_{0}, \ldots, s_{k}$.

Although the operators $A\left(z^{0}, \ldots, z^{j} ; \theta^{0}, \ldots, \theta^{j}\right), \mathfrak{A}\left(z^{0}, \ldots, z^{j} ; \lambda_{j}\right)$ are constructed for special coordinate systems, they are in fact independent of the arbitrariness in their definition. Indeed, suppose that we have chosen a chain of strata $s_{0}, \ldots, s_{j}$. Let us glue the strata $s_{j-1}, \ldots, s_{0}$ to $s_{j}$. The collection $\left(z^{0}, \ldots, z^{j}\right)$ determines a $d_{j}$-dimensional subspace $T\left(z^{0}, \ldots, z^{j}\right)$ of $T(\mathcal{M})_{z^{0}}$ tangent to $s_{j}$. The point $\left(\theta^{0}, \ldots, \theta^{j}\right)$ runs over the unit sphere $S^{*}\left(z^{0}, \ldots, z^{j}\right)$ in the cotangent space $T^{*}\left(z^{0}, \ldots, z^{j}\right)$. Let $\mathbb{R}^{m-d_{j}}\left(z^{0}, \ldots, z^{j}\right)$ denote the orthogonal complement of $T^{*}\left(z^{0}, \ldots, z^{j}\right)$ in $T(\mathcal{M})_{z^{0}}$ and let $S^{m-d_{j}-1}\left(z^{0}, \ldots, z^{j}\right)$ be the unit sphere in this orthogonal complement. Recall that the principal symbol $\Phi$ of $A \in \Psi^{0}(\mathcal{M})$ is defined on the bundle of nonzero cotangent vectors. The operators $\mathfrak{A}\left(z^{0}, \ldots, z^{j} ; \lambda_{j}\right)$ and $A\left(z^{0}, \ldots, z^{j} ; \theta^{0}, \ldots, \theta^{j}\right)$ act in $L_{2}\left(S^{m-d_{j}-1}\left(z^{0}, \ldots, z^{j}\right)\right)$ and $L_{2}\left(\mathbb{R}^{m-d_{j}}\left(z^{0}, \ldots, z^{j}\right)\right)$, respectively. This implies that these operators are invariant under the changes of coordinates.

The functions $a$ in $\mathfrak{M}(\mathcal{T}, \mathcal{M})$ are smooth on $\mathcal{M} \backslash|\mathcal{T}|$. The set $\mathcal{M} \backslash|\mathcal{T}|$ is embedded in a compact set $\mathfrak{C}$, and the functions $a$ extend to $\mathfrak{C}$ by continuity; now we describe $\mathfrak{C}$. First, we attach the boundary of a tubular neighborhood (in $\mathcal{M})$ of every $(m-1)$-dimensional stratum to $\mathcal{M} \backslash|\mathcal{T}|$ (distinct points must be glued to distinct ones). Denote the resulting set by $\mathfrak{C}_{m-1}$. The boundary of a tubular neighborhood in $\mathcal{M}$ of each $(m-2)$-dimensional stratum $s$ is cut by the $(m-1)$-dimensional strata of the star $\operatorname{st}(s)$. We attach this boundary to $\mathfrak{C}_{m-1}$ so as to identify it along the cut with the boundary of an already glued tubular neighborhood of an $(m-1)$-dimensional stratum (distinct points must be identified with distinct ones). We denote the new set by $\mathfrak{C}_{m-2}$. Descending to the strata of smaller dimensions, finally we obtain the compact set $\mathfrak{C}:=\mathfrak{C}_{0}$.

Let $p: \mathfrak{C} \rightarrow \mathcal{M}$ be the natural projection, and let $S^{*}(\mathfrak{C}):=p^{*} S^{*}(\mathcal{M})$, where $S^{*}(\mathcal{M})$ is the cospherical bundle over $\mathcal{M}$ and $p^{*} S^{*}(\mathcal{M})$ is the induced bundle. In what follows the one-dimensional representations of the algebra $\mathcal{A}$ will be parametrized by the points of $S^{*}(\mathfrak{C})$.

Now, we pass to listing the irreducible representations of $\mathcal{A}$. As before, let $s_{0}, \ldots, s_{k}$ be a chain of strata of the partition $\mathcal{T}, \operatorname{dim} s_{j}=d_{j}$. We introduce the following series of mappings for generators $A \in \Psi^{0}(\mathcal{M})$ and $a \in \mathfrak{M}(\mathcal{T}, \mathcal{M})$ of $\mathcal{A}$.

Mappings into $\mathcal{B} L_{2}\left(\mathbb{R}^{m-d_{j}}\left(z^{0}, \ldots, z^{j}\right)\right)$ :

$$
\begin{aligned}
& \pi\left(z^{0}, \ldots, z^{j} ; \theta^{0}, \ldots, \theta^{j}\right): A \mapsto A\left(z^{0}, \ldots, z^{j} ; \theta^{0}, \ldots, \theta^{j}\right) \in \mathcal{B} L_{2}\left(\mathbb{R}^{m-d_{j}}\left(z^{0}, \ldots, z^{j}\right)\right), \\
& \pi\left(z^{0}, \ldots, z^{j} ; \theta^{0}, \ldots, \theta^{j}\right): a \mapsto a\left(z^{0}, \ldots, z^{j} ; \cdot\right) I \in \mathcal{B} L_{2}\left(\mathbb{R}^{m-d_{j}}\left(z^{0}, \ldots, z^{j}\right)\right) I
\end{aligned}
$$


where $j=0, \ldots, k$ and the function $a\left(z^{0}, \ldots, z^{j} ; \cdot\right)$ is zero degree homogeneous on $\mathbb{R}^{m-d_{j}} \backslash 0$. For $d_{0}=0$ the mappings $\pi\left(z^{0} ; \theta^{0}\right)$ are absent; given a chain of strata, the mappings $\pi\left(z^{0}, \ldots, z^{j} ; \theta^{0}, \ldots, \theta^{j}\right)$ in (2.8) are exchanged for $\pi\left(z^{0}, z^{1}, \ldots, z^{j} ; \theta^{1}, \ldots, \theta^{j}\right)$, where, in particular, $\pi\left(z^{0}, \ldots, z^{j} ; \theta^{1}, \ldots, \theta^{j}\right) A=A\left(z^{0}, \ldots, z^{j} ; \theta^{1}, \ldots, \theta^{j}\right)$. In what follows, as a rule, we make no remarks related to the specificity of the case where $d_{0}=0$.

Mappings into $\mathcal{B} L_{2}\left(S^{m-d_{j}-1}\left(z^{0}, \ldots, z^{j}\right)\right)$ :

$$
\begin{aligned}
& \pi\left(z^{0}, \ldots, z^{j} ; \lambda_{j}\right): A \mapsto \mathfrak{A}\left(z^{0}, \ldots, z^{j} ; \lambda_{j}\right) \in \mathcal{B} L_{2}\left(S^{m-d_{j}-1}\left(z^{0}, \ldots, z^{j}\right)\right), \\
& \pi\left(z^{0}, \ldots, z^{j} ; \lambda_{j}\right): a \mapsto a\left(z^{0}, \ldots, z^{j} ; \cdot\right) I \in \mathcal{B} L_{2}\left(S^{m-d_{j}-1}\left(z^{0}, \ldots, z^{j}\right)\right),
\end{aligned}
$$

where $j=0, \ldots, k$ and $\lambda_{j} \in \mathbb{R}$.

Mappings into $\mathbb{C}$ :

$$
\begin{aligned}
& \pi(x, \xi): A \mapsto \Phi(p(x), \xi) \in \mathbb{C}, \\
& \pi(x, \xi): a \mapsto a(x),
\end{aligned}
$$

where $(x, \xi) \in S^{*}(\mathfrak{C})_{x}$, and $S^{*}(\mathfrak{C})_{x}$ is the fiber over $x \in \mathfrak{C}$ of the cospherical bundle $S^{*}(\mathfrak{C})$, $p: \mathfrak{C} \rightarrow \mathcal{M}$ is the projection, and $\Phi$ is the principal symbol of $A$.

The next theorem describes all (up to equivalence) irreducible representations of the algebra $\mathcal{A}$.

Theorem 2.4. The algebra $\mathcal{A}$ is irreducible and contains the ideal $\mathcal{K} L_{2}(\mathcal{M})$. The mappings (2.8) -2.10) extend to representations of the quotient algebra $\mathcal{A} / \mathcal{K} L_{2}(\mathcal{M})$. The representations (2.8) and (2.9) corresponding to all complete chains of strata of the partition $\mathcal{T}$ are irreducible and nonequivalent. Any irreducible representation $\pi$ of $\mathcal{A}$ with $\operatorname{dim} \pi>1$ is equivalent either to one of the representations (2.8), (2.9) or to the identity representation. Any one-dimensional representation of $\mathcal{A}$ coincides with one of those in (2.10).

It is known that the algebra $\mathcal{P}$ generated by the $\psi$ do's in $\Psi^{0}(\mathcal{M})$ is irreducible and contains the ideal $\mathcal{K} L_{2}(\mathcal{M})$ (see, e.g., [1] ). Since $\mathcal{P}$ is a subalgebra in $\mathcal{A}$, the algebra $\mathcal{A}$ is also irreducible and $\mathcal{K} L_{2}(\mathcal{M}) \subset \mathcal{A}$. Moreover, the algebras $\pi\left(z^{0}, \ldots, z^{j} ; \lambda\right) \mathcal{A}$ contain the subalgebras generated by the operators $\pi\left(z^{0}, \ldots, z^{j} ; \lambda\right) A$, where $A \in \Psi^{0}(\mathcal{M})$, and the coefficients $a\left(z^{0}, \ldots, z^{j} ; \cdot\right)$ are smooth on $S^{m-d_{j}-1}$. In other words, such an algebra is spanned on $L_{2}\left(S^{n-1}\right)$ by operators of the form

$$
\mathfrak{A}(\lambda)=E_{\omega \rightarrow \varphi}(\lambda)^{-1} \Phi(\omega) E_{\psi \rightarrow \omega}(\lambda) \in \mathcal{B} L_{2}\left(S^{n-1}\right)
$$

and by the operators of multiplication $a I$, where $a$ and $\Phi$ belong to $C^{\infty}\left(S^{n-1}\right)$ for $n=m-$ $d_{j}-1$. In [12] it was shown (see also [11, Proposition 5.3.1]) that this algebra is irreducible and contains the ideal $\mathcal{K} L_{2}\left(S^{n-1}(\mathcal{M})\right)$. Finally, the algebras $\pi\left(z^{0}, \ldots, z^{j} ; \theta^{0}, \ldots, \theta^{j}\right) \mathcal{A}$ contain the subalgebras spanned by the operators $\pi\left(z^{0}, \ldots, z^{j} ; \theta^{0}, \ldots, \theta^{j}\right) A$, where $A \in$ $\Psi^{0}(\mathcal{M})$, and by the coefficients $a\left(z^{0}, \ldots, z^{j} ; \cdot\right)$ smooth on $S^{m-d_{j}-1}$. Simplifying the notation, we can say that any such subalgebra $\mathcal{L}(\theta)$ is generated on $L_{2}\left(\mathbb{R}^{n}\right)$ by operators of the form

$$
A(\theta)=F_{\eta \rightarrow x}^{-1} \Phi(\eta, \theta) F_{y \rightarrow \eta}
$$

and by the coefficients $x \mapsto a(x /|x|)$, where $\Phi$ is a zero degree homogeneous function on $\mathbb{R}^{m}, F$ is the Fourier transformation on $\mathbb{R}^{n}, a \in C^{\infty}\left(S^{n-1}\right), \Phi \mid S^{m-1} \in C^{\infty}\left(S^{m-1}\right)$, and $|\theta|=1$. The irreducibility of $\mathcal{L}(\theta)$ and the inclusion $\mathcal{K} L_{2}\left(\mathbb{R}^{n}\right) \subset \mathcal{L}(\theta)$ were proved in [13. This and Theorem 2.4 lead to the inclusion $\mathcal{K} H_{\pi} \subset \pi(\mathcal{A})$ for any irreducible representation of $\mathcal{A}$; here $H_{\pi}$ is the space of the representation $\pi$. Thus, we arrive at the following assertion.

Corollary 2.5. The algebra $\mathcal{A}$ is of type I. 


\subsection{Proof of Theorem 2.4.}

2.3.1. Plan of the proof. The localization principle will be applied at various stages of the proof. We recall its formulation. Assume that all algebras under consideration are subalgebras of an algebra $\mathcal{L}$. Let $\mathcal{B}$ and $\mathcal{C}$ be such subalgebras; we suppose that $\mathcal{C}$ is commutative. Let $\mathcal{C} \rightarrow C_{0}(\widehat{\mathcal{C}})$ be the Gelfand isomorphism, $\mathcal{C} \ni c \mapsto \hat{c}(\cdot) \in C_{0}(\widehat{\mathcal{C}})$. For each point $x \in \widehat{\mathcal{C}}$ we set $\mathcal{I}_{x}=\{c \in \mathcal{C}: \hat{c}(x)=0\}$. Then $\mathcal{I}_{x}$ is a maximal ideal in $\mathcal{C}$. We take any algebra $\mathcal{A}$ containing both $\mathcal{B}$ and $\mathcal{C}$ and denote by $\mathcal{J}_{x}$ the ideal of $\mathcal{A}$ generated by $\mathcal{I}_{x}$, and by $\mathcal{B}_{x}$ the image of $\mathcal{B}$ under the canonical map $p_{x}: \mathcal{A} \rightarrow \mathcal{A} / \mathcal{J}_{x}$. The passage from $\mathcal{B}$ to $\mathcal{B}_{x}$ will be called localization at $x$; the algebras $\mathcal{B}_{x}$ are called the local algebras.

Let $\rho: \mathcal{B}_{x} \rightarrow \mathcal{B} H$ be an irreducible representation of the algebra $\mathcal{B}_{x}$ on a Hilbert space $H$. Then the composition $\rho \circ\left(p_{x} \mid \mathcal{B}\right)$ is an irreducible representation of $\mathcal{B}$. Since the mapping

$$
\widehat{\mathcal{B}}_{x} \rightarrow \widehat{\mathcal{B}}: \rho \mapsto \pi \equiv \rho \circ\left(p_{x} \mid \mathcal{B}\right)
$$

is an injection, we can identify the spectrum $\widehat{\mathcal{B}}_{x}$ with a subset of the spectrum $\widehat{\mathcal{B}}$. Thus, $\widehat{\mathcal{B}} \supset \bigcup_{x \in \widehat{\mathcal{C}}} \widehat{\mathcal{B}}_{x}$. The localization principle states that, under some additional conditions, the reverse inclusion also occurs. From now on we assume that $\mathcal{L}$ has a unit $e$. We shall use the following three versions of the localization principle.

Proposition 2.6. Suppose that subalgebras $\mathcal{B}$ and $\mathcal{C}$ of $\mathcal{L}$ satisfy the following conditions: 1) the algebra $\mathcal{C}$ is commutative and contains the unit $e ; 2) c_{1} b c_{2}=0$ if $b \in \mathcal{B}$ and $c_{1}, c_{2} \in \mathcal{C}$ satisfy $c_{1} c_{2}=0$. Then

$$
\widehat{\mathcal{B}}=\bigcup_{x \in \widehat{\mathcal{C}}} \widehat{\mathcal{B}}_{x}
$$

Proposition 2.7. Assume that subalgebras $\mathcal{B}$ and $\mathcal{C}$ of $\mathcal{B} H$ satisfy the following conditions: 1) $\mathcal{K} H \subset \mathcal{B}$; 2) the algebra $\mathcal{C}$ is commutative and contains the identity operator; 3) $[B, C]:=B C-C B \in \mathcal{K} H$ for all $B \in \mathcal{B}$ and $C \in \mathcal{C}$. Then

$$
\widehat{\mathcal{B}}=\bigcup_{x \in \widehat{\mathcal{C}}} \widehat{\mathcal{B}}_{x} \cup[I d]
$$

Proposition 2.8. Let $J$ be an ideal of an algebra $\mathcal{A}$ and $\mathcal{C}$ a commutative subalgebra of A. Suppose that

(i) $\pi \mid \mathcal{C} \neq 0$ for every $\pi \in \widehat{\mathcal{A}}$;

(ii) for any $x_{1}, x_{2} \in \widehat{\mathcal{C}}$ with $x_{1} \neq x_{2}$ there exist $c_{1}, c_{2} \in \mathcal{C}$ such that $\hat{c}_{j}\left(x_{j}\right) \neq 0, j=1,2$, and $c_{1} \mathcal{A} c_{2} \subset J$

(iii) for any $\pi \in \widehat{J}$ and $x \in \widehat{\mathcal{C}}$ there exist $a \in J$ and $c \in \mathcal{I}_{x}$ such that $\pi(a c) \neq 0$. Then

$$
\widehat{\mathcal{A}}=\bigcup_{x \in \widehat{\mathcal{C}}} \widehat{\mathcal{A}}_{x} \cup \widehat{J}
$$

and

$$
(\mathcal{A} / J)^{\wedge}=\bigcup_{x \in \widehat{\mathcal{C}}} \widehat{\mathcal{A}}_{x}
$$

Note that condition (i) is fulfilled automatically if the algebra $\mathcal{A}$ contains a unit $e \in \mathcal{C}$. Proofs of Propositions 2.6 and 2.7 can be found in [5]; Proposition 2.8 was proved in 9]. Similar forms of the localization principle were used by R. Douglas [14], A. S. Dynin [15], and I. B. Simonenko [16].

We apply the localization principle (Proposition 2.7) to the algebra $\mathcal{A}$, taking $C(\mathcal{M})$ as a localizing algebra. It turns out that the local algebra $\mathcal{A}_{x}$ at a point $x \in \mathcal{M} \backslash|\mathcal{T}|$ is isomorphic to $C\left(S^{*}(\mathcal{M})_{x}\right)$. If $z^{0}$ is a point of a $d_{0}$-dimensional stratum $s_{0}$ and $d_{0}>0$, 
then the local algebra $\mathcal{A}_{z^{0}}$ is isomorphic to the algebra of functions on $S^{d_{0}-1}$ with values in $\mathcal{B} L_{2}\left(\mathbb{R}^{m-d_{0}}\right)$, spanned by the functions $\theta^{0} \mapsto A\left(z^{0} ; \theta^{0}\right)=\pi\left(z^{0} ; \theta^{0}\right) A$ and by the operators of multiplication $a\left(z^{0} ; \cdot\right) I=\pi\left(z^{0} ; \theta^{0}\right) a$; here $A \in \Psi^{0}(\mathcal{M})$ and $a \in \mathfrak{M}(\mathcal{T}, \mathcal{M})$, while $A\left(z^{0} ; \theta^{0}\right)$ and $a\left(z^{0} ; \cdot\right)$ are defined as in Subsection 2.2 In particular, localization of $\mathcal{A}\left(z^{0} ; \theta^{0}\right)$ leads to the algebra $\mathfrak{S}\left(z^{0}\right)$ generated by the functions $\mathbb{R} \ni \lambda \mapsto \mathfrak{A}\left(z^{0}, \lambda\right)$ (see (2.3) $)$ and by the operators of multiplication by the coefficients $a\left(z^{0} ; \cdot\right) \mid S^{n-1}$, where $n=m-d_{0}$. The operations in $\mathfrak{S}\left(z^{0}\right)$ are pointwise, and the norm is given by

$$
\|\mathfrak{A}(\cdot)\|=\sup \left\{\left\|\mathfrak{A}(\lambda) ; \mathcal{B} L_{2}\left(S^{n-1}\right)\right\| ; \lambda \in \mathbb{R}\right\} .
$$

If $d_{0}=0$, then instead of the algebra $\mathcal{A}\left(z^{0} ; \theta^{0}\right)$ we have $\mathfrak{S}\left(z^{0}\right)$ even at the first step of localization. The algebras $\mathcal{A}\left(z^{0} ; \theta^{0}\right)$ and $\mathfrak{S}\left(z^{0}\right)$ are called algebras of the first generation. Localizing such algebras, we obtain algebras of the second generation (of the same type), and so on. The last generation consists of commutative algebras. In essence, a description of the dynasty of local algebras amounts to listing all the equivalence classes of irreducible representations of the algebra $\mathcal{A}$. We proceed to the study of the dynasty.

2.3.2. Localization of the algebra $\mathcal{A}$. Localizing the algebra $\mathcal{A}$, we shall apply Proposition 2.7 with $\mathcal{B}=\mathcal{A}$ and $\mathcal{C}=C(\mathcal{M})$; the role of the algebra $\mathcal{L}$, which appeared before Proposition 2.7, will be given to $\mathcal{A}$ itself.

First, we consider localization at a point $z \in \mathcal{M} \backslash|\mathcal{T}|$. Assume that $A \in \Psi^{0}(\mathcal{M})$, $a \in \mathfrak{M}(\mathcal{T}, \mathcal{M})$, and $\Phi$ is the principal symbol of $A$. The coefficients $a$ are continuous at $z$. For the generators of $\mathcal{A}$ we define the mapping

$$
\mathfrak{l}_{z}: a \mapsto a(z), \quad \mathfrak{l}_{z}: A \mapsto \Phi(z ; \cdot) \mid S^{*}(\mathcal{M})_{z},
$$

where $S^{*}(\mathcal{M})_{z}$ is the fiber over $z$ of the cospherical bundle $S^{*}(\mathcal{M})$. The next assertion describes the local algebra $\mathcal{A} / \mathcal{J}_{z}$; the proof is taken from [17].

Proposition 2.9. The mapping (2.13) extends to an isomorphism $\mathcal{A}_{z}=\mathcal{A} / \mathcal{J}_{z} \cong$ $C\left(S_{z}^{*}(\mathcal{M})\right)$.

Proof. The mapping $\mathfrak{l}_{z}$ is compatible with the algebraic operations, i.e., is linear, multiplicative, and commutes with involution. Therefore, it suffices to show that

$$
\inf \left\{\|B+J\| ; J \in \mathcal{J}_{z}\right\}=\left\|\mathfrak{l}_{z}(B) ; C\left(S_{z}^{*}\right)\right\|,
$$

where $B$ is any finite combination of generators of $\mathcal{A}$.

First we verify the inequality

$$
\inf \left\{\|B+J\| ; J \in \mathcal{J}_{z}\right\} \geq\left\|\mathfrak{l}_{z}(B) ; C\left(S_{z}^{*}\right)\right\| .
$$

Suppose that $V$ is a coordinate neighborhood of a point $z, V \subset \mathcal{M} \backslash|\mathcal{T}|$, and that $\chi \in C_{c}^{\infty}(V),|\chi| \leq 1, \chi(z)=1$. Note that $\mathfrak{l}_{z}(B)=\mathfrak{l}_{z}(\chi B \chi)$ and $\|B+J\| \geq\|\chi B \chi+\chi J \chi\|$. Therefore, we may assume the following: $V=\mathbb{R}^{m}, z=0$, the role of $\mathcal{A}$ is played by the algebra generated by operators of the form $\chi A \chi$, where $A \in \Psi^{0}\left(\mathbb{R}^{m}\right), \chi \in C_{c}^{\infty}\left(\mathbb{R}^{m}\right)$, and the role of $\mathcal{J}_{z}$ is given to the ideal $\mathcal{J}_{0}$ of $\mathcal{A}$ spanned by the functions $\chi$ such that $\chi(0)=0$. We rewrite (2.14) in the form

$$
\inf \left\{\|A+J\| ; J \in \mathcal{J}_{0}\right\} \geq \sup \left\{|\Phi(0 ; \xi)| ; \xi \in S^{m-1}\right\} .
$$

Let $A \in \Psi^{0}\left(\mathbb{R}^{m}\right)$, and let $\sigma$ be the symbol of $A$, so that $A=\operatorname{Op} \sigma$ and $\Phi(0 ; \xi)=$ $\lim _{t \rightarrow+\infty} \sigma(0, t \xi)$. For $t>0$ we introduce the operator $U_{t}: u(\cdot) \mapsto t^{n / 2} u(t \cdot)$, unitary on $L_{2}\left(\mathbb{R}^{n}\right)$. We have

$$
\left(U_{t} A U_{t}^{-1} u\right)(x)=(2 \pi)^{-n / 2} \int e^{i x \xi} \sigma(t x, \xi / t) \hat{u}(\xi) d \xi, \quad u \in C_{c}^{\infty}\left(\mathbb{R}^{n}\right) .
$$


Setting $A_{0}:=F^{-1} \Phi(0 ; \xi) F$, we see that $U_{t}(A+J) U_{t}^{-1} \rightarrow A_{0}$ in the strong operator topology as $t \rightarrow 0$ for any $J \in \mathcal{J}_{0}$. This implies the inequality

$$
\|A+J\| \geq\left\|A_{0}\right\|=\sup \left\{|\Phi(0 ; \xi)| ; \xi \in S^{m-1}\right\},
$$

which leads to (2.15).

To prove the reverse inequality, we set

$$
B u(x)=F_{\xi \rightarrow x}^{-1} \Phi(0 ; \xi)(1-\chi(\xi)) F_{y \rightarrow \xi} u(y),
$$

where $\chi \in C_{c}^{\infty}\left(\mathbb{R}^{n}\right), 0 \leq \chi \leq 1$, and $\chi(0)=1$. We also introduce $b_{1}(x, \xi)=\sigma(x, \xi)-$ $\sigma(0, \xi)$ and $b_{2}(x, \xi)=\sigma(0, \xi)-\Phi(0 ; \xi)(1-\chi(\xi))$. Then

$$
\chi(A-B) \chi=\chi\left(\mathrm{Op} b_{1}\right) \chi+\chi\left(\mathrm{Op} b_{2}\right) \chi .
$$

The operator $\chi\left(\mathrm{Op} b_{1}\right) \chi$ belongs to the ideal $\mathcal{J}_{0}$. In order to check the same for the second term on the right in (2.16), we note that $(1-\chi) K \in \mathcal{J}_{0}$ for any compact operator $K$. Hence, $\mathcal{K} L_{2}\left(\mathbb{R}^{m}\right) \cap \mathcal{J}_{0} \neq 0$. Since $\mathcal{J}_{0}$ is a nonzero ideal of an irreducible algebra, it is irreducible. Therefore, $\mathcal{K} L_{2}\left(\mathbb{R}^{m}\right) \subset \mathcal{J}_{0}$. The relation $\lim _{\xi \rightarrow \infty} b_{2}(x, \xi)=0$ shows that the operator $\chi\left(\mathrm{Op} b_{2}\right) \chi$ is compact; consequently, it belongs to $\mathcal{J}_{0}$. Using (2.16), we obtain $\chi(A-B) \chi \in \mathcal{J}_{0}$. Finally,

$$
\inf \|\chi A \chi+J\| \leq\|\chi B \chi\| \leq \sup |\Phi(0 ; \xi)(1-\chi(\xi))| \leq \sup |\Phi(0 ; \xi)| .
$$

Now, let $z$ be a point of the stratum $s$ with $\operatorname{dim} s=d>0$. We introduce orthogonal coordinates in the plane tangent to $\mathcal{M}$ at $z$, taking the axes $x_{1}, \ldots, x_{m-d}$ orthogonal to $s$. The tangent plane will be denoted simply by $\mathbb{R}^{m}$ and a point $x$ in that plane will be written in the form $x=\left(x^{(1)}, x^{(2)}\right)$, where $x^{(1)}=\left(x_{1}, \ldots, x_{m-d}\right)$ and $x^{(2)}=$ $\left(x_{m-d+1}, \ldots, x_{m}\right)$. For $a \in \mathfrak{M}(\mathcal{T}, \mathcal{M})$, we have the functions $a(z ; \cdot) \in \mathfrak{M}\left(\mathcal{T}(s), S^{m-d-1}\right)$ (see Subsection 2.2), where $S^{m-d-1}$ is the unit sphere in the subspace $\left\{x \in \mathbb{R}^{m}: x=\right.$ $\left.\left(x^{(1)}, 0\right)\right\}$ (i.e., in the subspace of the tangent space $T(\mathcal{M})_{z}$ orthogonal to $s$ ). We introduce the operators

$$
A=a\left(z ; x^{(1)} /\left|x^{(1)}\right|\right) F_{\xi \rightarrow x}^{-1} \Phi(\xi) F_{y \rightarrow \xi},
$$

where $F$ is the Fourier transformation on $\mathbb{R}^{m}$ and $\Phi$ is a zero degree homogeneous function smooth outside the origin.

Denote by $\mathcal{E}$ the algebra spanned by the operators of the form

$$
S^{d-1} \ni \theta \mapsto A(\theta)=a(z ; x /|x|) F_{\eta \rightarrow x}^{-1} \Phi(\eta, \theta) F_{y \rightarrow \eta} \in \mathcal{B} L_{2}\left(\mathbb{R}^{m-d}\right),
$$

with the norm $\|A(\cdot) ; \mathcal{E}\|=\sup \left\{\left\|A(\theta) ; \mathcal{B} L_{2}\left(\mathbb{R}^{m-d}\right)\right\|, \theta \in S^{d-1}\right\}$; here $F$ stands for the Fourier transformation on $\mathbb{R}^{m-d}$. Let $\mathcal{A}(z ; \theta)$ for $\theta \in S^{d-1}$ be the algebra spanned by the operators $A(\theta)$ on $L_{2}\left(\mathbb{R}^{m-d}\right)$.

Proposition 2.10. Let $z$ be a point of a d-dimensional stratum $s$, and let $d>0$. Then:

1) The algebra $\mathcal{A}_{z}$ is generated on $L_{2}\left(\mathbb{R}^{m}\right)$ by operators of the form (2.17).

2) The algebras $\mathcal{A}_{z}$ and $\mathcal{E}$ are isomorphic.

3) We have

$$
\widehat{\mathcal{A}}_{z}=\bigcup_{\theta \in S^{d-1}} \widehat{\mathcal{A}}(z ; \theta) .
$$

Proof. 1) Let $V$ be a coordinate neighborhood of $z$ in $\mathcal{M}$ and $\varkappa$ a coordinate diffeomorphism that projects $V$ into the tangent space to $\mathcal{M}$ at $z$ so that $\varkappa(z)=0, \varkappa^{\prime}(z)=I$, and $V \ni \zeta \mapsto \varkappa(\zeta)=\left(x^{(1)}, x^{(2)}\right)$. Assume also that $B$ is a product of finitely many generators of $\mathcal{A}$. The operator $B$ written in local coordinates will be denoted by $B_{\varkappa}$. For $t>0$ we introduce the unitary operator $U_{t}=t^{m / 2} u(t \cdot)$ on $L_{2}\left(\mathbb{R}^{m}\right)$. Finally, we take $\chi \in C_{c}^{\infty}(V)$ 
and consider $U_{t}(\chi B \chi)_{\varkappa} U_{t}^{-1}$. This expression is a product of finitely many operators of the form

$$
v \mapsto \int e^{i x \xi} a(t x) \sigma_{\varkappa}(t x, \xi / t) \widehat{v}(\xi) d \xi
$$

where $\sigma_{\varkappa}$ is the symbol of an operator in $\Psi^{0}(\mathcal{M})$ and $a$ is a function in $\mathfrak{M}\left(\mathcal{T}(s), S^{m-d-1}\right)$ (homogeneous of degree zero) written in local coordinates. Therefore, the strong limit

$$
Q_{0}:=\lim _{t \rightarrow 0} U_{t}(\chi B \chi)_{\varkappa} U_{t}^{-1}
$$

exists in $L_{2}\left(\mathbb{R}^{m}\right)$. The operator $Q_{0}$ is a product of finitely many operators of the form (2.17), and

$$
\left\|Q_{0}\right\| \leq|\chi(0)|^{2}\|Q\|
$$

where $Q=(\psi B \psi)_{\varkappa}, \psi \in C_{c}^{\infty}(V)$, and $\psi \chi=\chi$.

Let $\mathfrak{Q}$ be the algebra spanned by operators of the form $(\chi B \chi)_{\varkappa}$ on $L_{2}\left(\mathbb{R}^{m}\right)$. We denote by $\mathfrak{J}$ the ideal of $\mathfrak{Q}$ generated by the operators of multiplication by functions $\zeta \in C_{c}^{\infty}\left(\mathbb{R}^{m}\right)$ with $\zeta(0)=0$. The inequality $\left\|Q_{0}\right\| \leq|\chi(0)|^{2}\|Q\|$ implies that the mapping $Q \mapsto Q_{0}$ extends to an epimorphism $q: \mathfrak{Q} / \mathfrak{J} \rightarrow \mathfrak{Q}_{0}$, where $\mathfrak{Q}_{0}$ is the algebra spanned by operators of the form (2.17). We show that $q$ is an isomorphism. Assume that $\phi \in C_{c}^{\infty}\left(\mathbb{R}^{m}\right)$ and $\phi(0)=1$. Then $\phi Q_{0} \phi \in \mathfrak{Q}$ and $q:\left[\phi Q_{0} \phi\right] \mapsto Q_{0}$, where $\left[\phi Q_{0} \phi\right]$ is the residue class of $\phi Q_{0} \phi$ in $\mathfrak{Q} / \mathfrak{J}$. The relations $Q-\phi Q_{0} \phi \in \mathfrak{J}$ and $\left\|\phi Q_{0} \phi\right\| \leq|\phi|^{2}\left\|Q_{0}\right\|$ imply that $q$ is a monomorphism.

Let $\mathcal{J}_{z}$ be the ideal of $\mathcal{A}$ generated by the operators of multiplication by $\eta \in C(\mathcal{M})$ with $\eta(z)=0$. The isomorphism $\mathcal{A}_{z} \simeq \mathfrak{Q}_{0}$ is implemented by the mapping

$$
\mathcal{A}_{z}=\mathcal{A} / \mathcal{J}_{z} \ni[A] \mapsto\left[\left(\varkappa^{-1}\right)^{*}(\chi A \chi)(\varkappa)^{*}\right] \in \mathfrak{Q} / \mathfrak{J} \rightarrow \mathfrak{Q}_{0} ;
$$

the last arrow stands for the mapping $q, \chi \in C_{c}^{\infty}(V)$, and $\chi=1$ near $z$.

2) We denote by $\hat{u}\left(y^{(1)}, \xi^{(2)}\right)=F_{y^{(2)} \rightarrow \xi^{(2)}} u\left(y^{(1)}, y^{(2)}\right)$ the partial Fourier transform of $u$. The definition (2.17) shows that

$$
(A u)^{\wedge}\left(x^{(1)}, \xi^{(2)}\right)=a(z ; \varphi) F_{\xi^{(1)} \rightarrow x^{(1)}}^{-1} \Phi\left(\xi^{(1)}, \xi^{(2)}\right) F_{y^{(1)} \rightarrow \xi^{(1)}} \hat{u}\left(y^{(1)}, \xi^{(2)}\right),
$$

where $\varphi=x^{(1)} /\left|x^{(1)}\right|$. We set $X=x^{(1)}\left|\xi^{(2)}\right|$ and $Y=y^{(1)}\left|\xi^{(2)}\right|$. Since $\Phi$ is a homogeneous function, we can rewrite (2.20) in the form

$$
\begin{aligned}
& (A u)^{\wedge}\left(X\left|\xi^{(2)}\right|^{-1}, \xi^{(2)}\right) \\
& \quad=(2 \pi)^{-(m-d)} a(z ; \varphi) \int_{\mathbb{R}^{m-d}} e^{i X \eta} \Phi(\eta, \theta) d \eta \int_{\mathbb{R}^{m-d}} e^{-i Y \eta} \hat{u}\left(Y\left|\xi^{(2)}\right|^{-1}, \xi^{(2)}\right) d Y .
\end{aligned}
$$

We shall write $x, y\left(\in \mathbb{R}^{m-d}\right)$ instead of $X, Y$. Let $v(y)$ and $A(\theta) v(x)$ stand for $\hat{u}\left(Y\left|\xi^{(2)}\right|^{-1}, \xi^{(2)}\right)$ and $(A u)^{\wedge}\left(X\left|\xi^{(2)}\right|^{-1}, \xi^{(2)}\right)$. Now identity (2.21) takes the form

$$
A(\theta) v(x)=a(z ; \varphi) F_{\eta \rightarrow x}^{-1} \Phi(\eta, \theta) F_{y \rightarrow \eta} v(y), \quad \theta \in S^{d-1} .
$$

Since the transformation $F_{y^{(2)} \rightarrow \xi^{(2)}}$ is unitary on $L_{2}\left(\mathbb{R}^{d}\right)$, we see that $\mathcal{A}_{z^{0}}$ and $\mathcal{E}$ are isomorphic.

3) We introduce the algebra $\mathcal{C}$ generated by the operator-valued functions

$$
S^{d-1} \ni \theta \mapsto c(\theta) I \in \mathcal{B} L_{2}\left(\mathbb{R}^{m-d}\right),
$$

where $c \in C\left(S^{d-1}\right)$ and $I$ is the identity operator on $L_{2}\left(\mathbb{R}^{m-d}\right)$. The algebra $\mathcal{C}$ is equipped with the norm

$$
\|c(\cdot) I ; \mathcal{C}\|=\sup \left\{\left\|c(\theta) I ; \mathcal{B} L_{2}\left(\mathbb{R}^{m-d}\right)\right\| ; \theta \in S^{d-1}\right\}=\left\|c ; C\left(S^{d-1}\right)\right\| .
$$

The algebra $\mathcal{E}$ does not contain $\mathcal{C}$; therefore we introduce the algebra $\mathcal{L}$ spanned by $\mathcal{C}$ and $\mathcal{E}$. We take $\mathcal{C}, \mathcal{E}$, and $\mathcal{L}$ for the roles of $\mathcal{C}, \mathcal{B}$, and $\mathcal{L}$ in Proposition 2.6. respectively. Since 
the elements of $\mathcal{C}$ commute with all elements of $\mathcal{L}$, the assumptions of the proposition are fulfilled, and we obtain (2.19).

Finally, we consider the case of $z \in s$, where $s$ is a point, i.e., a zero-dimensional stratum. This will complete the first stage of localization, namely, the localization of $\mathcal{A}$ with the localizing algebra $C(\mathcal{M})$.

Proposition 2.11. Let $z=s$ be a zero-dimensional stratum of the partition $\mathcal{T}$ of $\mathcal{M}$. Then:

1) The local algebra $\mathcal{A}_{z}$ is generated on $L_{2}\left(\mathbb{R}^{m}\right)$ by operators of the form

$$
A=a(z ; \varphi) F_{\xi \rightarrow x}^{-1} \Phi(\xi) F_{y \rightarrow \xi},
$$

where $\varphi=x /|x|, a(z ; \cdot) \in \mathfrak{M}\left(\mathcal{T}(s), S^{m-1}\right), F$ is the Fourier transformation on $\mathbb{R}^{m}$, and $\Phi$ is a homogeneous function of degree zero.

2) The algebra $\mathcal{A}_{z}$ is isomorphic to the algebra $\mathfrak{S}(z)$ spanned by the operator-valued functions $\mathbb{R} \ni \lambda \mapsto \mathfrak{A}(z ; \lambda) \in \mathcal{B} L_{2}\left(S^{m-1}\right)$, with the norm

$$
\|\mathfrak{A}(\cdot)\|=\sup \left\{\left\|\mathfrak{A}(\lambda) ; \mathcal{B} L_{2}\left(S^{m-1}\right)\right\| ; \lambda \in \mathbb{R}\right\} ;
$$

here

$$
\mathfrak{A}_{\psi \rightarrow \varphi}(z ; \lambda)=a(z ; \varphi) E_{\omega \rightarrow \varphi}(\lambda)^{-1} \Phi(\omega) E_{\psi \rightarrow \omega}(\lambda),
$$

and the operators $E(\lambda)$ and $E(\lambda)^{-1}$ are defined by (2.4) and (2.5) $($ for $n=m)$.

Proof. Assertion 1) can be checked by an evident modification of the first part of the proof of Proposition 2.10, In essence, assertion 2) is contained in [10, Chapter 2].

2.3.3. Localization of the algebra $\mathcal{A}(z ; \theta)$. In order to apply the localization principle (Proposition 2.7), we take $C\left(\overline{\mathbb{R}}^{n}\right)$ as a localizing algebra, where $n=m-d_{0}$ and $\overline{\mathbb{R}}^{n}$ is obtained by adjoining to $\mathbb{R}^{n}$ the sphere $S_{\infty}^{n-1}$ at infinity. The verification of the assumptions of Proposition 2.7 is based on Propositions 2.12 2.14 below, which will also be useful for some other goals. Propositions 2.12 and 2.13 were proved in [13, and Proposition 2.14 follows from [18, Theorem C].

Let $a$ and $\Phi$ be zero degree homogeneous functions on $\mathbb{R}^{n}$ and $\mathbb{R}^{m}$ (respectively) smooth outside the origin. Denote by $\mathcal{L}(\theta)$ the algebra generated on $L_{2}\left(\mathbb{R}^{n}\right)$ by operators of the form

$$
A(\theta)=F_{\eta \rightarrow x}^{-1} \Phi(\eta, \theta) F_{y \rightarrow \eta},
$$

where $|\theta|=1$, and by the operators $a I$ of multiplications by the functions $\mathbb{R}^{n} \ni x \mapsto$ $a(x /|x|)$.

Proposition 2.12. The algebra $\mathcal{L}(\theta)$ is irreducible; i.e., any subspace of $L_{2}\left(\mathbb{R}^{n}\right)$ invariant under $\mathcal{L}(\theta)$ is either 0 or $L_{2}\left(\mathbb{R}^{n}\right)$.

Proposition 2.13. Assume that $\Phi(\omega, 0) \equiv 0$, where $\omega \in S^{n-1}, A(\theta)$ is the operator (2.25), and $a$ is a zero degree homogeneous function on $\mathbb{R}^{n}$ smooth outside the origin. Then the commutator $[A(\theta), a]=A(\theta) a-a A(\theta)$ is compact on $L_{2}\left(\mathbb{R}^{n}\right)$.

It is known (see [19, 4.1.10]) that if an algebra $\mathcal{A}$ of operators on the Hilbert space $H$ is irreducible and $\mathcal{A} \cap \mathcal{K} H \neq 0$, then $\mathcal{K} H \subset \mathcal{A}$. Therefore, Propositions 2.12 and 2.13 imply that $\mathcal{K} L_{2}\left(\mathbb{R}^{n}\right) \subset \mathcal{L}(\theta)$.

Proposition 2.14. Let $A(\theta)$ be an operator of the form (2.25), and let $c \in C\left(\overline{\mathbb{R}}^{n}\right)$. Then the commutator $[A(\theta), c]$ is compact on $L_{2}\left(\mathbb{R}^{n}\right)$. 
Now we see that the hypotheses of Proposition 2.7 are fulfilled and turn to a description of local algebras. We slightly modify the notation to make it universal in what follows and to relate it to operators in the initial algebra $\mathcal{A}$. Recall that the algebra $\mathcal{A}\left(z^{0}, \theta^{0}\right)$ is generated on $L_{2}\left(\mathbb{R}^{n}\right)$ by operators of the form

$$
A\left(z^{0} ; \theta^{0}\right)=F_{\eta \rightarrow x}^{-1} \Phi\left(z^{0} ; \eta, \theta^{0}\right) F_{y \rightarrow \eta} \in \mathcal{B} L_{2}\left(\mathbb{R}^{n}\right)
$$

and the operators of multiplication by the coefficients

$$
\mathbb{R}^{n} \backslash 0 \ni x \mapsto a\left(z^{0} ; x /|x|\right), \quad a\left(z^{0} ; \cdot\right) \mid S^{n-1} \in \mathfrak{M}\left(\mathcal{T}\left(s_{0}\right), S^{n-1}\right)
$$

(compare with the notation in (2.18) $)$. On such generators, we define some mappings; below it will be shown that these mappings arise under localization at a point $z \in \mathbb{R}^{n}$. In items (i)-(v), the mapping $\mathfrak{l}(z)$ acts to the algebra $l_{z}$.

(i) For $z \in \mathbb{R}^{n} \backslash 0, z /|z| \in S^{n-1} \backslash\left|\mathcal{T}\left(s_{0}\right)\right|$, we set $l_{z}=C\left(S^{n-1}\right)$ and introduce the mapping

$$
\begin{aligned}
& \mathfrak{l}(z): a\left(z^{0} ; \cdot\right) \mapsto a\left(z^{0} ; z /|z|\right), \\
& \mathfrak{l}(z): A\left(z^{0} ; \theta^{0}\right) \mapsto \Phi\left(z^{0} ; \cdot, 0\right) .
\end{aligned}
$$
form

(ii) For $z=0$, denoting by $l_{z}$ the algebra generated on $L_{2}\left(\mathbb{R}^{n}\right)$ by the operators of the

$$
A\left(z^{0} ; 0\right)=F_{\eta \rightarrow x}^{-1} \Phi\left(z^{0} ; \eta, 0\right) F_{y \rightarrow \eta}
$$

and the operators of multiplication by the functions $\mathbb{R}^{n} \backslash 0 \ni x \mapsto a\left(z^{0} ; x /|x|\right)$, we introduce the mapping

$$
\begin{aligned}
& \mathfrak{l}(z): a\left(z^{0} ; \cdot\right) \mapsto a\left(z^{0} ; \cdot\right) I, \\
& \mathfrak{l}(z): A\left(z^{0} ; \theta^{0}\right) \mapsto A\left(z^{0} ; 0\right) .
\end{aligned}
$$

(iii) For $z \in S_{\infty}^{n-1}$ and $\varphi_{z} \in S^{n-1} \backslash\left|\mathcal{T}\left(s_{0}\right)\right|$, where $\varphi_{z}$ is the vector directed toward $z$, we set $l_{z}=C\left(\overline{\mathbb{R}}^{n}\right)$ and introduce the mapping

$$
\begin{aligned}
& \mathfrak{l}(z): a\left(z^{0} ; \cdot\right) \mapsto a\left(z^{0} ; \varphi_{z}\right), \\
& \mathfrak{l}(z): A\left(z^{0} ; \theta^{0}\right) \mapsto \Phi\left(z^{0} ; \cdot, \theta^{0}\right) .
\end{aligned}
$$

We pass to mappings that correspond to points $z \in \overline{\mathbb{R}}^{n} \backslash 0$ for $\varphi_{z} \in\left|\mathcal{T}\left(s_{0}\right)\right|$. Let $s_{1} \in \operatorname{st}\left(s_{0}\right), \operatorname{dim} s_{1}=d_{1}$, so that $\operatorname{pr}\left(s_{1}\right)$ is a $\left(d_{1}-d_{0}-1\right)$-dimensional stratum of the partition $\mathcal{T}\left(s_{0}\right)$ of the sphere $S^{n-1}$. We choose new coordinates $\tilde{x}_{1}, \ldots, \tilde{x}_{n}$ in $\mathbb{R}^{n}$ with the same origin. The axis $\tilde{x}_{n}$ is directed toward $\varphi_{z} \in \operatorname{pr}\left(s_{1}\right)$; the axes $\tilde{x}_{n-d_{1}+d_{0}}, \ldots, \tilde{x}_{n-1}$ are parallel to the space tangent to the stratum $\operatorname{pr}\left(s_{1}\right)$ at $\varphi_{z}$; the remaining axes are orthogonal to the stratum $\operatorname{pr}\left(s_{1}\right)$ at the point $\varphi_{z}$ and are parallel to the plane tangent to the sphere at that point. We write the coordinate transformation in the form $x=J(z) \tilde{x}$. Since the old coordinates will not appear in what follows, we denote the new ones by $x$. A point $x=\left(x_{1}, \ldots, x_{n}\right)$ will be written in the form $x=\left(x^{(1)}, x^{(2)}\right)$, where $x^{(1)}=$ $\left(x_{1}, \ldots, x_{n-\left(d_{1}-d_{0}\right)-1}\right)$ and $x^{(2)}=\left(x_{n-\left(d_{1}-d_{0}\right)}, \ldots, x_{n}\right)$.

(iv) For $z \in \mathbb{R}^{n} \backslash 0$ and $z /|z| \in \operatorname{pr}\left(s_{1}\right)$, let $l_{z}$ be the algebra generated on $L_{2}\left(\mathbb{R}^{n}\right)$ by operators of the form

$$
B\left(z^{0}, z ; 0\right)=F_{\xi \rightarrow x}^{-1} \Phi\left(z^{0} ; J(z) \xi, 0\right) F_{y \rightarrow \xi}
$$

and the operators of multiplication by the functions

$$
\mathbb{R}^{n} \backslash\left\{x=\left(x^{1}, x^{2}\right): x^{(2)}=0\right\} \ni x \mapsto a\left(z^{0}, z ; x\right)=a\left(z^{0}, z ; x^{1} /\left|x^{1}\right|\right) .
$$


(For brevity, we sometimes write $a\left(z^{0}, z ; x^{1} /\left|x^{1}\right|\right)$ instead of $a\left(z^{0}, z /|z| ; x^{1} /\left|x^{1}\right|\right)$.) We introduce the mapping

$$
\begin{aligned}
& \mathfrak{l}(z): a\left(z^{0} ; \cdot\right) \mapsto a\left(z^{0}, z ; \cdot\right) I, \\
& \mathfrak{l}(z): A\left(z^{0} ; \theta^{0}\right) \mapsto B\left(z^{0}, z ; 0\right) .
\end{aligned}
$$

(v) For $z \in S_{\infty}^{n-1}$ and $\varphi_{z} \in \operatorname{pr}\left(s_{1}\right)$, let $l_{z}$ denote the algebra generated on $L_{2}\left(\mathbb{R}^{n}\right)$ by operators of the form

$$
B\left(z^{0}, z ; \theta^{0}\right)=F_{\xi \rightarrow x}^{-1} \Phi\left(z^{0} ; J(z) \xi, \theta^{0}\right) F_{y \rightarrow \xi}
$$

and the operators of multiplication by the functions $x \mapsto a\left(z^{0}, z ; x^{1} /\left|x^{1}\right|\right)$; here we put $a\left(z^{0}, z ; x^{1} /\left|x^{1}\right|\right)=a\left(z^{0}, \varphi_{z} ; x^{1} /\left|x^{1}\right|\right)$. We introduce the mapping

$$
\begin{aligned}
& \mathfrak{l}(z): a\left(z^{0} ; \cdot\right) \mapsto a\left(z^{0}, z ; \cdot\right) I, \\
& \mathfrak{l}(z): A\left(z^{0} ; \theta^{0}\right) \mapsto B\left(z^{0}, z ; \theta^{0}\right) .
\end{aligned}
$$

Proposition 2.15. Let $\mathcal{A}\left(z^{0}, \theta^{0}\right)_{z}$ be the local algebra at $z \in \overline{\mathbb{R}}^{n}$ obtained by localization of $\mathcal{A}\left(z^{0}, \theta^{0}\right)$ with the localizing algebra $C\left(\overline{\mathbb{R}}^{n}\right)$. The mapping $\mathfrak{l}(z)$ defined on the generators of $\mathcal{A}\left(z^{0} ; \theta^{0}\right)$ by (2.26) -(2.30) extends up to an isomorphism $\mathfrak{l}(z): \mathcal{A}\left(z^{0} ; \theta^{0}\right)_{z} \rightarrow l_{z}$ for every $z \in \overline{\mathbb{R}}^{n}$.

Proof. Items (i) and (ii) can be treated in the same way as in Propositions 2.9 and 2.11 , respectively. We turn to the mapping (iii). We set

$$
A_{j k}(\theta)=F_{\eta \rightarrow x}^{-1} \Phi_{j k}(\eta, \theta) F_{y \rightarrow \eta},
$$

where $\Phi_{j k} \in C^{\infty}\left(S^{m-1}\right)$, and assume that $a_{j k}\left(z^{0} ; \cdot\right) \mid S^{n-1} \in \mathfrak{M}\left(\mathcal{T}\left(s_{0}\right), S^{n-1}\right)$ and $\chi_{j k} \in$ $C\left(\overline{\mathbb{R}}^{n}\right)$; the subscripts $j$ and $k$ run over finite sets. We introduce the unitary operator $U_{t} u(x)=u\left(x+t \varphi_{z}\right)$ on $L_{2}\left(\mathbb{R}^{n}\right)$ for $t \in \mathbb{R}$. Since the operators $A(\theta)=F_{\eta \rightarrow x}^{-1} \Phi(\eta, \theta) F_{y \rightarrow \eta}$ and $U_{t}$ commute, we have

$$
U_{t}\left(\sum_{j} \prod_{k} \chi_{j k} a_{j k} A_{j k}(\theta)\right) U_{t}^{-1} u=\left(\sum_{j} \prod_{k} U_{t}\left(\chi_{j k} a_{j k}\right) A_{j k}(\theta)\right) u .
$$

Clearly,

$$
\begin{aligned}
U_{t}\left(\chi_{j k}(x) a_{j k}\left(z^{0} ; x\right)\right) & =U_{t}\left(\chi_{j k}(x)\right) U_{t}\left(a_{j k}\left(z^{0} ; x\right)\right) \\
& =\chi_{j k}\left(x+t \varphi_{z}\right) a_{j k}\left(z^{0} ; x+t \varphi_{z}\right) \longrightarrow \chi_{j k}(z) a_{j k}\left(z^{0} ; \varphi_{z}\right)
\end{aligned}
$$

as $t \rightarrow+\infty$. Therefore, the following strong limit exists:

$$
\left.\sum_{j} \prod_{k} U_{t}\left(\chi_{j k} a_{j k}\right) A_{j k}(\theta) \longrightarrow \sum_{j} \prod_{k} \chi_{j k}(z) a_{j k}\left(z^{0} ; \varphi_{z}\right)\right) A_{j k}(\theta) .
$$

Together with (2.31), this shows that the operator $U_{t}\left(\sum_{j} \prod_{k} \chi_{j k} a_{j k} A_{j k}(\theta)+J\right) U_{t}^{-1}$ tends strongly to the same limit as $t \rightarrow+\infty$, where $J$ is any element in $\mathcal{J}_{z}$. Since $U_{t}$ is unitary and since the norm of a strong limit does not exceed the lower limit of the norms, we obtain

$$
\begin{aligned}
& \left\|\sum_{j} \prod_{k} \chi_{j k}(z) a_{j k}\left(\varphi_{z}\right) A_{j k}(\theta) ; \mathcal{B} L_{2}\left(\mathbb{R}^{n}\right)\right\| \\
& \quad \leq \inf _{J}\left\{\left\|\sum_{j} \prod_{k} \chi_{j k} a_{j k} A_{j k}(\theta)+J ; \mathcal{B} L_{2}\left(\mathbb{R}^{n}\right)\right\| ; J \in \mathcal{J}_{z}\right\} .
\end{aligned}
$$


Since the operators $a_{j k}\left(\varphi_{z}\right) A_{j k}(\theta)$ and $a_{j k}\left(z^{0} ; \cdot\right) A_{j k}(\theta)$ belong to the same residue class in the quotient algebra $\mathcal{A}\left(z^{0} ; \theta\right) / \mathcal{J}_{z}$, the reverse inequality is also true. Thus,

$$
\begin{aligned}
\inf _{J}\{ & \left.\left\|\sum_{j} \prod_{k} \chi_{j k} a_{j k}\left(z^{0} ; \cdot\right) A_{j k}(\theta)+J ; \mathcal{B} L_{2}\left(\mathbb{R}^{n}\right)\right\| ; J \in \mathcal{J}_{z}\right\} \\
& =\left\|\sum_{j} \prod_{k} \chi_{j k}(z) a_{j k}\left(z^{0} ; \varphi_{z}\right) A_{j k}(\theta) ; \mathcal{B} L_{2}\left(\mathbb{R}^{n}\right)\right\| \\
& =\left\|\sum_{j} \prod_{k} \chi_{j k}(z) a_{j k}\left(z^{0} ; \varphi_{z}\right) \Phi_{j k}(\cdot, \theta) ; C\left(\mathbb{R}^{n}\right)\right\| .
\end{aligned}
$$

It remains to set $\chi_{j k}=1$ and replace $\theta$ and $\Phi_{j k}(\cdot, \theta)$ by $\theta^{0}$ and $\Phi_{j k}\left(z^{0} ; \cdot, \theta^{0}\right)$.

Now we pass to the mapping (iv). Introducing the unitary operator $\left(U_{t} u\right)(x)=$ $t^{m / 2} u(z+t(x-z))$ on $L_{2}\left(\mathbb{R}^{n}\right)$ for $t>0$, we consider the composition $U_{t} Q U_{t}^{-1}$, where $Q$ is a product of finitely many generators of $\mathcal{A}\left(z^{0} ; \theta^{0}\right)$ and functions in $C\left(\overline{\mathbb{R}}^{n}\right)$. The properties of the coefficients imply the existence of the strong limit

$$
Q_{0}=\lim _{t \rightarrow 0} U_{t} Q U_{t}^{-1}
$$

Since

$$
\begin{aligned}
& \lim _{t \rightarrow 0}\left(U_{t} a\left(z^{0} ; \cdot\right) U_{t}^{-1} u\right)(x)=a\left(z^{0}, z ; x^{1} /\left|x^{1}\right|\right) u(x), \\
& \lim _{t \rightarrow 0}\left(U_{t} F_{\xi \rightarrow x}^{-1} \Phi\left(z^{0} ; J(z) \xi, \theta^{0}\right) F_{y \rightarrow \xi} U_{t}^{-1} u\right)(x)=\left(F_{\xi \rightarrow x}^{-1} \Phi\left(z^{0} ; J(z) \xi, 0\right) F_{y \rightarrow \xi} u\right)(x),
\end{aligned}
$$

the operators $Q_{0}$ generate on $L_{2}\left(\mathbb{R}^{n}\right)$ the algebra $l_{z}$ defined in (iv) before Proposition 2.15. Let $\mathfrak{Q}$ be the algebra spanned by $\mathcal{A}\left(z^{0} ; \theta^{0}\right)$ and $C\left(\overline{\mathbb{R}}^{n}\right)$. From (2.34) and (2.35) it follows that the mapping $Q \mapsto Q_{0}$ extends up to an epimorphism $q: \mathfrak{Q} / \mathfrak{J} \rightarrow l_{z}$, where $\mathfrak{J}$ is the ideal generated in $\mathfrak{Q}$ by the functions $\chi$ in $C\left(\overline{\mathbb{R}}^{n}\right)$ such that $\chi(z)=0$. We show that $q$ is an isomorphism. If the support of $\chi \in C_{c}^{\infty}\left(\mathbb{R}^{n}\right)$ belongs to a sufficiently small neighborhood of $z$, then $\chi Q_{0}$ is in $\mathfrak{Q}$, and if $\chi(z)=1$, then the operators $Q$ and $\chi Q_{0}$, where $Q_{0}=\lim _{t \rightarrow 0} U_{t} Q U_{t}^{-1}$, represent the same residue class $[Q]$ in $\mathfrak{Q} / \mathfrak{J}$. Hence, $\|[Q]\| \leq\left\|\chi Q_{0}\right\| \leq \sup |\chi|\left\|Q_{0}\right\|$, which means that $q$ is a monomorphism. Thus, $q$ is an isomorphism.

Finally, consider the mapping (v). We can argue as in the case of (iii). Namely, as $A_{j k}(\theta)$ we take operators of the form

$$
B_{j k}\left(z^{0}, z ; \theta^{0}\right)=F_{\xi \rightarrow x}^{-1} \Phi_{j k}\left(z^{0} ; J(z) \xi, \theta^{0}\right) F_{y \rightarrow \xi},
$$

and as coefficients we choose $a_{j k}\left(z^{0} ; \cdot\right)$, which are zero degree homogeneous functions satisfying $a_{j k}\left(z^{0} ; \cdot\right) \mid S^{n-1} \in \mathfrak{M}\left(\mathcal{T}\left(s_{0}\right), S^{n-1}\right)$. Then we have an identity similar to (2.31),

$$
\begin{aligned}
U_{t}( & \left.\sum_{j} \prod_{k} \chi_{j k} a_{j k}\left(z^{0} ; \cdot\right) B_{j k}\left(z^{0}, z ; \theta^{0}\right)\right) U_{t}^{-1} u \\
& =\left\{\sum_{j} \prod_{k} U_{t}\left(\chi_{j k} a_{j k}\left(z^{0} ; \cdot\right)\right) B_{j k}\left(z^{0}, z ; \theta^{0}\right)\right\} u,
\end{aligned}
$$

where $\left(U_{t} u\right)(x)=u\left(x+t \varphi_{z}\right)$. Also,

$$
\lim _{t \rightarrow+\infty}\left(U_{t} a_{j k}\left(z^{0} ; \cdot\right)\right)(x)=a_{j k}\left(z^{0}, z ; \cdot\right) .
$$


Therefore, instead of (2.33), we obtain

$$
\begin{aligned}
\inf \{ & \left.\left\|\sum_{j} \prod_{k} \chi_{j k} a_{j k}\left(z^{0} ; \cdot\right) B_{j k}\left(z^{0}, z ; \theta^{0}\right)+K ; \mathcal{B} L_{2}\left(\mathbb{R}^{n}\right)\right\| ; K \in \mathcal{J}_{z}\right\} \\
& =\left\|\sum_{j} \prod_{k} \chi_{j k}(z) a_{j k}\left(z^{0}, z ; \cdot\right) B_{j k}\left(z^{0}, z ; \theta^{0}\right) ; \mathcal{B} L_{2}\left(\mathbb{R}^{n}\right)\right\| .
\end{aligned}
$$

2.3.4. Localization of the algebra $\mathfrak{S}(z)$. If a point $z^{0}$ belongs to a zero-dimensional stratum $s_{0}$ of the partition $\mathcal{T}$ of $\mathcal{M}$, then localization of the initial algebra $\mathcal{A}$ leads to the algebra spanned by operators of the form $a\left(z^{0} ; x\right) F_{\xi \rightarrow x}^{-1} \Phi\left(z^{0} ; \xi\right) F_{y \rightarrow \xi}$; here $F$ is the Fourier transformation on $\mathbb{R}^{m}$. Such an algebra is isomorphic to the algebra $\mathfrak{S}\left(z^{0}\right)$ generated by the operator-valued functions

$$
\mathbb{R} \ni \lambda \rightarrow a\left(z^{0} ; \varphi\right) E_{\omega \rightarrow \varphi}(\lambda)^{-1} \Phi\left(z^{0} ; \omega\right) E_{\psi \rightarrow \omega}(\lambda) \in \mathcal{B} L_{2}\left(S^{m-1}\right) ;
$$

the norm on $\mathfrak{S}\left(z^{0}\right)$ is defined by $\left\|\mathfrak{B}(\cdot) ; \mathfrak{S}\left(z^{0}\right)\right\|=\sup \left\{\left\|\mathfrak{B}(\lambda) ; \mathcal{B} L_{2}\left(S^{m-1}\right)\right\| ; \lambda \in \mathbb{R}\right\}$ (see Proposition 2.11). If $z^{0} \in s_{0}$ and $\operatorname{dim} s_{0}=d_{0}>0$, then $\mathfrak{S}\left(z^{0}\right)$ arises under localization of the algebra $\mathcal{A}\left(z^{0}, \theta^{0}\right)$ at $z=0$. In more detail, first we arrive at the algebra $l_{0}$ of operators $a\left(z^{0} ; \cdot\right) A\left(z^{0} ; 0\right)$ (see item (ii) before Proposition 2.15), which is isomorphic to the algebra $\mathfrak{S}\left(z^{0}\right)$ spanned by the functions

$$
\mathbb{R} \ni \lambda \rightarrow a\left(z^{0} ; \varphi\right) E_{\omega \rightarrow \varphi}(\lambda)^{-1} \Phi\left(z^{0} ; \omega, 0\right) E_{\psi \rightarrow \omega}(\lambda) \in \mathcal{B} L_{2}\left(S^{n-1}\right),
$$

where $n=m-d_{0}$.

For applying the localization principle (Proposition $[2.8)$ to $\mathfrak{S}\left(z^{0}\right)$, we take $C_{0}(\mathbb{R}) \otimes$ $\mathcal{K} L_{2}\left(S^{n-1}\right)$ as the ideal $J$ and $C\left(S^{n-1}\right)$ as the localizing algebra $\mathcal{C}$ and verify that the hypotheses of Proposition 2.8 are fulfilled. Denote by $\mathfrak{S}_{\text {cont }}$ the subalgebra of $\mathfrak{S}\left(z^{0}\right)$ with continuous coefficients $a$. The inclusion $J=C_{0}(\mathbb{R}) \otimes \mathcal{K} L_{2}\left(S^{n-1}\right) \subset \mathfrak{S}_{\text {cont }}$ was proved in [11] (see also [10, Lemma 5.4.3]). Therefore, $J \subset \mathfrak{S}_{\text {cont }} \subset \mathfrak{S}\left(z^{0}\right)$. The operator of multiplication by a function $\alpha \in \mathcal{C}$ belongs to $\mathfrak{S}\left(z^{0}\right)$, i.e., $\mathcal{C} \subset \mathfrak{S}\left(z^{0}\right)$. Since the algebra $\mathcal{C}$ contains a unit, condition (i) of Proposition 2.8 is fulfilled. The sphere $S^{n-1}$ coincides with the maximal ideal space $\hat{\mathcal{C}}$ of the algebra $\mathcal{C}$. Suppose $\varphi_{1}, \varphi_{2} \in S^{n-1}$ and $\varphi_{1} \neq \varphi_{2}$. We choose $c_{1}$ and $c_{2}$ in $\mathcal{C}$ so that $c_{j}\left(\varphi_{j}\right)=1$ for $j=1,2$ and supp $c_{1} \cap \operatorname{supp} c_{2}=\varnothing$ and show that $c_{1} \mathfrak{S}\left(z^{0}\right) c_{2} \subset J$. It suffices to check that the commutator $[\mathfrak{A}, \alpha]$ is included in $J$ for any generator $\mathfrak{A}$ of the algebra $\mathfrak{S}\left(z^{0}\right)$ and any function $\alpha \in \mathcal{C}$. Therefore, a function of the form (2.37) (and also of the form (2.3) ) can be taken for the role of $\mathfrak{A}$. By 11, Proposition 2.2.1], the commutator $[\mathfrak{A}, \alpha]$ is a subset of $C_{0}(\mathbb{R}) \otimes \mathcal{K} L_{2}\left(S^{n-1}\right)$. Therefore, condition (ii) of Proposition 2.8 is also fulfilled. Finally, condition (iii) can easily be deduced from the fact that any irreducible representation of the ideal $J$ is equivalent to a certain representation $\pi(\lambda): \mathfrak{A} \mapsto \mathfrak{A}(\lambda)$. Thus, we have

$$
\widehat{\mathfrak{S}}\left(z^{0}\right)=\bigcup_{\varphi \in S^{n-1}} \widehat{\mathfrak{S}}\left(z^{0}\right)_{\varphi} \cup \widehat{J}
$$

where $\mathfrak{S}\left(z^{0}\right)_{\varphi}=\mathfrak{S}\left(z^{0}\right) / \mathcal{J}_{\varphi}$ is the local algebra at $\varphi$, and $\mathcal{J}_{\varphi}$ is the ideal of $\mathfrak{S}\left(z^{0}\right)$ spanned by the functions $\chi \in C\left(S^{n-1}\right)$ vanishing at $\varphi$.

For describing the local algebras $\mathfrak{S}\left(z^{0}\right) / \mathcal{J}_{\varphi}$, we use Proposition 2.16 below. Some preparations are required for its statement. Let $L^{\mathcal{N}}$ be a subalgebra of $L_{\infty}\left(S^{n-1}\right)$. We assume that $C\left(S^{n-1}\right) \subset L^{\mathcal{N}}$ and every element $a \in L^{\mathcal{N}}$ satisfies the following condition. Denote by $S_{\mathcal{N}}^{n-2}$ the unit sphere in the space tangent to $S^{n-1}$ at the north pole $\mathcal{N}=\left(0^{\prime}, 1\right)$ of $S^{n-1}$. Almost everywhere on $S_{\mathcal{N}}^{n-2}$ we have a limit

$$
\lim _{\varphi \rightarrow \mathcal{N}} a(\varphi)=a(\mathcal{N} ; \theta),
$$


which depends on the direction $\theta$ in which $\varphi$ approaches $\mathcal{N}$ and is uniform with respect to $\theta$. We introduce the algebra $\mathfrak{S}_{\mathcal{N}}$ generated by the operator-valued functions

$$
\mathbb{R} \ni \lambda \mapsto \mathfrak{B}(\lambda)=a(\varphi) E_{\omega \rightarrow \varphi}(\lambda)^{-1} \Phi(\omega) E_{\psi \rightarrow \omega}(\lambda),
$$

where $a \in L^{\mathcal{N}}$ and $\Phi \in C^{\infty}\left(S^{n-1}\right)$. The algebra $\mathfrak{S}_{\mathcal{N}}$ is equipped with the norm

$$
\left\|\mathfrak{B} ; \mathfrak{S}_{\mathcal{N}}\right\|=\sup \left\{\left\|\mathfrak{B}(\lambda) ; \mathcal{B} L_{2}\left(S^{n-1}\right)\right\| ; \lambda \in \mathbb{R}\right\} .
$$

Let $J(\mathcal{N})$ be the ideal in $\mathfrak{S}_{\mathcal{N}}$ spanned by the functions $\zeta$ Id with $\zeta \in C\left(S^{n-1}\right)$ and $\zeta(\mathcal{N})=0$. We denote by $\mathcal{L}$ the algebra generated by the functions

$$
\mathbb{R} \ni \lambda \mapsto a(\mathcal{N} ; \cdot) F_{\eta \rightarrow x^{\prime}}^{-1} \Phi(\eta, \lambda) F_{y^{\prime} \rightarrow \eta}: L_{2}\left(\mathbb{R}^{n-1}\right) \rightarrow L_{2}\left(\mathbb{R}^{n-1}\right),
$$

where $a(\mathcal{N} ; \cdot)$ is the function $\mathbb{R}^{n-1} \backslash 0 \ni x^{\prime} \mapsto a\left(\mathcal{N} ; x^{\prime} /\left|x^{\prime}\right|\right)$ and

$$
\|\mathfrak{D} ; \mathcal{L}\|=\sup \left\{\left\|\mathfrak{D}(\lambda) ; \mathcal{B} L_{2}\left(\mathbb{R}^{n-1}\right)\right\| ; \lambda \in \mathbb{R}\right\}
$$

for $\mathfrak{D} \in \mathcal{L}$.

Proposition 2.16. Let $\mathfrak{B}$ be an operator-valued function of the form (2.39). The mapping

$$
\mathfrak{B}(\lambda) \mapsto a(\mathcal{N} ; \cdot) F_{\eta \rightarrow x^{\prime}}^{-1} \Phi(\eta, \lambda) F_{y^{\prime} \rightarrow \eta}
$$

extends to an isomorphism $\mathfrak{S}_{\mathcal{N}} / J(\mathcal{N}) \simeq \mathcal{L}$.

This was proved in [9, Proposition 5.13]. We pass to describing the local algebras $\mathfrak{S}\left(z^{0}\right) / \mathcal{J}_{\varphi}$. Assume that $z^{0} \in s_{0}$, $\operatorname{dim} s_{0}=d_{0} \geq 0$, and $T(\mathcal{M})_{z^{0}}=T^{(m)}(\mathcal{M})_{z^{0}}$ is the tangent space of $\mathcal{M}$ at $z^{0}$. We denote by $T^{(n)}(\mathcal{M})_{z^{0}}$, where $n=m-d_{0}$, the subspace of $T^{(m)}(\mathcal{M})_{z^{0}}$ orthogonal to the stratum $s_{0}$. Choosing an arbitrary unit vector $z^{1}$ in $T^{(n)}(\mathcal{M})_{z^{0}}$, we introduce new orthogonal coordinates $\tilde{x}_{1}, \ldots, \tilde{x}_{n}$ in $T^{(n)}(\mathcal{M})_{z^{0}}$ in the same way as in a similar situation before. Namely, the axis $\tilde{x}_{n}, n=m-d_{0}$, is directed toward $z^{1}$; if $s_{1} \in \operatorname{st}\left(s_{0}\right), \operatorname{dim} s_{1}=s_{0}$, and $z^{1} \in \operatorname{pr}\left(s_{1}\right)$, then the axes $\tilde{x}_{m-d_{1}}, \ldots, \tilde{x}_{m-d_{0}-1}$ are parallel to the $\left(d_{1}-d_{0}-1\right)$-dimensional space tangent to the stratum $\operatorname{pr}\left(s_{1}\right)$ at $z^{1}$. The remaining axes $\tilde{x}_{1}, \ldots, \tilde{x}_{m-d_{1}-1}$ are orthogonal to $\operatorname{pr}\left(s_{1}\right)$ at $z^{1}$. We write the coordinate transformation in the form $x=J\left(z^{1}\right) \tilde{x}$ with the orthogonal matrix $J\left(z^{1}\right)$. In what follows we use the new coordinates, which will be denoted by $x_{1}, \ldots, x_{n}$. The algebra $\mathfrak{S}\left(z^{0}\right)$ is spanned by the operators of multiplication $a\left(z^{0} ; \cdot\right) I \in \mathcal{B} L_{2}\left(S^{n-1}\right)$ and the functions $\mathbb{R} \ni \lambda \mapsto \mathfrak{A}\left(z^{0} ; \lambda\right) \in \mathcal{B} L_{2}\left(S^{n-1}\right) ;$ here

$$
\mathfrak{A}\left(z^{0} ; \lambda\right)=E_{\omega \rightarrow \varphi}(\lambda)^{-1} \Phi\left(z^{0} ; J\left(z^{1}\right) \omega, 0\right) E_{\psi \rightarrow \omega}(\lambda),
$$

and 0 is the row $(0, \ldots, 0)$ containing $d_{0}$ elements (which is absent for $d_{0}=0$ ).

Denote by $\mathcal{L}\left(z^{0}, z^{1}\right)$ the algebra generated on $L_{2}\left(\mathbb{R}^{n-1}\right)$ by the operators $a\left(z^{0}, z^{1} ; \cdot\right) I$ and the functions of the form

$$
\mathbb{R} \ni \lambda \mapsto \mathfrak{D}\left(z^{0}, z^{1} ; \lambda\right)=F_{\eta \rightarrow x^{\prime}}^{-1} \Phi\left(z^{0} ; J\left(z^{1}\right)(\eta, \lambda), 0\right) F_{y^{\prime} \rightarrow \eta} \in \mathcal{B} L_{2}\left(\mathbb{R}^{n-1}\right) ;
$$

the norm in $\mathcal{L}\left(z^{0}, z^{1}\right)$ is introduced by (2.40). The next assertion follows from Proposition 2.16 .

Proposition 2.17. Let $\mathfrak{A}$ and $\mathfrak{D}$ be the same as in (2.41) and (2.42). Then the mapping

$$
a\left(z^{0} ; \cdot\right) \mapsto a\left(z^{0}, z^{1} ; \cdot\right), \quad \mathfrak{A}\left(z^{0} ; \cdot\right) \mapsto \mathfrak{D}\left(z^{0}, z^{1} ; \cdot\right)
$$

defined for generators of the algebra $\mathfrak{S}\left(z^{0}\right)$ extends up to an isomorphism

$$
\mathfrak{S}\left(z^{0}\right)_{z^{1}} \simeq \mathcal{L}\left(z^{0}, z^{1}\right)
$$

where $\mathfrak{S}\left(z^{0}\right)_{z^{1}}$ is the local algebra $\mathfrak{S}\left(z^{0}\right) / \mathcal{J}_{z^{1}}$ at $z^{1} \in S^{n-1}$. 
2.3.5. The dynasty of local algebras. In this section, with every (complete) chain of strata $s_{0}, \ldots, s_{k}$ of the partition $\mathcal{T}$ of $\mathcal{M}$ we associate the dynasty of $k+1$ generations of some local algebras. These algebras arise under iterations of the localization procedure. In the next section, any irreducible representation $\pi$ of $\mathcal{A}$ (up to equivalence) with $\operatorname{dim} \pi>1$ will be implemented in terms of such local algebras. The successive generations consist of algebras of the same type but more and more simple: the operators of the algebras act in the spaces $L_{2}\left(\mathbb{R}^{p}\right)$ or $L_{2}\left(S^{q}\right)$ with certain $p$ and $q$, and for a next generation the $p$ and $q$ reduce. The last generation of a dynasty is degenerate: localization of the algebras of the last generation provides only commutative algebras.

Under localization of $\mathcal{A}$ with the localizing algebra $C(\mathcal{M})$, only local algebras $\mathcal{A}_{z^{0}}$ of the following three types may arise:

1) If $z^{0} \in \mathcal{M} \backslash|\mathcal{T}|$, then $\mathcal{A}_{z^{0}} \simeq C\left(S^{*}(\mathcal{M})_{z^{0}}\right)$ (Proposition 2.9).

2) If $z^{0} \in s_{0}$, where $s_{0}$ is a stratum of $\mathcal{T}$ with $\operatorname{dim} s_{0}=d_{0}>0$, then, by Proposition 2.10 .

$$
\widehat{\mathcal{A}}_{z^{0}}=\bigcup_{\theta^{0} \in S^{d_{0}-1}} \widehat{\mathcal{A}}\left(z^{0} ; \theta^{0}\right) .
$$

The algebra $\mathcal{A}\left(z^{0} ; \theta^{0}\right)$ (for a fixed $\left.\theta^{0}\right)$ is generated on $L_{2}\left(\mathbb{R}^{m-d_{0}}\right)$ by operators of the form $a\left(z^{0} ; \cdot\right) A\left(z^{0} ; \theta^{0}\right)$ and is irreducible (see Proposition 2.12).

3) If $z^{0} \in s_{0}$ and $d_{0}=\operatorname{dim} s_{0}=0$, then $\mathcal{A}_{z^{0}} \simeq \mathfrak{S}\left(z^{0}\right)$ by Proposition 2.11, and $\mathfrak{S}\left(z^{0}\right)$ is spanned by the functions

$$
\mathbb{R} \ni \lambda \mapsto a\left(z^{0} ; \cdot\right) \mathfrak{A}\left(z^{0} ; \lambda\right) \in \mathcal{B} L_{2}\left(S^{m-1}\right) .
$$

Considering case 2), we use Proposition 2.15. The local algebras $\mathcal{A}\left(z^{0} ; \theta^{0}\right)_{z}$ in (i) and (iii) are commutative (see the items (i)-(v) before Proposition 2.15); we postpone for a while the discussion of (i) and (iii) and turn to items (ii), (iv), and (v). The algebra $\mathcal{A}\left(z^{0} ; \theta^{0}\right)_{z}$ for $z=0$ (item (ii)) is isomorphic to the algebra $\mathfrak{S}\left(z^{0}\right)$ of functions that range in $\mathcal{B} L_{2}\left(S^{n-1}\right)$, where $n=m-d_{0}$. In (iv), the local algebra $\mathcal{A}\left(z^{0} ; \theta^{0}\right)_{z}$ is isomorphic to the algebra of functions

$$
S^{d_{1}-d_{0}-1} \ni \theta^{1} \mapsto a\left(z^{0}, z ; \cdot\right) A\left(z^{0}, z ; 0, \theta^{1}\right),
$$

where

$$
A\left(z^{0}, z ; 0, \theta^{1}\right)=F_{\eta \rightarrow x^{(1)}}^{-1} \Phi\left(z^{0} ; J(z)\left(\eta, \theta^{1}\right), 0\right) F_{y^{(1)} \rightarrow \eta} \in \mathcal{B} L_{2}\left(\mathbb{R}^{m-d_{1}}\right) .
$$

We pass to item (v). Denote by $\hat{u}\left(y^{(1)}, \xi^{(2)}\right)=F_{y^{(2)} \rightarrow \xi^{(2)}} u\left(y^{(1)}, y^{(2)}\right)$ the partial Fourier transform of $u$. Then

$$
\left(B\left(z^{0}, z ; \theta^{0}\right) u\right)^{\wedge}\left(x^{(1)}, \xi^{(2)}\right)=F_{\xi^{(1)} \rightarrow x^{(1)}}^{-1} \Phi\left(z^{0} ; J(z)\left(\xi^{(1)}, \xi^{(2)}\right), \theta^{0}\right) F_{y^{(1)} \rightarrow \xi^{(1)}} \hat{u}\left(y^{(1)}, \xi^{(2)}\right) .
$$

We set $p=\left(\left|\xi^{(2)}\right|^{2}+1\right)^{1 / 2}, X=x^{(1)} p, Y=y^{(1)} p$, obtaining

$$
\begin{gathered}
\left(B\left(z^{0}, z ; \theta^{0}\right) u\right)^{\wedge}\left(X p^{-1}, \xi^{(2)}\right) \\
=(2 \pi)^{-\left(m-d_{0}\right)} \int_{\mathbb{R}^{m-d_{1}}} \exp \left(i X p^{-1} \xi^{(1)}\right) \Phi\left(z^{0} ; J(z)\left(\xi^{(1)}, \xi^{(2)}\right), \theta^{0}\right) d \xi^{(1)} \\
\times \int_{\mathbb{R}^{m-d_{1}}} \exp \left(-i \xi^{(1)} Y p^{-1}\right) \hat{u}\left(Y p^{-1}, \xi^{(2)}\right) p^{-1} d Y .
\end{gathered}
$$

We shall write $x, y\left(\in \mathbb{R}^{m-d_{1}}\right)$ instead of $X, Y$, and $v(y)$ and $w(x)$ instead of $u\left(Y p^{-1}, \xi^{(2)}\right)$ and $\left(B\left(z^{0}, z ; \theta^{0}\right) u\right)^{\wedge}\left(X p^{-1}, \xi^{(2)}\right)$. Then (2.46) takes the form

$$
w(x)=F_{\eta \rightarrow x}^{-1} \Phi\left(z^{0} ; J(z)\left(\eta, \xi^{(2)} / p\right), \theta^{0} / p\right) F_{y \rightarrow \eta} v(y) .
$$


Thus, the algebra $\mathcal{A}\left(z^{0}, \theta^{0}\right)_{z}$ is isomorphic to the algebra of functions

$$
\begin{aligned}
\mathbb{R}^{d_{1}-d_{0}} \ni \xi^{(2)} \mapsto & a\left(z^{0}, z ; x /|x|\right) \\
& \times F_{\eta \rightarrow x}^{-1} \Phi\left(z^{0} ; J(z)\left(\eta, \xi^{(2)} / p\right), \theta^{0} / p\right) F_{y \rightarrow \eta} v(y) \in \mathcal{B} L_{2}\left(\mathbb{R}^{m-d_{1}}\right) .
\end{aligned}
$$

Denote by $\mathcal{A}\left(z^{0}, z ; \theta^{0}, \theta^{1}\right)$ the algebra generated on $L_{2}\left(\mathbb{R}^{m-d_{1}}\right)$ by operators of the form

$$
a\left(z^{0}, z ; x /|x|\right) F_{\eta \rightarrow x}^{-1} \Phi\left(z^{0} ; J(z)\left(\eta, \theta^{1}\right), \theta^{0}\right) F_{y \rightarrow \eta}
$$

for fixed $\theta^{1} \in \mathbb{R}^{d_{1}-d_{0}}$ and $\theta^{0} \in \mathbb{R}^{d_{0}}$ with $\theta^{0} \neq 0$ and $\left|\theta^{0}\right|^{2}+\left|\theta^{1}\right|^{2}=1$. By (2.47),

$$
\widehat{\mathcal{A}}\left(z_{0} ; \theta_{0}\right)_{z}=\bigcup_{\theta^{1} \in \mathbb{R}^{d_{1}-d_{0}}} \widehat{\mathcal{A}}\left(z^{0}, z ; \theta^{0} /\left(1+\left|\theta^{1}\right|^{2}\right)^{1 / 2}, \theta^{1} /\left(1+\left|\theta^{1}\right|^{2}\right)^{1 / 2}\right) .
$$

We assume that $\mathcal{A}\left(z^{0} ; \theta^{0}\right)$ and $\mathfrak{S}\left(z^{0}\right)$ (for $d_{0} \geq 0$ ) form the first generation of local algebras. (The commutative algebras will be considered separately.)

We are going to form the second generation of local algebras. In that generation, first we include the algebras $\mathcal{A}\left(z^{0}, z^{1} ; \theta^{0}, \theta^{1}\right)$ introduced after formula (2.47) (we have changed the notation $z$ for $z^{1}$ ). In order to clarify the contribution of the algebras $\mathfrak{S}\left(z^{0}\right)$ to the second generation, we turn to Proposition 2.17 If $z^{1} \in S^{n-1} \backslash\left|\mathcal{T}\left(s_{0}\right)\right|$, $n=m-d_{0}$, then the algebra $\mathfrak{S}\left(z^{0}\right)_{z^{1}}$ is isomorphic to the algebra $C\left(S^{n-1}\right.$ ) (of all functions $S^{n-1} \ni \omega \mapsto \Phi\left(z^{0} ; J\left(z^{1}\right) \omega, 0\right)$; see (2.42) $)$. If $z^{1}$ belongs to a stratum $\operatorname{pr}\left(s_{1}\right)$ of the partition $\mathcal{T}\left(s_{0}\right)$, then the algebra $\mathfrak{S}\left(z^{0}\right)_{z^{1}}$ is isomorphic to the algebra of operatorvalued functions defined by (2.44) and (2.45) with $z=z^{1}$. Therefore, $\mathfrak{S}\left(z^{0}\right)$ in the second generation gives rise to the algebras $\mathcal{A}\left(z^{0}, z^{1} ; 0, \theta^{1}\right)$. Moreover, localization of $\mathcal{A}\left(z^{0}, z^{1} ; \theta^{0}, \theta^{1}\right)$ leads to the algebras $\mathfrak{S}\left(z^{0}, z^{1}\right)$ (as local ones at the origin) spanned by functions of the form

$$
\mathbb{R} \ni \lambda \mapsto a\left(z_{0}, z^{1} ; \varphi\right) E_{\omega \rightarrow \varphi}(\lambda)^{-1} \Phi\left(z^{0} ; J\left(z^{1}\right) \omega, 0\right) E_{\psi \rightarrow \omega}(\lambda) \in \mathcal{B} L_{2}\left(S^{m-d_{1}}\right) .
$$

The algebras $\mathfrak{S}\left(z^{0}, z^{1}\right)$ also must be included in the second generation.

Before listing the remaining generations, we simplify the notation. Let $s_{0}, \ldots, s_{k}$ be a chain of strata. Let us write the stratum $\mathrm{p}\left(s_{1}\right)$ of the partition $\mathcal{T}\left(s_{0}\right)$ in the form $s_{1}\left(s_{0}\right)$; by $s_{2}\left(s_{0}, s_{1}\right)$ we denote the stratum of $\mathcal{T}\left(s_{0}, s_{1}\right)$ generated by $s_{2}$; the symbols $s_{3}\left(s_{0}, \ldots, s_{2}\right), \ldots, s_{k}\left(s_{0}, \ldots, s_{k-1}\right)$ have a similar meaning.

Applying the above localization procedure to the second and the succeeding generations, with a chain $s_{0}, \ldots, s_{k}$ we associate a dynasty of $k+1$ generations, where the $j$ th generation consists of the algebras $\mathcal{A}\left(z^{0}, \ldots, z^{j-1} ; \theta^{0}, \ldots, \theta^{j-1}\right)$ and $\mathfrak{S}\left(z^{0}, \ldots, z^{j-1}\right)$ with $j \leq k+1$,

$$
\begin{gathered}
\mathcal{A}\left(z^{0}, \ldots, z^{j-1} ; \theta^{0}, \ldots, \theta^{j-1}\right) \subset L_{2}\left(\mathbb{R}^{m-d_{j-1}}\right), \quad z^{0} \in s_{0}, \ldots, z^{j-1} \in s_{j-1}\left(s_{0}, \ldots, s_{j-2}\right), \\
\theta^{j} \in \mathbb{R}^{q_{j}}, \quad\left|\theta^{0}\right|^{2}+\cdots+\left|\theta^{j-1}\right|^{2}=1, \quad q_{0}+\cdots+q_{j-1}=d_{j-1}=\operatorname{dim} s_{j-1}, \\
\mathfrak{S}\left(z^{0}, \ldots, z^{j-1}\right) \supset C_{0}(\mathbb{R}) \otimes \mathcal{K} L_{2}\left(S^{m-d_{j-1}-1}\right) .
\end{gathered}
$$

The algebra $\mathcal{A}\left(z^{0}, \ldots, z^{j-1} ; \theta^{0}, \ldots, \theta^{j-1}\right)$ is generated by the operators of multiplication $a\left(z^{0}, \ldots, z^{j-1} ; \cdot\right) I$ and the operators $A\left(z^{0}, \ldots, z^{j-1} ; \theta^{0}, \ldots, \theta^{j-1}\right)$ (similar types of operators appeared in (2.2) and (2.6) $)$. The algebra $\mathfrak{S}\left(z^{0}, \ldots, z^{j-1}\right)$ is spanned by the functions

$$
\mathbb{R} \ni \lambda \mapsto a\left(z^{0}, \ldots, z^{j-1} ; \cdot\right) \mathfrak{A}\left(z^{0}, \ldots, z^{j-1} ; \lambda\right) \in \mathcal{B} L_{2}\left(S^{m-d_{j-1}-1}\right) ;
$$

see (2.7). If the chain $s_{0}, \ldots, s_{k}$ is complete, then localization of the algebras in the $(k+1)$ st generation provides only commutative algebras. 
2.3.6. A list of irreducible representations $\pi$ of the algebra $\mathcal{A}$ with $\operatorname{dim} \pi>1$. We shall show that any irreducible representation $\pi$ of $\mathcal{A}$ such that $\operatorname{dim} \pi>1$ is equivalent either to one of the representations (2.8), (2.9) or to the identity representation. Recall that the identity representation of $\mathcal{A}$ is irreducible and $\mathcal{K} L_{2}(\mathcal{M}) \subset \mathcal{A}$. We start searching other representations with a localization of $\mathcal{A}$ (Proposition 2.9). All irreducible representations that are not equivalent to the identity representation also turn out to be representations of the quotient algebra $\mathcal{A} / \mathcal{K} L_{2}(\mathcal{M})$. The local algebras at the points $z \in \mathcal{M} \backslash|\mathcal{T}|$ are commutative; their irreducible representations are one-dimensional. Therefore, the representations $\pi \in \widehat{\mathcal{A}}$ with $\operatorname{dim} \pi>1$ can be found only under localization along chains of strata.

Let $s_{0}, \ldots, s_{k}$ be a complete chain of strata with $\operatorname{dim} s_{j}=d_{j}$. If $d_{0}>0$ and $z^{0} \in s_{0}$, then formula (2.43) is valid. The algebras $\mathcal{A}\left(z^{0} ; \theta^{0}\right)$ are irreducible. Hence, mappings of the form (2.8) for $j=0$ extend to irreducible representations $\pi\left(z^{0} ; \theta^{0}\right)$ of the algebra $\mathcal{A} / \mathcal{K} L_{2}(\mathcal{M})$. Such representations for distinct pairs $\left(z^{0}, \theta^{0}\right)$ are nonequivalent (they have distinct kernels). If $d_{0}=0$, then the local algebra $\mathcal{A}_{z^{0}}$ is isomorphic to the algebra $\mathfrak{S}\left(z^{0}\right)$ and we have (2.38). The representations (2.9) for $j=0$ extend to the representations $\pi\left(z^{0} ; \lambda_{0}\right)$ that arise in (2.38) as representations of the ideal $J$. These representations are irreducible and pairwise nonequivalent. If the partition $\mathcal{T}$ contains no zero-dimensional strata, then $\mathfrak{S}\left(z^{0}\right)$ arises under localization of $\mathcal{A}\left(z^{0} ; \theta^{0}\right)$, so that the representations (2.9) arise also for $j=0$.

In order to find representations of the local algebra $\mathfrak{S}\left(z^{0}\right)_{\varphi}$ in (2.38), we use Proposition 2.17. If $\varphi=z^{1} \in S^{n-1} \backslash\left|\mathcal{T}\left(s_{0}\right)\right|$, then the functions $\mathbb{R}^{n-1} \ni x \mapsto a\left(z^{0}, z^{1} ; x\right)$ turn out to be constant, $a\left(z^{0}, z^{1} ; x\right) \equiv a\left(z^{0} ; z^{1}\right)$. Therefore, the algebra $\mathfrak{S}\left(z^{0}\right) z^{1}$ is commutative and all its pairwise nonequivalent one-dimensional representations are

$$
\begin{aligned}
& \pi\left(z^{0} ; \varphi, \omega\right): a\left(z^{0} ; \cdot\right) \mapsto a\left(z^{0} ; \varphi\right), \\
& \pi\left(z^{0} ; \varphi, \omega\right): \mathfrak{A}\left(z^{0} ; \cdot\right) \mapsto \Phi\left(z^{0} ; J(\varphi) \omega, 0\right),
\end{aligned}
$$

where $\mathfrak{A}\left(z^{0} ; \cdot\right)$ is a function of the form (2.41), $\varphi=z^{1} \in S^{n-1} \backslash\left|\mathcal{T}\left(s_{0}\right)\right|$, and $\omega$ runs over $S^{n-1}$; note that the directions of the vectors $\omega=\left(0, \ldots, 0, \omega_{n}\right)$ and $\varphi$ coincide. If $z^{1} \in s_{1} \in \mathcal{T}\left(s_{0}\right), d_{1}=\operatorname{dim} s_{1}$, then the same argument as in the proof of Proposition 2.10 can be employed to verify that the algebra $\mathfrak{S}\left(z^{0}\right) z^{1}$ is isomorphic to the algebra of functions

$$
\begin{aligned}
S^{d_{1}-1} \ni \theta^{1} & \mapsto A\left(z^{0}, z^{1} ; 0, \theta^{1}\right) \\
& =a\left(z^{0}, z^{1} ; x\right) F_{\eta \rightarrow x}^{-1} \Phi\left(z^{0} ; J\left(z^{1}\right)\left(\eta, \theta^{1}\right), 0\right) F_{y \rightarrow \eta} \in \mathcal{B} L_{2}\left(\mathbb{R}^{m-d_{1}}\right),
\end{aligned}
$$

with the norm $\sup \left\{\left\|A\left(z^{0}, z^{1} ; 0, \theta^{1}\right) ; \mathcal{B} L_{2}\left(\mathbb{R}^{m-d_{1}}\right)\right\| ; \theta^{1} \in S^{d_{1}-1}\right\}$. Therefore,

$$
\left(\mathfrak{S}\left(z^{0}\right) z^{1}\right)^{\wedge}=\bigcup_{\theta^{1} \in S^{d_{1}-1}} \mathcal{A}\left(z^{0}, z^{1} ; 0, \theta^{1}\right)^{\wedge}
$$

(compare with (2.48) ). Thus, to obtain further information on representations of $\mathfrak{S}\left(z^{0}\right) z^{1}$ we need to deal with algebras $\mathcal{A}\left(z^{0}, z^{1} ; 0, \theta^{1}\right)$ of the second generation.

The description of localization for the algebras $\mathcal{A}\left(z^{0} ; \theta^{0}\right)$ and $\mathfrak{S}\left(z^{0}\right)$ shows that new irreducible representations of dimension exceeding 1 can occur only in the spectrum of local algebras of succeeding generations. The same argument as applied to the algebras $\mathcal{A}\left(z^{0}, z^{1} ; \theta^{0}, \theta^{1}\right)$ and $\mathfrak{S}\left(z^{0}, z^{1}\right)$ yields the representations $\pi\left(z^{0}, z^{1} ; \theta^{0}, \theta^{1}\right)$ and $\pi\left(z^{0}, z^{1} ; \lambda_{1}\right)$. Going from a generation to the succeeding generation in a dynasty of local algebras, we find all representations (2.8) and (2.9). In accordance with the localization principle, such a list contains all irreducible representations of the algebra $\mathcal{A}$ of dimension exceeding 1 (up to equivalence). 
2.3.7. The one-dimensional representations of the algebra $\mathcal{A}$. We list all one-dimensional representations arising at various stages of the localization procedure. Assume that $x \in \mathcal{M} \backslash|\mathcal{T}|$. The local algebra $\mathcal{A}_{x}$ is commutative; any representation of $\mathcal{A}_{x}$ is included in (2.10); in that case $\mathrm{p}(x)=x$ and the coefficients $a$ are continuous at the point $x$.

Suppose that $s_{0}$ is a stratum of the partition $\mathcal{T}, d_{0}=\operatorname{dim} s_{0}>0$, and $z^{0} \in s_{0}$. Let $T\left(z^{0}, s_{0}\right)\left(T^{*}\left(z^{0}, s_{0}\right)\right)$ be the tangent (cotangent) space to $\mathcal{M}$ orthogonal to $s_{0}$. Also suppose $z \in T\left(z^{0}, s_{0}\right), z \neq 0$, and $\varphi=z /|z| \notin\left|\mathcal{T}\left(s_{0}\right)\right|$. Finally, let $\omega$ be a unit vector in $T^{*}\left(z^{0}, s_{0}\right)$. By Proposition 2.15] and formulas (2.26), the mapping

$$
\begin{aligned}
& \pi\left(z^{0} ; \varphi, \omega, 0\right): a\left(z^{0} ; \cdot\right) \mapsto a\left(z^{0} ; \varphi\right), \\
& \pi\left(z^{0} ; \varphi, \omega, 0\right): A\left(z^{0} ; \theta^{0}\right) \mapsto \Phi\left(z^{0} ; \omega, 0\right)
\end{aligned}
$$

determines a one-dimensional representation of the algebra $\mathcal{A}\left(z^{0} ; \theta^{0}\right)$.

Suppose that $z$ is a point in the infinitely distant sphere of $T\left(z^{0}, s_{0}\right), \varphi$ is the unit vector directed toward $z$, and $\varphi \notin\left|\mathcal{T}\left(s_{0}\right)\right|$. Then, by (2.28), the mapping

$$
\begin{gathered}
a\left(z^{0} ; \cdot\right) \mapsto a\left(z^{0} ; \varphi\right), \\
A\left(z^{0} ; \theta^{0}\right) \mapsto \Phi\left(z^{0} ; \eta, \theta^{0}\right)
\end{gathered}
$$

gives rise to a one-dimensional representation of $\mathcal{A}\left(z^{0} ; \theta^{0}\right)$ for any $\eta \in T^{*}\left(z^{0}, s_{0}\right)$.

Since the function $\xi \mapsto \Phi\left(z^{0} ; \xi\right)$ is homogeneous, we can combine (2.51) and (2.52) to show that the mapping

$$
\begin{aligned}
& \pi\left(z^{0} ; \varphi, \xi\right): a\left(z^{0} ; \cdot\right) \mapsto a\left(z^{0} ; \varphi\right) \\
& \pi\left(z^{0} ; \varphi, \xi\right): A\left(z^{0} ; \theta^{0}\right) \mapsto \Phi\left(z^{0} ; \xi\right)
\end{aligned}
$$

determines a one-dimensional representation of $\mathcal{A}\left(z^{0} ; \theta^{0}\right)$ for any $\xi \in S^{*}(\mathcal{M})_{z^{0}}$ and every unit vector $\varphi \in T\left(z^{0}, s_{0}\right)$. The representations of the form (2.53) are among those indicated in (2.10). Note that the $\pi\left(z^{0} ; \varphi, \omega, 0\right)$ in (2.51) also turn out to be representations of the algebra $\mathfrak{S}\left(z^{0}\right)$; see (2.49). If $d_{0}=\operatorname{dim} s_{0}=0$, then the representations (2.52) are absent and formulas (2.51) and (2.53) coincide and determine representations of $\mathfrak{S}\left(z^{0}\right)$.

In essence, the listing of the one-dimensional representations under further localization along chains of strata reduces to the argument given above. As a result, we get all representations in (2.10). The localization principle implies that there are no other onedimensional representations of $\mathcal{A}$.

\section{§3. A solving COmposition series in the Algebra $\mathcal{A}$}

3.1. Preliminaries. In this section, first we recall some information on continuous fields of algebras. Then we discuss a relationship between the structure of solving composition series and the spectral topology of an arbitrary solvable algebra. Our idea of constructing a solving series is explained by examples. In Subsection 3.2, this idea will be implemented for the algebra $\mathcal{A}$ discussed in $\S 2$; the examples mentioned above will be used in Subsection 3.2 and in the proof of the main theorem. At the end of Subsection 3.1. we formulate a theorem that describes the spectral topology of the algebra $\widehat{\mathcal{A}}$.

3.1.1. Continuous fields of $C^{*}$-algebras. We denote by $\mathcal{B}\left(E, E^{\prime}\right)$ the space of linear operators acting from a Banach space $E$ to a Banach space $E^{\prime}$ (if $E=E^{\prime}$, we write $\mathcal{B}(E)$ ). Let $U(H)$ denote the group of unitary operators on a Hilbert space $H$. Also, we introduce the space $C(T, L)$ of continuous functions on $T$ that range in $L$, where $T$ and $L$ are topological spaces. 
Suppose that a Banach space $E_{t}$ corresponds to any point $t \in T$. By definition, a vector field on $T$ is any mapping

$$
x: T \rightarrow \prod_{t \in T} E_{t},
$$

subject to the condition $x(t) \in E_{t}$ for all $t \in T$. Sometimes it is convenient to identify a vector field $x$ and its image $\{x(t)\} \subset \prod E_{t}$.

A continuous field $\mathcal{E}$ of Banach spaces on $T$ is a family of Banach spaces $\left\{E_{t}\right\}_{t \in T}$ equipped with a set $\Gamma$ of vector fields such that

(i) $\Gamma$ is a linear space;

(ii) the set $\{x(t): x \in \Gamma\}$ is dense in $E_{t}$ for every $t \in T$;

(iii) the function $t \mapsto\|x(t)\|$ is continuous for any $x \in \Gamma$;

(iv) if $y \in \prod_{t \in T} E_{t}$ is a vector field and for any $t_{0} \in T$ and $\varepsilon>0$ there exists a field $x \in \Gamma$ for which $\|y(t)-x(t)\|<\varepsilon$ in a neighborhood of $t_{0}$, then $y \in \Gamma$.

The elements $x \in \Gamma$ are called continuous vector fields in $\mathcal{E}$. The space $\Gamma$ is a module over the ring $C(T)$ of complex functions on $T$ (see [18, 10.1.9]).

An isomorphism of continuous fields $\mathcal{E}=\left(\left\{E_{t}\right\}_{t \in T}, \Gamma\right)$ and $\mathcal{E}^{\prime}=\left(\left\{E_{t}^{\prime}\right\}_{t \in T}, \Gamma^{\prime}\right)$ is a family $\varphi=\{\varphi(t)\}_{t \in T}$ of linear operators $\varphi(t): E_{t} \rightarrow E_{t}^{\prime}$ such that

1) for every $t$ the operator $\varphi(t)$ is an isometric isomorphism of $E_{t}$ on $E_{t}^{\prime}$;

2) $\varphi(\Gamma) \subset \Gamma^{\prime}$ (which is equivalent to $\left.\varphi(\Gamma)=\Gamma^{\prime}\right)$.

Example. Consider the product $T \times E$, where $E$ is a Banach space. We assume that $E_{t}=\{t\} \times E, q: T \times E \rightarrow E$ is a projection, and $q_{t}=q \mid E_{t}$. The mapping $q$ enables us to endow every fiber $E_{t}$ with a Banach space structure so that the operators $q_{t}: E_{t} \rightarrow E$ become isometric isomorphisms. As $\Gamma$ we take the set of all vector fields $t \mapsto(t, f(t))$, where $f \in C(T, E)$. Then $\left(\left\{E_{t}\right\}, \Gamma\right)$ is a continuous field of Banach spaces, which is called a constant field. We denote it (somewhat loosely) by $T \times E$ and write $\Gamma(T \times E)$ instead of $\Gamma$. A field isomorphic to a constant field is said to be trivial.

Let $\mathcal{E}=\left(\left\{E_{t}\right\}_{t \in T}, \Gamma\right)$ be a continuous field of Banach spaces on $T$ and $\Omega \subset T$ an open set. Denote by $\Gamma_{\Omega}$ the set of vector fields on $\Omega$ that are limits of elements in $\Gamma \mid \Omega$ with respect to the local uniform convergence. Then $\left(\left\{E_{t}\right\}_{t \in \Omega}, \Gamma_{\Omega}\right)$ is a continuous field of Banach spaces on $\Omega$, denoted by $\mathcal{E} \mid \Omega$; the field $\mathcal{E} \mid \Omega$ is said to be induced by $\mathcal{E}$.

A field $\mathcal{E}$ is locally trivial if any point $t$ has a neighborhood $\Omega$ such that the field $\mathcal{E} \mid \Omega$ is trivial. Let $\mathcal{E}$ be a locally trivial field of Banach spaces on $T$ and $\left\{\Omega_{i}\right\}_{i \in I}$ an open covering of $T$ such that all the fields $\mathcal{E} \mid \Omega_{i}$ are trivial and $\varphi_{i}: T \times E_{i} \rightarrow \mathcal{E} \mid \Omega_{i}$ are isomorphisms of trivial fields. The family of charts $\left\{\left(\varphi_{i}, \Omega_{i}\right)\right\}_{i \in I}$ is called a trivializing atlas (or simply atlas) for $\mathcal{E}$.

Let $\mathcal{E}=\left(\left\{E_{t}\right\}_{t \in T}, \Gamma\right)$ be a continuous field of Banach spaces. A set $\Lambda \subset \Gamma$ is said to be total if for every $t \in T$ the set $\{x(t): x \in \Lambda\}$ is total in $E_{t}$ (i.e., the closure of its linear hull coincides with $E_{t}$ ). A field is separable if $\Gamma$ contains a countable total subset.

Lemma 3.1 ([19, 10.2.7]). Let $T$ be a separable metrizable space and $\mathcal{E}=\left(\left\{E_{t}\right\}, \Gamma\right\} a$ locally trivial continuous field of Banach spaces on $T$. If every $E_{t}$ is separable, then $\mathcal{E}$ is also separable.

Lemma 3.2 (19, 10.8.7]). Let $T$ be a finite-dimensional (in the sense of topological dimension) paracompact space and $\mathcal{E}=\left(\left\{E_{t}\right\}_{t \in T}, \Gamma\right)$ a separable continuous field of Hilbert spaces. Suppose that the dimension of each $E_{t}$ is $\aleph_{0}$. Then the field $\mathcal{E}$ is trivial.

Suppose that $\varphi: T \times E \rightarrow T \times E^{\prime}$ is an isomorphism of trivial fields. Then $\varphi(t, e)=$ $\left(t, A_{t} e\right)$ for every $t \in T$, where $A_{t}$ is a linear isometry $E \rightarrow E^{\prime}$ and $A_{t}=q_{t}^{\prime} \varphi(t) q_{t}^{-1}$. 
Lemma 3.3. Let $\varphi: T \times E \rightarrow T \times E^{\prime}$ be an isomorphism of trivial fields, and let $A_{t}=q_{t}^{\prime} \varphi(t) q_{t}^{-1}$ for $t \in T$. Then the function $t \mapsto A_{t} \in \mathcal{B}\left(E, E^{\prime}\right)$ is continuous in the strong operator topology.

Proof. We fix $e \in E$. Since the vector field $t \mapsto(t, e)$ belongs to $\Gamma(T \times E)$, its image $t \mapsto\left(t, A_{t} e\right)$ under $\varphi$ is in $\Gamma\left(T \times E^{\prime}\right)$. Hence, the function $t \mapsto A_{t} e$ is continuous.

By definition, a continuous field of $C^{*}$-algebras on $T$ is a continuous field $\left(\left\{\mathcal{A}_{t}\right\}, \Theta\right)$ of Banach spaces where every space $\mathcal{A}_{t}$ is endowed with multiplication and involution that convert $\mathcal{A}_{t}$ into a $C^{*}$-algebra and leave $\Theta$ invariant. An isomorphism of fields $\left(\left\{\mathcal{A}_{t}\right\}, \Theta\right)$ and $\left(\left\{\mathcal{A}_{t}^{\prime}\right\}, \Theta^{\prime}\right)$ is given by a family $\{\varphi(t)\}_{t \in T}$, where $\varphi(t): \mathcal{A}_{t} \rightarrow \mathcal{A}_{t}^{\prime}$ is an isomorphism for each $t$ and $\varphi(\Theta) \subset \Theta^{\prime}$. The notions of triviality and local triviality of fields of $C^{*}$-algebras are introduced in accordance with this definition.

Let $\left(\left\{A_{t}\right\}, \Theta\right)$ be a continuous field of $C^{*}$-algebras for which all the $A_{t}$ are elementary (i.e., every algebra $A_{t}$ is isomorphic to the ideal of compact operators on a Hilbert space). Then $\left(\left\{A_{t}\right\}, \Theta\right)$ is called a field of elementary $C^{*}$-algebras.

Suppose that $T$ is a locally compact space and $\mathcal{L}=\left(\left\{A_{t}\right\}, \Theta\right)$ is a continuous field of $C^{*}$-algebras on $T$. We denote by $\mathcal{A}$ the set of vector fields $x \in \Theta$ such that $\|x(t)\|$ tends to zero as $t \rightarrow \infty$. Then $\mathcal{A}$ is an involutive subalgebra of $\Theta$. For $x \in \mathcal{A}$, we set $\|x\|=\sup \{\|x(t)\| ; t \in T\}$. Equipped with this norm, $\mathcal{A}$ becomes a $C^{*}$-algebra, which is called the $C^{*}$-algebra determined by the field $\mathcal{L}$. For instance, if $\mathcal{L}$ is a trivial field, then $\mathcal{A} \cong C_{0}(T, \mathcal{B}) \cong C_{0}(T) \otimes \mathcal{B}$, where $\mathcal{B}$ is some $C^{*}$-algebra. If $\mathcal{L}$ is a trivial field of elementary algebras, we have $\mathcal{A} \simeq C_{0}(T) \otimes \mathcal{K} H$.

Proposition $3.4([19,10.4 .3])$. Assume that $T$ is a locally compact space, $\mathcal{L}=\left(\left\{\mathcal{A}_{t}\right\}, \Theta\right)$ is a continuous field of algebras on $T$, and $\mathcal{A}$ is the algebra determined by $\mathcal{L}$. For $t \in T$ and $\pi \in \widehat{\mathcal{A}}_{t}$, we denote by $\rho_{\pi}$ the irreducible representation $x \mapsto \pi(x(t))$ of $\mathcal{A}$. Then $\pi \mapsto \rho_{\pi}$ is a bijection of the union of the sets $\widehat{\mathcal{A}}_{t}$ onto $\widehat{\mathcal{A}}$.

The next proposition contains a description of the topology on the spectrum $\widehat{\mathcal{A}}$ for the case where the field $\mathcal{L}$ is trivial.

Proposition 3.5 ([19, 10.10.2]). Assume that $T$ is a locally compact space, $\mathcal{B}$ is a $C^{*}$-algebra, and $\mathcal{A}$ is the $C^{*}$-algebra of continuous mappings from $T$ into $\mathcal{B}$ tending to zero at infinity. Then the space $\widehat{\mathcal{A}}$ can be canonically identified with $T \times \widehat{\mathcal{B}}$.

3.1.2. Sufficient triviality condition for fields of elementary algebras. Let $T$ be a finitedimensional paracompact space and $H$ a Hilbert space with $\operatorname{dim} H=\aleph_{0}$. When considering the trivial fields $\Omega \times H$ and $\Omega \times \mathcal{K} H$, where $\Omega$ is an open subset of $T$, we shall denote the "fibers" $\{t\} \times H$ and $\{t\} \times \mathcal{K} H$ by $H_{t}$ and $(\mathcal{K} H)_{t}$.

Assume that $\mathcal{E}=\left(\left\{E_{t}\right\}_{t \in T}, \Gamma\right)$ is a separable continuous field of Hilbert spaces, $\operatorname{dim} E_{t}=\aleph_{0}$, and $\mathcal{L}=\left(\left\{\mathcal{K} E_{t}\right\}_{t \in T}, \Theta\right)$ is a continuous field of elementary $C^{*}$-algebras (for the uniformity of notation, in what follows we write $\Gamma(\mathcal{L})$ instead of $\Theta$ ). By Lemma 3.2 there exists an isomorphism $\varphi: T \times H \rightarrow \mathcal{E}$. It is easy to check that if for any $K(\cdot) \in \Gamma(\mathcal{L})$ the vector field $t \mapsto \varphi^{-1}(t) K(t) \varphi(t)$ belongs to $\Gamma(T \times \mathcal{K} H)$, then the field $\mathcal{L}$ is trivial. This simple condition ensuring the triviality of $\mathcal{L}$ is not efficient in specific situations because of difficulties associated with constructing $\varphi$ in an explicit form. The next Proposition 3.6 (taken from [8]) provides a sufficient triviality condition for $\mathcal{L}$ in terms of local trivialization of the field $\mathcal{E}$.

Proposition 3.6. Let $T, H, \mathcal{E}$, and $\mathcal{L}$ be the same as above, and let $\left\{\left(\varphi_{i}, \Omega_{i}\right)\right\}_{i \in I}$ be an atlas for $\mathcal{E}$. Suppose that for all $i \in I$ and $K \in \Gamma(\mathcal{L})$ the vector field $t \mapsto \varphi_{i}(t)^{-1} K(t) \varphi_{i}(t)$ on $\Omega_{i}$ belongs to $\Gamma\left(\Omega_{i} \times \mathcal{K} H\right)$. Then the field $\mathcal{L}$ is trivial, and consequently, the $C^{*}$-algebra determined by this field is isomorphic to $C_{0}(T) \otimes \mathcal{K} H$. 
We preface the proof with the following lemma, where $U(H)$ stands for the unitary group of the Hilbert space $H$.

Lemma 3.7. Let $t \mapsto R(t) \in \mathcal{K} H$ and $t \mapsto u(t) \in U(H)$ be operator-valued functions on an open set $\Omega \subset T$ while $R$ is continuous in the uniform topology and $u$ is continuous in the strong operator topology. Then the function $t \mapsto u(t) R(t) u(t)^{-1}$ is continuous in the uniform topology.

Proof of the lemma. The continuity of $u R$ (relative to the uniform topology) is evident. Then the function $u R u^{-1}=\left(u(u R)^{*}\right)^{*}$ is also continuous.

Proof of Proposition 3.6. Let $\varphi=\{\varphi(t)\}_{t \in T}$ be an isomorphism $T \times H \rightarrow \mathcal{E}$. For every vector field $K(\cdot) \in \Gamma(\mathcal{L})$ we define a field

$$
T \ni t \mapsto\left(t, q_{t} \varphi(t)^{-1} K(t) \varphi(t) q_{t}^{-1}\right) \in(\mathcal{K} H)_{t},
$$

where $q_{t}$ is the restriction of the projection $q: T \times H \rightarrow H$ to the "fiber" $H_{t}$. The proposition will be proved if we check the continuity (in the uniform topology) of the function

For this, note that

$$
t \mapsto Q(t):=q_{t} \varphi(t)^{-1} K(t) \varphi(t) q_{t}^{-1} .
$$

$$
\left(Q \mid \Omega_{i}\right)(t)=u_{i}(t) R_{i}(t) u_{i}(t)^{-1}
$$

for any $i \in I$, where $R_{i}(t)=q_{t} \varphi_{i}(t)^{-1} K(t) \varphi_{i}(t) q_{t}^{-1}$ and $u_{i}(t)=q_{t}\left(\varphi^{-1} \circ \varphi_{i}\right) q_{t}^{-1}$. The hypotheses of the proposition imply that $R_{i}$ belongs to $C\left(\Omega_{i}, \mathcal{K} H\right)$. Moreover, the function $u_{i}$ is continuous in the strong operator topology, which follows from Lemma 3.3 because $\varphi^{-1} \circ \varphi_{i}$ is an isomorphism of the fields $\Omega_{i} \times H$ and $(T \times H) \mid \Omega_{i}$. Applying Lemma 3.7 and using (3.1), we see that the function $Q \mid \Omega_{i}$ is continuous in the uniform topology. Then $Q \in C(T, \mathcal{K} H)$ because $i \in I$ is arbitrary.

3.1.3. Relationship between the solvability of an algebra and the topology of the spectrum. Let $\mathcal{D}=C_{0}(X) \otimes \mathcal{K} H \simeq C_{0}(X, \mathcal{K} H)$, where $X$ is a locally compact Hausdorff space. Any nonzero irreducible representation of $\mathcal{D}$ is a mapping of the form $\mathcal{D} \ni f \mapsto f(x) \in \mathcal{K} H$ for a point $x \in X$. The Jacobson topology on the spectrum $\widehat{\mathcal{D}}$ coincides with the topology of $X$. Therefore, $\widehat{\mathcal{D}}$ can be identified with $X$ (Propositions 3.4 and 3.5). Suppose that $\mathcal{L}$ is a solvable algebra (see Definition 1.1) and $\{0\}=I_{-1} \subset I_{0} \subset \cdots \subset I_{N}=\mathcal{L}$ is a solving composition series, while

$$
I_{j} / I_{j-1} \simeq C_{0}\left(X_{j}\right) \otimes \mathcal{K} H_{j}, \quad j=0, \ldots, N<\infty .
$$

Then $\left(I_{j} / I_{j-1}\right)^{\wedge}=X_{j}$, whence $\widehat{I}_{j}=G_{j}$, where $G_{j}=\bigcup_{i=0}^{j} X_{i}$. Being the spectrum of an ideal, $G_{j}$ is an open subset of the spectrum $\widehat{\mathcal{L}}$ of $\mathcal{L}$.

Now we dwell on the definition of a solvable algebra. Let $\mathcal{A}$ be an arbitrary $C^{*}$-algebra. Every element $a \in \mathcal{A}$ gives rise to a function $\widehat{a}$ given on the spectrum $\widehat{\mathcal{A}}$ by the formula $\widehat{a}(\pi)=\pi a\left(\in \mathcal{B} H_{\pi}\right)$. The functions $\widehat{a}$ form an algebra isomorphic to $\mathcal{A}$, because

$$
\|\widehat{a}\|=\sup \left\{\left\|\widehat{a}(\pi) ; \mathcal{B} H_{\pi}\right\| ; \pi \in \widehat{\mathcal{A}}\right\}=\|a\|
$$

(see [19, 2.7.3]). In general, the field of $C^{*}$-algebras $\left(\{\pi(\mathcal{A})\}_{\pi \in \hat{\mathcal{A}}}, \Theta\right)$ may fail to be continuous for any choice of the set $\Theta \subset \prod_{\pi \in \hat{\mathcal{A}}} \mathcal{B} H_{\pi}$ of vector fields on $\widehat{\mathcal{A}}$ containing $\{\widehat{a}: a \in \mathcal{A}\}$ because, as a rule, the space $\widehat{\mathcal{A}}$ endowed with the Jacobson topology is not Hausdorff, so that the functions $\pi \mapsto\left\|\widehat{a}(\pi) ; \mathcal{B} H_{\pi}\right\|$ are not continuous (only lower semicontinuity can be guaranteed; see [19, 3.3.2]). Assume that there exist open subsets $G_{k}$ of the spectrum $\widehat{\mathcal{A}}$ such that

$$
\varnothing=G_{-1} \subset G_{0} \subset \cdots \subset G_{N}=\widehat{\mathcal{A}}
$$


where $X_{k}:=G_{k} \backslash G_{k-1}$ is a locally compact Hausdorff space for all $k \in\{0, \ldots, N\}$. With every $G_{k}$ we associate the ideal

$$
I_{k}=\left\{a \in \mathcal{A}: \widehat{a}(\pi)=0 \text { for } \pi \in \widehat{\mathcal{A}} \backslash G_{k}\right\} .
$$

The spectrum of this ideal can be identified with $G_{k}$, and the spectrum of $I_{k} / I_{k-1}$ coincides with $X_{k}$. Any element $a$ in $I_{k}$ gives rise to a vector field $\widehat{a}$ on $X_{k}$, which depends only on the residue class $a+I_{k-1}$. Let $\Theta_{k}$ be the local closure of the set of such fields. Then $\left(\left\{\pi\left(I_{k}\right)\right\}_{\pi \in X_{k}}, \Theta_{k}\right)$ is a continuous field of $C^{*}$-algebras (continuity follows from the separability of $X_{k}$; see [19, 3.3.9]).

The solvability of $\mathcal{A}$ means that the sets $G_{k}$ satisfying the above conditions can be chosen for all $k$ in such a way that

(i) $\pi\left(I_{k}\right)=\mathcal{K} H_{\pi}$ for $\pi \in X_{k}$;

(ii) the field of the algebras $\mathcal{L}_{k}:=\left(\left\{\mathcal{K} H_{\pi}\right\}_{\pi \in X_{k}}, \Theta_{k}\right)$ is trivial.

In this case the algebra $\mathcal{A}_{k}$ determined by the field $\mathcal{L}_{k}$ is isomorphic to $C_{0}\left(T_{k}\right) \otimes \mathcal{K} H$. On the other hand, since $\mathcal{A}_{k} \cong I_{k} / I_{k-1}, k=0, \ldots, N$ (see [19, 10.5.4]), for every $k$ we have an isomorphism $I_{k} / I_{k-1} \simeq C_{0}\left(X_{k}\right) \otimes \mathcal{K} H_{k}$.

The following examples serve, in particular, to illustrate a rather cumbersome description (in Subsection 3.1.4) of the Jacobson topology ( $J$-topology) on the spectrum of the algebra $\mathcal{A}$ generated by pseudodifferential operators with discontinuous coefficients (i.e., the algebra treated in $\S 2$ ). Moreover, these examples will be used for the verification of the properties of solving composition series in $\mathcal{A}$. Recall (see, e.g., [19]) that a base of the $J$-topology on the spectrum $\widehat{\mathcal{A}}$ is formed by the sets $\{\pi \in \widehat{\mathcal{A}}:\|\pi a\|>1\}$, where $a$ runs over a dense set in $\mathcal{A}$. However, in what follows there are no immediate references to that definition of $J$-topology; we simply indicate fundamental systems of neighborhoods for the points in $\widehat{\mathcal{A}}$.

Example 3.8. The algebra $\mathcal{A}$ is generated on $L_{2}(\mathcal{M})$ by pseudodifferential operators in $\Psi^{0}(\mathcal{M})$ (with smooth symbols). The spectrum $\widehat{\mathcal{A}}$ is parametrized by the points of the disjoint union $\Xi=S^{*}(\mathcal{M}) \sqcup e$; the point $e$ corresponds to the identity representation, $(x, \xi) \in S^{*}(\mathcal{M})_{x}$ is related to the one-dimensional representation $\pi(x, \xi): A \mapsto \Phi(x, \xi)$, where $\Phi$ is the principal symbol of $A$. The point $e$ is an open set, and its closure $\bar{e}$ coincides with $\Xi$. Any neighborhood of a point $(x, \xi)$ in the $J$-topology is $\mathcal{U} \cup e$, where $\mathcal{U}$ is a usual neighborhood of $(x, \xi)$ in $S^{*}(\mathcal{M})$. The filtration (3.2) is of the form $\varnothing \subset e \subset \Xi=\widehat{\mathcal{A}}$. In accordance with (3.3), we set $I_{0}=\{a \in \mathcal{A}: \pi(x, \xi) a=0$ for all $\left.(x, \xi) \in S^{*}(\mathcal{M})\right\}$ and $I_{1}=\mathcal{A}$, obtaining the series $0 \subset \mathcal{K} L_{2}(\mathcal{M}) \subset \mathcal{A}$. Clearly, $I_{1} / I_{0}=\mathcal{A} / \mathcal{K} L_{2}(\mathcal{M}) \simeq C\left(S^{*}(\mathcal{M})\right)$.

Example 3.9. The algebra $\mathfrak{S}$ is spanned by functions of the form

$$
\mathbb{R} \ni \lambda \mapsto \mathfrak{B}(\lambda)=a(\varphi) E_{\omega \rightarrow \varphi}(\lambda)^{-1} \Phi(\omega) E_{\psi \rightarrow \omega}(\lambda),
$$

where $a$ and $\Phi$ are in $C^{\infty}\left(S^{n-1}\right)$, with pointwise operations and with the norm

$$
\|\mathfrak{B} ; \mathfrak{S}\|=\sup \left\{\left\|\mathfrak{B}(\lambda) ; B L_{2}\left(S^{n-1}\right)\right\| ; \lambda \in \mathbb{R}\right\} .
$$

This algebra appeared in Subsection 2.3.4. The mappings

$$
\begin{aligned}
& \pi(\lambda): \mathfrak{B} \mapsto \mathfrak{B}(\lambda): L_{2}\left(S^{n-1}\right) \rightarrow L_{2}\left(S^{n-1}\right), \quad \lambda \in \mathbb{R}, \\
& \pi(\varphi, \omega): \mathfrak{B} \mapsto \Phi(\varphi, \omega), \quad(\varphi, \omega) \in S^{n-1} \times S^{n-1},
\end{aligned}
$$

extend to irreducible representations of $\mathfrak{S}$. These representations are pairwise nonequivalent. Any irreducible representation of $\mathfrak{S}$ is equivalent to one of the representations (3.4) or (3.5).

We turn to the $J$-topology on the spectrum $\widehat{\mathfrak{S}}$. Denote by $\Sigma$ the union of the product $S^{n-1} \times S^{n-1}$ and the line $l=\mathbb{R}$. The correspondence $\pi(\varphi, \omega) \mapsto(\varphi, \omega), \pi(\lambda) \mapsto \lambda$, 
yields a bijection of $\widehat{\mathfrak{S}}$ onto $\Sigma$. We introduce a topology on $\Sigma$. If $(\varphi, \omega) \in S^{n-1} \times S^{n-1}$ and the inner product $\varphi \omega$ of unit vectors $\varphi$ and $\omega$ in $\mathbb{R}^{n}$ satisfies $\varphi \omega>0(<0)$, then a fundamental system of neighborhoods of the point $(\varphi, \omega)$ can be formed by the sets $\mathcal{V}(\varphi, \omega) \cup\{\lambda \in l: \lambda>N(<N)\}$, where $\mathcal{V}(\varphi, \omega)$ is a neighborhood of $(\varphi, \omega)$ in the product of spheres $S^{n-1} \times S^{n-1}$ and $N \in \mathbb{R}$. If $\varphi \omega=0$, a fundamental system of neighborhoods is formed by the sets $\mathcal{V}(\varphi, \omega) \cup \mathbb{R}$. A neighborhood of a point $\lambda \in l \subset \Sigma$ is an interval in $l$. The topology on $\widehat{\mathfrak{S}}$ induced by that on $\Sigma$ coincides with the $J$-topology and turns out to be not separated; $\widehat{\mathfrak{S}}$ is a $T_{0}$-space: at least one of any two points has a neighborhood not containing the other. (The facts about the algebra $\mathfrak{S}$ were proved in [13]; see also [11.)

Now (3.2) takes the form $\varnothing \subset \mathbb{R} \subset \Sigma=\widehat{\mathfrak{S}}$. We set $I_{0}=\{\mathfrak{A} \in \mathfrak{S}: \pi(x, \xi) \mathfrak{A}=0$ for all $\left.(x, \xi) \in S^{n-1} \times S^{n-1}\right\}$ and $I_{1}=\mathfrak{S}$. It is known 12 that $I_{0} \simeq C_{0}(\mathbb{R}) \otimes \mathcal{K} L_{2}\left(S^{n-1}\right)$ and $I_{1} / I_{0}=\mathfrak{S} / C_{0}(\mathbb{R}) \otimes \mathcal{K} L_{2}\left(S^{n-1}\right) \simeq C\left(S^{n-1} \times S^{n-1}\right)$. The composition series $0 \subset$ $I_{0} \subset I_{1}=\mathfrak{S}$ is solving. Note that the ideal $I_{0}$ is the intersection of the kernels of all one-dimensional representations of $\mathfrak{S}$, so that $I_{0} \simeq \operatorname{com} \mathfrak{S}$, where com $\mathfrak{S}$ is the ideal in $\mathfrak{S}$ spanned by the commutators of the elements in $\mathfrak{S}$. Therefore, the solving series can be rewritten in the form $0 \subset$ com $\mathfrak{S} \subset \mathfrak{S}$.

Example 3.10. The algebra $\mathcal{E}$ is spanned by the functions

$$
S^{m-n-1} \ni \theta \mapsto A(\theta)=F_{\eta \rightarrow x}^{-1} \Phi(\varphi, \eta, \theta) F_{y \rightarrow \eta} \in \mathcal{B} L_{2}\left(\mathbb{R}^{n}\right),
$$

where $\varphi=x^{(1)} /\left|x^{(1)}\right|, \Phi \in C^{\infty}\left(S^{n-1} \times\left(\mathbb{R}^{m} \backslash 0\right)\right)$, and the function $\xi \mapsto \Phi(\varphi, \xi)$ is homogeneous of zero degree. This algebra already appeared in Subsection 2.3.3 (see (2.18)); it was studied in [13.

Let $A(\cdot)$ be a function of the form (3.6). We introduce the following mappings:

1) $\pi(\theta): A \mapsto A(\theta) \in \mathcal{B} L_{2}\left(\mathbb{R}^{n}\right)$ for $\theta \in S^{m-n-1}$;

2) $\pi(\lambda): A(\cdot) \mapsto \mathfrak{A}(\lambda) \in \mathcal{B} L_{2}\left(S^{n-1}\right)$, where $\mathfrak{A}(\lambda)=E_{\omega \rightarrow \varphi}(\lambda)^{-1} \Phi(\varphi, \omega, 0) E_{\psi \rightarrow \omega}(\lambda)$ and $\lambda \in \mathbb{R}$;

3) $\pi(\varphi, \xi): A(\cdot) \mapsto \Phi(\varphi, \xi)$ for $(\varphi, \xi) \in S^{n-1} \times S^{m-1}$.

These mappings extend to irreducible pairwise nonequivalent representations of $\mathcal{E}$. Any irreducible representation of $\mathcal{E}$ is equivalent to one such representation.

Passing to the topology of the spectrum, we denote by $\Lambda$ the disjoint union of the sets $S^{m-n-1}, S^{n-1} \times S^{m-1}$, and $\mathbb{R}$ and introduce a topology on $\Lambda$. Typical neighborhoods (forming a fundamental system) of a point $\theta \in S^{m-n-1}$ in the space $\Lambda$ coincide with those in $S^{m-n-1}$. If $\xi=\left(\xi^{(1)}, \xi^{(2)}\right), \xi^{(1)}=\left(\xi_{1}, \ldots, \xi_{n}\right), \xi^{(2)}=\left(\xi_{n+1}, \ldots, \xi_{m}\right)$, and $\xi^{(2)} \neq 0$, then a neighborhood in $\Lambda$ of a point $(\varphi, \xi) \in S^{n-1} \times S^{m-1}$ is the union $(\mathcal{U}(\varphi) \times \mathcal{V}(\xi)) \cup \mathcal{W}(\theta)$, where $\theta=\xi^{(2)} /\left|\xi^{(2)}\right|$, and $\mathcal{U}(\varphi), \mathcal{V}(\xi)$, and $\mathcal{W}(\theta)$ are neighborhoods of the points $\varphi, \xi$, and $\theta$ in $S^{n-1}, S^{m-1}$, and $S^{m-n-1}$, respectively, and $\mathcal{V}(\xi) \cap\{\xi$ : $\left.\xi^{(2)}=0\right\}=\varnothing$. For $\xi=\left(\xi^{(1)}, 0\right), \varphi \xi^{(1)}=0$, a typical neighborhood of $(\varphi, \xi)$ is of the form $S^{m-n-1} \cup(\mathcal{U}(\varphi) \times \mathcal{V}(\xi)) \cup \mathbb{R}$; in the case where $\varphi \xi^{1} \gtrless 0$, the line $\mathbb{R}$ must be replaced with the set $\{\lambda \in \mathbb{R}: \lambda \gtrless N\}$ with arbitrary real $N$ (for $n=1$ the possibility $\varphi \xi^{1}=0$ is absent). A neighborhood of a point $\lambda \in \mathbb{R}$ is the union $(\lambda-\varepsilon, \lambda+\varepsilon) \cup S^{m-n-1}, \varepsilon>0$. Endowed with this topology, the $\Lambda$ becomes a $T_{0}$-space.

The correspondence $\pi(\theta) \mapsto \theta \in S^{m-n-1}, \pi(\lambda) \mapsto \lambda \in \mathbb{R}, \pi(\varphi, \xi) \mapsto(\varphi, \xi) \in S^{n-1} \times$ $S^{m-1}$ is a bijection of the spectrum $\widehat{\mathcal{E}}$ onto $\Lambda$. The topology carried by this bijection from $\Lambda$ over to $\widehat{\mathcal{E}}$ coincides with the $J$-topology.

We introduce the filtration

$$
\varnothing \subset S^{m-n-1} \subset S^{m-n-1} \cup \mathbb{R} \subset \Lambda=\widehat{\mathcal{E}}
$$


and set

$$
\begin{aligned}
& I_{0}:=J:=\{A \in \mathcal{E}: \pi(\lambda) A=0 \text { for all } \lambda \in \mathbb{R}\} \\
& \cap\left\{A \in \mathcal{E}: \pi(\varphi, \xi) A=0 \text { for all }(\varphi, \xi) \in S^{m-n-1} \times S^{m-1}\right\}, \\
& I_{1}:=\operatorname{com} \mathcal{E}=\left\{A \in \mathcal{E}: \pi(\varphi, \xi) A=0 \text { for all }(\varphi, \xi) \in S^{m-n-1} \times S^{m-1}\right\} .
\end{aligned}
$$

We consider the composition series $0 \subset I_{0} \subset I_{1} \subset I_{2}:=\mathcal{E}$. It is known [13] that

$$
\begin{aligned}
J & \simeq C\left(S^{m-n-1}\right) \otimes \mathcal{K} L_{2}\left(\mathbb{R}^{n}\right), \\
\operatorname{com} \mathcal{E} / J & \simeq C_{0}(\mathbb{R}) \otimes \mathcal{K} L_{2}\left(S^{n-1}\right), \\
\mathcal{E} / \operatorname{com} \mathcal{E} & \simeq C\left(S^{n-1} \times S^{m-1}\right) .
\end{aligned}
$$

Thus, the composition series $0 \subset J \subset \operatorname{com} \mathcal{E} \subset \mathcal{E}$ is solving.

3.1.4. The spectral topology of the algebra $\mathcal{A}$. We turn to the algebra $\mathcal{A}$ defined at the end of Subsection 2.1. The irreducible representations of $\mathcal{A}$ were listed in Theorem 2.4. First, we introduce a set parametrizing the spectrum of $\mathcal{A}$. Assume that $s_{0}, \ldots, s_{k}$ is a complete chain of strata of the partition $\mathcal{T}$ of $\mathcal{M}$ (see Subsection 2.1) and that $z^{0}, \ldots, z^{k}$ are variables related to this chain: $z^{0}$ runs over $Z_{0}=s_{0}$ and $z^{j}$ runs over the stratum $Z_{j}$ of the partition $\mathcal{T}\left(s_{0}, \ldots, s_{j-1}\right), 1 \leq j \leq k$. The stratum $s_{0}$ is a submanifold of the manifold $\mathcal{M}$, and the stratum $Z_{j}, 1 \leq j \leq k$, is a submanifold of the corresponding sphere. We set $\mathcal{Z}_{j}=Z_{0} \times \cdots \times Z_{j}$ for $j=0, \ldots, k$. A point $\left(z^{0}, \ldots, z^{j}\right)$ in $\mathcal{Z}_{j}$ indicates in $\mathcal{M}$ the direction of approach to the point $z^{0} \in s_{0}$ and, thereby, determines a $d_{j}$-dimensional subspace $T\left(s_{j} ; z^{0}, \ldots, z^{j}\right)$ of $T(\mathcal{M})_{z^{0}}$ tangent to the stratum $s_{j}$. Denote by $S^{*}\left(s_{j} ; z^{0}, \ldots, z^{j}\right)$ the unit sphere in the cotangent space $T^{*}\left(s ; z^{0}, \ldots, z^{j}\right)$. Let $S^{*}\left(\mathcal{Z}_{j}\right)$ stand for the induced bundle with base $\mathcal{Z}_{j}$ and fiber $S^{*}\left(s_{j} ; z^{0}, \ldots, z^{j}\right)$ over a point $\left(z^{0}, \ldots, z^{j}\right)$. We introduce the disjoint union $\Sigma$ of the sets

$$
S^{*}(\mathfrak{C}), \quad \bigcup_{(\mathcal{T})} \bigcup_{j=0}^{k} S^{*}\left(\mathcal{Z}_{j}\right), \quad \bigcup_{(\mathcal{T})} \bigcup_{j=0}^{k} \mathcal{Z}_{j} \times \mathbb{R},
$$

where $\mathfrak{C}$ is the compact set defined before formula (2.8), and the union $\bigcup_{(\mathcal{T})}$ is taken over all complete chains of strata in $\mathcal{T}$. Theorem 2.4 shows that the spectrum of the quotient algebra $\mathcal{A} / \mathcal{K} L_{2}(\mathcal{M})$ can be parametrized by the points of $\Sigma$. The spectrum of $\mathcal{A}$ turns out to be parametrized by the points of the set $e \cup \Sigma$, where the additional "point" $e$ corresponds to the identity representation of the algebra $\mathcal{A}$.

In what follows, we compare representations obtained by localization along distinct chains of strata. Let us explain how to do this. Assume that $s_{l^{0}}, \ldots, s_{l^{q}}$ is a chain consisting of some strata of a chain $s_{0}, \ldots, s_{j}, 0 \leq l^{0}<\cdots<l^{q}=j$. We say that the new chain is a subchain of the initial one. Now we want to describe which of the one-type representations $\pi\left(z^{0}, \ldots, z^{j} ; \theta^{0}, \ldots, \theta^{j}\right)$ and $\pi\left(\zeta^{0}, \ldots, \zeta^{q} ; \tau^{0}, \ldots, \tau^{q}\right)$ or $\pi\left(z^{0}, \ldots, z^{j} ; \lambda\right)$ and $\pi\left(\zeta^{0}, \ldots, \zeta^{q} ; \mu\right)$ should be viewed as close ones; here $\pi\left(z^{0}, \ldots, z^{j} ; \cdot\right)$ and $\pi\left(\zeta^{0}, \ldots, \zeta^{q} ; \cdot\right)$ are obtained by localization along the chain and the subchain, respectively.

Suppose a point $\zeta^{0} \in s_{l^{0}}$ belongs to a sufficiently small tubular neighborhood of the stratum $s_{0}$. For this point, we can define "cylindrical coordinates" $\left(z^{0}, r^{0}, \alpha^{0}\right)$, where $z^{0} \in s_{0}=Z_{0}, r^{0} \in \overline{\mathbb{R}}_{+}$, and $\alpha^{0} \in S^{m-d_{0}-1}$. For the points $\alpha^{0}$ in a tubular neighborhood of the stratum $\mathcal{Z}_{1}$ we have coordinates $\left(z^{1}, r^{1}, \alpha^{1}\right)$, and so on. For a couple $\left(\zeta^{0}, \zeta^{1}\right)$ we obtain a row $\left(z^{0}, r^{0}, \ldots, z^{p}, r^{p}, \zeta^{1}\right)$, where $p=l^{0}-1$ and $\zeta^{1} \in S^{m-d_{p}-1}$. Continuing this procedure, we rewrite the row $\left(\zeta^{0}, \ldots, \zeta^{q}\right)$ in the form $\left(z^{0}, r^{0}, \ldots, z^{j-1}, r^{j-1}, r^{j}\right)$, where $j=l^{q}, z^{j}=\zeta^{q} \in S^{m-d_{j-1}-1}$. Collections $\left(z_{0}^{0}, \ldots, z_{0}^{j}\right)$ and $\left(\zeta^{0}, \ldots, \zeta^{q}\right) \equiv$ $\left(z^{0}, r^{0}, \ldots, z^{j-1}, r^{j-1}, z^{j}\right)$ are said to be close if the nonnegative numbers $r^{0}, \ldots, r^{j-1}$ are sufficiently small and the points $z_{0}^{\nu}$ and $z^{\nu}$ are close as points of the same stratum 
$Z_{\nu}(\nu=0, \ldots, j)$. Furthermore, two points $\left(\theta^{0}, \ldots, \theta^{j}\right)$ and $\left(\tau^{0}, \ldots, \tau^{q}\right)$ with $\left|\theta^{0}\right|^{2}+\cdots$ $+\left|\theta^{j}\right|^{2}=1$ and $\left|\tau^{0}\right|^{2}+\cdots+\left|\tau^{q}\right|^{2}=1$ belong to $d_{j}$-dimensional spheres; if $\left(z_{0}^{0}, \ldots, z_{0}^{j}\right)$ and $\left(\zeta^{0}, \ldots, \zeta^{q}\right)$ are close, then the spheres can be identified in a natural way with a "standard" sphere (local trivialization). Two representations $\pi\left(z^{0}, \ldots, z^{j} ; \theta^{0}, \ldots, \theta^{j}\right)$ and $\pi\left(\zeta^{0}, \ldots, \zeta^{q} ; \tau^{0}, \ldots, \tau^{q}\right)$ are said to be close if the collections $\left(z_{0}^{0}, \ldots, z_{0}^{j}\right)$ and $\left(\zeta^{0}, \ldots, \zeta^{q}\right)$ are close (in the sense mentioned above) and the points $\left(\theta^{0}, \ldots, \theta^{j}\right)$ and $\left(\tau^{0}, \ldots, \tau^{q}\right)$ differ little in the standard sphere $S^{d_{j}}$. The proximity of representations $\pi\left(z^{0}, \ldots, z^{j} ; \lambda\right)$ and $\pi\left(\zeta^{0}, \ldots, \zeta^{q} ; \mu\right)$ can be described in a similar way.

We introduce a topology on the set $e \cup \Sigma$ by indicating typical neighborhoods that form a fundamental system. (Recall that the set $e \cup \Sigma$ parametrizes the spectrum of the algebra $\mathcal{A}$.) By $\mathcal{U}(x ; T)$ we denote an arbitrary neighborhood of a point $x$ in a topological space $T$.

Neighborhoods of the points in $S^{*}\left(\mathcal{Z}_{j}\right)$. Let $\left(z^{0}, \ldots, z^{j} ; \theta^{0}, \ldots, \theta^{j}\right) \in S^{*}\left(\mathcal{Z}_{j}\right), 0 \leq$ $j \leq k$, where $\theta^{j} \in \mathbb{R}^{d_{j}-d_{j-1}}, d_{-1}=0$, and $\left|\theta^{0}\right|^{2}+\cdots+\left|\theta^{j}\right|^{2}=1$. If $\theta^{0} \neq 0$, then a typical neighborhood $\mathcal{U}\left(\left(z^{0}, \ldots, z^{j} ; \theta^{0}, \ldots, \theta^{j}\right) ; \Sigma\right)$ contains the sets

$$
\mathcal{U}\left(\left(z^{0}, \ldots, z^{q}\right) ; \mathcal{Z}_{q}\right) \times \mathcal{U}\left(\eta^{q} ; S^{d_{q}-1}\right)
$$

for $q=0, \ldots, j$, where $\eta^{q}=\left(\theta^{0}, \ldots, \theta^{q}\right) /\left(\left|\theta^{0}\right|^{2}+\cdots+\left|\theta^{q}\right|^{2}\right)^{1 / 2} \in S^{d_{q}-1}$; also, this neighborhood contains all sufficiently close representations of the same types obtained by localization along subchains. (We write the part of $S^{*}\left(\mathcal{Z}_{j}\right)$ over a "sufficiently small" neighborhood $\mathcal{U}\left(\left(z^{0}, \ldots, z^{q}\right) ; \mathcal{Z}_{q}\right)$ as the product (3.7).)

Now, let $\theta^{0}=0, \ldots, \theta^{i}=0, \theta^{i+1} \neq 0$. A neighborhood $\mathcal{U}\left(\left(z^{0}, \ldots, z^{j} ; \theta^{0}, \ldots, \theta^{j}\right) ; \Sigma\right)$ consists of the sets (3.7) for $q=i+1, \ldots, j$, the products

$$
\begin{array}{ll}
\mathcal{U}\left(\left(z^{0}, \ldots, z^{p}\right) ; \mathcal{Z}_{p}\right) \times S^{d_{p}-1}, & p=0, \ldots, i, \\
\mathcal{U}\left(\left(z^{0}, \ldots, z^{r}\right) ; \mathcal{Z}_{r}\right) \times \mathbb{R}, & r=0, \ldots, i-1,
\end{array}
$$

and the product

$$
\mathcal{U}\left(\left(z^{0}, \ldots, z^{i}\right) ; \mathcal{Z}_{i}\right) \times\{\lambda \in \mathbb{R}: \lambda \lessgtr N\}
$$

in the case where $z^{i+1} \theta^{i+1} \lessgtr 0$, or the product

$$
\mathcal{U}\left(\left(z^{0}, \ldots, z^{i}\right) ; \mathcal{Z}_{i}\right) \times \mathbb{R}
$$

for $z^{i+1} \theta^{i+1}=0$; here $N$ is an arbitrary real number and $z^{i+1} \theta^{i+1}$ is the projection of $\theta^{i+1}$ to the unit vector $z^{i+1}$. (The vector $z^{i+1} \in \mathbb{R}^{m-d_{i}}$ is directed from the coordinate origin to a point in $Z_{i+1}$, and $\theta^{i+1}$ lies in the space spanned by $z^{i+1}$ and the space tangent to the stratum $Z_{i+1}$ at the point $z^{i+1}$.)

Neighborhoods of the points that belong to $\mathcal{Z}_{j} \times \mathbb{R}$. A typical neighborhood $\mathcal{U}\left(\left(z^{0}, \ldots, z^{j} ; \lambda\right) ; \Sigma\right)$ is the union of the sets (3.8) and (3.9) for $i=j$, the product $\mathcal{U}\left(\left(z^{0}, \ldots, z^{j}\right) ; \mathcal{Z}_{j}\right) \times \mathcal{U}(\lambda ; \mathbb{R})$, and close representations obtained by localization along subchains.

Neighborhoods of the points in $S^{*}(\mathfrak{C})$. Typical neighborhoods in $\Sigma$ of the inner points of the manifold $S^{*}(\mathfrak{C})$ are the same as in the cospherical bundle $S^{*}(\mathcal{M} \backslash|\mathcal{T}|)$. Consider the points $\left(z^{0}, \ldots, z^{j} ; \varphi^{j}, \xi\right) \in \mathcal{Z}_{j} \times\left(S^{m-d_{j}-1} \backslash\left|\mathcal{T}\left(s_{0}, \ldots, s_{j}\right)\right|\right) \times S^{m-1}$ on the boundary of $S^{*}(\mathfrak{C})$. We write such a point in the form $\left(z^{0}, \ldots, z^{j} ; \varphi^{j}, J\left(z^{0}\right)\left(\xi^{0}, \theta^{0}\right)\right)$, where $\xi^{i}=J\left(z^{0}, \ldots, z^{i+1}\right)\left(\xi^{i+1}, \theta^{i+1}\right), i=0, \ldots, j-1$; as before, $\theta^{i} \in \mathbb{R}^{d_{i}-d_{i-1}}, \xi^{j} \in \mathbb{R}^{m-d_{j}}$; the Jacobi matrices $J\left(z^{0}, \ldots, z^{i+1}\right)$ are defined by induction (see Subsection 2.2 there we assumed that $J\left(z^{0}\right)=1$ and wrote $J\left(z^{1}\right)$ instead of $\left.J\left(z^{0}, z^{1}\right)\right)$. In what follows the points $\left(z^{0}, \ldots, z^{j} ; \varphi^{j}, \xi\right)$ are denoted by $\left(z^{0}, \ldots, z^{j} ; \varphi^{j}, \xi^{j}, \theta^{0}, \ldots, \theta^{j}\right)$. From now on we tacitly assume that any neighborhood, apart from the representations listed explicitly, 
contains representations of the same type obtained by localization along subchains and close to those listed.

If $\theta^{0} \neq 0$, then a neighborhood in $\Sigma$ of a point $\left(z^{0}, \ldots, z^{j} ; \varphi^{j}, \xi^{j}, \theta^{0}, \ldots, \theta^{j}\right)$ is the union of the sets (3.7) for $q=0, \ldots, j$ and also the product

$$
\mathcal{U}\left(\left(z^{0}, \ldots, z^{i}\right) ; \mathcal{Z}_{i}\right) \times \mathcal{U}\left(\left(\varphi^{j}, \xi\right) ;\left(S^{m-d_{j}-1} \backslash\left|\mathcal{T}\left(s_{0}, \ldots, s_{j}\right)\right|\right) \times S^{m-1}\right)
$$

(in this case the second factor contains no points with $\theta^{0}=0$ ).

Assume that $\theta^{0}, \ldots, \theta^{i}=0, \theta^{i+1} \neq 0,0 \leq i \leq 1$. Then a typical neighborhood consists of the sets (3.8); the sets (3.7) for $q=i+1, \ldots, j$; the sets (3.9); and the product

$$
\mathcal{U}\left(\left(z^{0}, \ldots, z^{i}\right) ; \mathcal{Z}_{i}\right) \times\{\lambda \in \mathbb{R}: \lambda \lessgtr N\}
$$

in the case where $z^{i+1} \theta^{i+1} \lessgtr 0$, or

$$
\mathcal{U}\left(\left(z^{0}, \ldots, z^{i}\right) ; \mathcal{Z}_{i}\right) \times \mathbb{R}
$$

in the case where $z^{i+1} \theta^{i+1}=0$.

Finally, suppose that $\theta^{0}=0, \ldots, \theta^{j}=0$. A neighborhood consists of the union of the sets (3.8) and (3.9) for $i=j$; the product $\mathcal{U}\left(\left(z^{0}, \ldots, z^{i}\right) ; \mathcal{Z}_{i}\right) \times\{\lambda \in \mathbb{R}: \lambda \lessgtr N\}$ for $\varphi^{j} \xi^{j} \lessgtr 0 ;$ or $\mathcal{U}\left(\left(z^{0}, \ldots, z^{i}\right) ; \mathcal{Z}_{i}\right) \times \mathbb{R}$ for $\varphi^{j} \xi^{j}=0$.

The topology defined on $\Sigma$ by the above neighborhoods is not separated; $\Sigma$ is a $T_{0^{-}}$ space (at least one of any two points has a neighborhood not containing the other). Recall that the sets $\Sigma$ and $\left(\mathcal{A} / \mathcal{K} L_{2}(\mathcal{M})^{\wedge}\right.$ are related by the bijection established in Theorem 2.4. The next theorem was proved in [7].

Theorem 3.11. The topology on $\Sigma$ coincides with the Jacobson topology on the spectrum $\left(\mathcal{A} / \mathcal{K} L_{2}(\mathcal{M})\right)^{\wedge}$.

The topology on the spectrum of $\mathcal{A}$ can easily be obtained from Theorem 3.11. We introduce a topology on $\Sigma \cup e$. The point $e$ will be taken as an open set. By definition, a neighborhood in $\Sigma \cup e$ of any point $\pi \in \Sigma$ is a neighborhood of $\pi$ in $\Sigma$ supplemented with $e$.

Corollary 3.12. The topology on $\Sigma \cup e$ coincides with the Jacobson topology on the spectrum $\widehat{\mathcal{A}}$ of the algebra $\mathcal{A}$.

3.2. Solving series (formulation of the main theorem). The ideals of a solving series are described as the intersections of the kernels of some explicitly indicated irreducible representations of the algebra $\mathcal{A}$. First, we present an auxiliary composition series; in general, this series is not solving. In essence, to form such a series, we compare all the chains of strata of the partition $\mathcal{T}$ of $\mathcal{M}$. Then, complementing the auxiliary series with some new ideals, we obtain a solving series; for this we use arguments related to Examples 3.9 and 3.10 .

Let $\mathcal{T}$ be an admissible partition of the manifold $\mathcal{M}$. The union of all strata of dimension at most $j$ is called the $j$-dimensional skeleton of $\mathcal{T}$ and is denoted by $\mathcal{T}_{j}$. In general, the sequence $\varnothing=\mathcal{T}_{-1}, \mathcal{T}_{0}, \ldots, \mathcal{T}_{m-1}$ can contain coinciding elements. We form a subsequence $\mathcal{T}_{-1}, \mathcal{T}_{m_{1}}, \ldots, \mathcal{T}_{m_{k}}$, where $-1 \leq m_{k} \leq m-1$ and $\mathcal{T}_{m_{p}}=\mathcal{T}_{m_{q}}$ only for $p=q$. For this, moving from left to right, we include in the subsequence every element not encountered before. In what follows, instead of $\mathcal{T}_{m_{j}}$, we shall write simply $\mathcal{T}_{j}$, where $\operatorname{dim} \mathcal{T}_{j}=m_{j}$. We introduce all kinds of skeleton collections

$$
\left(\mathcal{T}_{j_{1}}, \ldots, \mathcal{T}_{j_{q}}\right), \quad 1 \leq j_{1}<\cdots<j_{q},
$$

of the partition $\mathcal{T}$. To simplify the notation once again, we write $\left(j_{1}, \ldots, j_{q}\right)$ instead of $\left(\mathcal{T}_{j_{1}}, \ldots, \mathcal{T}_{j_{q}}\right)$. Thus, in a row $\left(j_{1}, \ldots, j_{q}\right)$ the symbol $j_{k}$ stands for the skeleton of dimension $m_{j_{k}}$. 
The set of skeleton collections will be ordered as follows: $\left(j_{1}, \ldots, j_{q}\right) \prec\left(l_{1}, \ldots, l_{p}\right)$ if and only if either $j_{1}=l_{1}, \ldots, j_{h}=l_{h}$ and $j_{h+1}>l_{h+1}$, or $p>q$ and $j_{1}=l_{1}, \ldots, j_{q}=l_{q}$. It is clear that one of any two skeleton collections is subject to the other. Also, we introduce the empty collection $T_{\varnothing}$ and assume that $T_{\varnothing}$ is subject to the minimal collection $T_{\min }=(k)$. Two collections $T$ and $T^{\prime}$ are said to be neighboring if $T \prec T^{\prime}$ and there is no collection $T^{\prime \prime}$ such that $T \prec T^{\prime \prime} \prec T^{\prime}$. Thus, all collections form an increasing sequence of neighbors

$$
T_{\varnothing} \prec T_{1} \prec \cdots \prec T_{Q},
$$

where $T_{1}=T_{\min }=(k)$ and $T_{Q}=T_{\max }=(1, \ldots, k)$. The passage from $T=\left(j_{1}, \ldots, j_{q}\right)$ to the neighboring $T^{\prime} \succ T$ can be performed by one of the following operations:

(1) if $j_{q}<k$, then $T^{\prime}=\left(j_{1}, \ldots, j_{q}, k\right)$ (the number of components increases);

(2) if $j_{q}=k>1$ and either $j_{q-1}<k-1$ or $q=1$, then $T^{\prime}=\left(j_{1}, \ldots, j_{q-1}, k-1\right)$ (the number of components remains the same);

(3) if $j_{q}=k, j_{q-1}=k-1, \ldots, j_{q-p}=k-p>1$ and either $j_{q-p-1}<k-p-1$ or $q-p=1$, then $T^{\prime}=\left(j_{1}, \ldots, j_{q-p-1}, j_{q-p}-1\right)$ (the number of components reduces).

If none of the above operations is applicable, then this $T$ coincides with the maximal collection $(1, \ldots, k)$. The sequence (3.10) can be written explicitly:

$$
\begin{aligned}
T_{\varnothing} & \prec(k) \prec(k-1) \prec(k-1, k) \prec(k-2) \prec(k-2, k) \prec(k-2, k-1) \\
& \prec(k-2, k-1, k) \prec(k-3) \prec \cdots \prec(1,2, \ldots, k) .
\end{aligned}
$$

With every collection $T_{\nu}$ in (3.10), we associate an ideal $\mathcal{A}\left(T_{\nu}\right)$ in $\mathcal{A}$. For that we need some notation. Let $I$ be the intersection of the kernels of all one-dimensional representations of the algebra $\mathcal{A}$,

$$
I=\bigcap \operatorname{ker} \pi_{1}(x, \xi)
$$

where $(x, \xi)$ runs over the cospherical bundle $S^{*}(\mathfrak{C}), x \in \mathfrak{C}$, and $\xi \in S^{*}(\mathfrak{C})_{x}$; see (2.10). We denote the intersection of the kernels of all representations of the form (2.8) by $\Theta\left(T_{\varnothing}\right)$,

$$
\Theta\left(T_{\varnothing}\right)=\bigcap_{\backslash T_{\varnothing}} \operatorname{ker} \pi\left(z^{0}, \ldots, z^{j} ; \theta^{0}, \ldots, \theta^{j}\right) .
$$

Moreover, let $\Lambda\left(T_{\varnothing}\right)$ stand for the intersection of the kernels of all representations of the form (2.9),

$$
\Lambda\left(T_{\varnothing}\right)=\bigcap_{\backslash T_{\varnothing}} \operatorname{ker} \pi\left(z^{0}, \ldots, z^{j} ; \lambda\right)
$$

We set

$$
\mathcal{A}\left(T_{\varnothing}\right)=I \cap \Theta\left(T_{\varnothing}\right) \cap \Lambda\left(T_{\varnothing}\right) .
$$

Thus, $\mathcal{A}\left(T_{\varnothing}\right)$ is the intersection of the kernels of all irreducible representations of $\mathcal{A}$ except for the identity representation.

We introduce the ideal $\Theta\left(T_{1}\right)$ for $m_{k}>0$ as the intersection of the kernels of all representations (2.8) except for those of the form $\pi\left(z^{0} ; \theta^{0}\right)$, where a point $z^{0}$ runs over all strata $s$ of dimension $m_{k}$, and $\left(z^{0} ; \theta^{0}\right)$ runs over the cospherical bundle $S^{*}(s)$ over each stratum $s$. We write $\Theta\left(T_{1}\right)$ in the form

$$
\Theta\left(T_{1}\right)=\bigcap_{\backslash T_{1}} \operatorname{ker} \pi\left(z^{0}, \ldots, z^{j} ; \theta^{0}, \ldots, \theta^{j}\right)
$$

$\left(\backslash T_{1}\right.$ indicates the representations that are absent in the intersection considered). The representations $\pi\left(z^{0} ; \theta^{0}\right)$ with $z^{0} \in s$ are not defined for the zero-dimensional strata 
$s$ (however, in general, the representations $\pi\left(z^{0}, z^{1} ; \theta^{1}\right)$ and similar ones make sense). Thus, for $m_{k}=0$ we obtain

$$
\Theta\left(T_{1}\right)=\Theta\left(T_{\varnothing}\right)
$$

In a similar way, we set

$$
\Lambda\left(T_{1}\right)=\bigcap_{\backslash T_{1}} \operatorname{ker} \pi\left(z^{0}, \ldots, z^{j} ; \lambda\right),
$$

assuming that the right-hand side of (3.18) is the intersection of the kernels of all representations (2.9) except for those of the form $\pi\left(z^{0} ; \lambda\right)$, where $z^{0}$ runs over all $m_{k}$-dimensional strata $s$, and $\lambda$ runs over the real axis.

Suppose that the ideals $\Theta(T), \Lambda(T)$ have already been defined for all $T$ in (3.10) such that $T \prec T_{\nu}$, and that these $\Theta(T), \Lambda(T)$ are given as the intersections of some primitive ideals, i.e., kernels of irreducible representations. We introduce the ideals $\Theta\left(T_{\nu}\right), \Lambda\left(T_{\nu}\right)$. Let $T_{\nu}=\left(j_{1}, \ldots, j_{q}\right)$. Consider all chains of strata $s_{1}, \ldots, s_{q}$ satisfying $s_{\alpha} \in \operatorname{st}\left(s_{\beta}\right)$ for $\alpha>\beta$ and $\operatorname{dim} s_{\alpha}=m_{j_{\alpha}}, \alpha=1, \ldots, q$. If there are no such chains, then we set

$$
\Theta\left(T_{\nu}\right)=\Theta\left(T_{\nu-1}\right), \quad \Lambda\left(T_{\nu}\right)=\Lambda\left(T_{\nu-1}\right) .
$$

Now assume that some chains as above exist and that $\pi\left(z^{1}, \ldots, z^{q} ; \theta^{1}, \ldots, \theta^{q}\right)$ are the representations obtained by localization along such a chain. Relations (3.11) show that the kernels of these representations participate in the intersection that determines the ideal $\Theta\left(T_{\nu-1}\right)$. Removing the kernels $\operatorname{ker} \pi\left(z^{1}, \ldots, z^{q} ; \theta^{1}, \ldots, \theta^{q}\right)$ of all such representations from the intersection mentioned above, we obtain the ideal $\Theta\left(T_{\nu}\right)$,

$$
\Theta\left(T_{\nu}\right)=\bigcap_{\backslash T_{\nu}} \operatorname{ker} \pi\left(z^{1}, \ldots, z^{q} ; \theta^{1}, \ldots, \theta^{q}\right) .
$$

Similarly, removing the kernels $\operatorname{ker} \pi\left(z^{0}, \ldots, z^{j} ; \lambda\right)$ from the intersection of ideals equal to $\Lambda\left(T_{\nu-1}\right)$, we obtain the ideal

$$
\Lambda\left(T_{\nu}\right)=\bigcap_{\backslash T_{\nu}} \operatorname{ker} \pi\left(z^{0}, \ldots, z^{j} ; \lambda\right) .
$$

Furthermore, if $T_{\nu+1}=\left(j_{1}\right)$ and $m_{j_{1}}=0$ (which means that the collection $T_{\nu+1}$ is represented by the zero-dimensional skeleton), then

$$
\Theta\left(T_{\nu+1}\right)=\Theta\left(T_{\nu}\right)
$$

for the same reason as in the case of (3.17). Continuing this process, we define the ideals $\Theta(T)$ and $\Lambda(T)$ for all collections $T$ in the sequence (3.10). In particular, $\Theta\left(T_{Q}\right)=\mathcal{A}$ and $\Lambda\left(T_{Q}\right)=\mathcal{A}$. Introduce the ideals

$$
\mathcal{A}(T):=I \cap \Theta(T) \cap \Lambda(T),
$$

where $I$ is the ideal (3.12). Obviously, if $T \prec T^{\prime}$, then $\Theta(T) \subset \Theta\left(T^{\prime}\right)$ and $\Lambda(T) \subset \Lambda\left(T^{\prime}\right)$, whence $\mathcal{A}(T) \subset \mathcal{A}\left(T^{\prime}\right)$. Among the ideals $\mathcal{A}(T)$, identical ones can occur; see (3.19). Let $\mathbf{T}_{0}:=T_{\varnothing}, \mathbf{T}_{1}:=T_{i_{1}}, \ldots, \mathbf{T}_{p-1}:=T_{i_{p-1}}, \mathbf{T}_{p}:=T_{Q}$ be a subsequence of the sequence (3.10) such that the composition series

$$
\mathcal{A}\left(\mathbf{T}_{0}\right) \subset \mathcal{A}\left(\mathbf{T}_{1}\right) \subset \cdots \subset \mathcal{A}\left(\mathbf{T}_{p}\right) \subset \mathcal{A}
$$

consists of all different ideals of the form $\mathcal{A}(T)$; note that always $i_{1}=1$, i.e., $\mathbf{T}_{1}=T_{1}=$ $T_{\min }=(k)$; see (3.10).

In general, the series (3.24) is not solving. To obtain a solving composition series, we introduce intermediate ideals $J\left(\mathbf{T}_{j}\right)$. For this, we consider neighboring ideals $\mathcal{A}\left(\mathbf{T}_{j}\right)$ and $\mathcal{A}\left(\mathbf{T}_{j+1}\right)$ in (3.24). By (3.23), we have $\mathcal{A}\left(\mathbf{T}_{j}\right)=I \cap \Theta\left(\mathbf{T}_{j}\right) \cap \Lambda\left(\mathbf{T}_{j}\right)$. Set

$$
\mathcal{J}\left(\mathbf{T}_{j}\right)=I \cap \Theta\left(\mathbf{T}_{j+1}\right) \cap \Lambda\left(\mathbf{T}_{j}\right)
$$


for $j=0, \ldots, p-1$. If the zero-dimensional skeleton $\mathcal{T}_{0}$ of the partition $\mathcal{T}$ is not empty, then for some $\nu$ we have

$$
\mathcal{J}\left(\mathbf{T}_{\nu}\right)=\mathcal{A}\left(\mathbf{T}_{\nu}\right)
$$

(see (3.22) ), while for $j \neq \nu$ we have the strict inclusions

$$
\mathcal{A}\left(\mathbf{T}_{j}\right) \subset \mathcal{J}\left(\mathbf{T}_{j}\right) \subset \mathcal{A}\left(\mathbf{T}_{j+1}\right) .
$$

Thus, if the skeleton $\mathcal{T}_{0}$ is empty, then (3.27) is true for all $j=0, \ldots, p-1$, and we obtain the composition series

$$
0 \subset \mathcal{A}\left(\mathbf{T}_{0}\right) \subset \mathcal{J}\left(\mathbf{T}_{0}\right) \subset \mathcal{A}\left(\mathbf{T}_{1}\right) \subset \mathcal{J}\left(\mathbf{T}_{1}\right) \subset \cdots \subset \mathcal{A}\left(\mathbf{T}_{p}\right) \subset \mathcal{A} .
$$

If $\mathcal{T}_{0} \neq \varnothing$, then, for $\nu$ as in (3.26), the series (3.28) contains the link $\mathcal{A}\left(\mathbf{T}_{\nu}\right) \subset \mathcal{A}\left(\mathbf{T}_{\nu+1}\right)$ instead of $\mathcal{A}\left(\mathbf{T}_{\nu}\right) \subset \mathcal{J}\left(\mathbf{T}_{\nu}\right) \subset \mathcal{A}\left(\mathbf{T}_{\nu+1}\right)$.

Let $Z_{0} \times \cdots \times Z_{q}$ be the submanifold defined for a chain of strata $s_{0}, \ldots, s_{q}$ as described at the beginning of Subsection 3.1.4. We say that a chain $s_{0}, \ldots, s_{q}$ is connected with a skeleton collection $\mathbf{T}_{\nu}=\left(j_{0}, \ldots, j_{q}\right)$ if $\operatorname{dim} s_{\alpha}=m_{j_{\alpha}}, \alpha=0, \ldots, q$ (compare with the description of formulas (3.19) and (3.20)). Denote by $\boldsymbol{\Upsilon}_{\nu}$ the union of manifolds of the form $Z_{0} \times \cdots \times Z_{q}$ corresponding to all chains connected with the collection $\mathbf{T}_{\nu}$. Finally, we set $\delta_{\nu}=\operatorname{dim} m_{j_{q}}$.

Theorem 3.13. The composition series (3.28) is solving. Moreover,

$$
\begin{aligned}
\mathcal{A}\left(\mathbf{T}_{0}\right) & \simeq \mathcal{K} L_{2}(\mathcal{M}), \\
\mathcal{A} / \mathcal{A}\left(\mathbf{T}_{p}\right) & \simeq C\left(S^{*}(\mathfrak{C})\right),
\end{aligned}
$$

where $C\left(S^{*}(\mathfrak{C})\right)$ is the cospherical bundle over the compact set $\mathfrak{C}$ (see the description of $\mathfrak{C}$ before (2.8) ) and $\mathcal{A}\left(\mathbf{T}_{p}\right)=\operatorname{com} \mathcal{A}$. If the zero-dimensional skeleton $\mathcal{T}_{0}$ of the partition $\mathcal{T}$ of $\mathcal{M}$ is empty, then

$$
\mathcal{J}\left(\mathbf{T}_{j}\right) / \mathcal{A}\left(\mathbf{T}_{j}\right) \simeq C_{0}\left(S^{*}\left(\mathbf{\Upsilon}_{j}\right)\right) \otimes \mathcal{K} L_{2}\left(\mathbb{R}^{m-\delta_{j}}\right),
$$

where $j=0, \ldots, p-1$, and

$$
\mathcal{A}\left(\mathbf{T}_{j}\right) / \mathcal{J}\left(\mathbf{T}_{j-1}\right) \simeq C_{0}\left(\mathbf{\Upsilon}_{j} \times \mathbb{R}\right) \otimes \mathcal{K} L_{2}\left(S^{m-\delta_{j}-1}\right),
$$

where $j=1, \ldots, p$. If $\mathcal{T}_{0} \neq \varnothing$, then for the number $\nu$ as in (3.22), instead of the link $\mathcal{A}\left(\mathbf{T}_{\nu}\right) \subset \mathcal{J}\left(\mathbf{T}_{\nu}\right) \subset \mathcal{A}\left(\mathbf{T}_{\nu+1}\right)$, the series (3.28) contains $\mathcal{A}\left(\mathbf{T}_{\nu}\right) \subset \mathcal{A}\left(\mathbf{T}_{\nu+1}\right)$ and the two formulas (3.31) for $j=\nu$ and (3.32) for $j=\nu+1$ are replaced by the only relation

$$
\mathcal{A}\left(\mathbf{T}_{\nu+1}\right) / \mathcal{A}\left(\mathbf{T}_{\nu}\right) \simeq C_{0}\left(\left|\mathcal{T}_{0}\right| \times \mathbb{R}\right) \otimes \mathcal{K} L_{2}\left(S^{m-1}\right) .
$$

\subsection{Proof of Theorem 3.13 .}

3.3.1. Verification of formulas (3.29) and (3.30). First, we consider (3.29). Theorem 2.4 implies that all irreducible representations of the algebra $\mathcal{A}$, except for the identity one, vanish on the ideal $\mathcal{A}\left(\mathbf{T}_{0}\right)$. Since the algebra $\mathcal{A}$ is irreducible, so is the ideal $\mathcal{A}\left(\mathbf{T}_{0}\right)$. Moreover, this ideal contains some compact operators, for example, the commutators $[a, P]$, where $a \in C^{\infty}(\mathcal{M}), a \mid \mathcal{T}_{m-1}=0$, and $P$ is a $\psi \mathrm{DO}$ in $\Psi^{0}(\mathcal{M})$. Therefore, $\mathcal{K} L_{2}(\mathcal{M}) \subset \mathcal{A}\left(\mathbf{T}_{0}\right)$. Since the identity representation is the only irreducible representation of $\mathcal{A}\left(\mathbf{T}_{0}\right)$, we have $\mathcal{A}\left(\mathbf{T}_{0}\right) \simeq \mathcal{K} L_{2}(\mathcal{M})$.

Now we turn to (3.30). It was noticed before (3.23) that $\Theta\left(\mathbf{T}_{p}\right)=\Lambda\left(\mathbf{T}_{p}\right)=\mathcal{A}$. Recall that $\mathbf{T}_{p}=T_{Q}$ and therefore, by (3.23), $\mathcal{A}\left(\mathbf{T}_{p}\right)=I$, where $I$ is the intersection of the kernels of all one-dimensional representations of $\mathcal{A}$. Hence, $\mathcal{A}\left(\mathbf{T}_{p}\right)=\operatorname{com} \mathcal{A}$. It remains to use the fact that, by Theorem 2.4 the list (2.10) contains all one-dimensional representations of $\mathcal{A}$. 
3.3.2. Verification of formulas (3.31). First we treat the case where $j=0$,

$$
\mathcal{J}\left(\mathbf{T}_{0}\right) / \mathcal{A}\left(\mathbf{T}_{0}\right) \simeq C_{0}\left(S^{*}\left(\mathbf{\Upsilon}_{0}\right)\right) \otimes \mathcal{K} L_{2}\left(\mathbb{R}^{m-\delta_{0}}\right) ;
$$

here $\mathcal{J}\left(\mathbf{T}_{0}\right)=I \cap \Theta\left(\mathbf{T}_{1}\right) \cap \Lambda\left(\mathbf{T}_{0}\right)$, the ideal $\Theta\left(\mathbf{T}_{1}\right)=\Theta\left(T_{1}\right)$ is defined by (3.16) (for $m_{k}>0$, which is assumed to be the case for the time being), and $\Lambda\left(\mathbf{T}_{0}\right)$ is given by (3.14). Thus, the spectrum $\mathcal{J}\left(\mathbf{T}_{0}\right)^{\wedge}$ consists of representations of the form $\pi\left(z^{0} ; \theta^{0}\right)$, where $z^{0}$ runs over the union $\boldsymbol{\Upsilon}_{0}$ of all strata of (maximal) dimension $m_{k}$, and $\left(z^{0} ; \theta^{0}\right)$ runs over the cospherical bundle $S^{*}\left(\mathbf{\Upsilon}_{0}\right)$.

We consider the algebra $\pi\left(z^{0} ; \theta^{0}\right) \mathcal{A}$ for a fixed point $\left(z^{0} ; \theta^{0}\right)$. Recall that all irreducible representations of $\mathcal{A}$, except for the identity, turn out to be representations of the quotient algebra $\mathcal{A} / \mathcal{K} L_{2}(\mathcal{M})$. The localization procedure in Subsection 2.3 .3 shows that any irreducible representation of $\pi\left(z^{0} ; \theta^{0}\right) \mathcal{A}$ (distinct from the identity representation) takes each operator $\pi\left(z^{0} ; \theta^{0}\right) A$ with $A \in \mathcal{J}\left(\mathbf{T}_{0}\right)$ to zero. The algebra spanned by the operators $\pi\left(z^{0} ; \theta^{0}\right) A$ with $A \in \mathcal{J}\left(\mathbf{T}_{0}\right)$ contains the ideal $\mathcal{K} L_{2}\left(\mathbb{R}_{z^{0}}^{m-m_{k}}\right)$, which can be verified with the help of Propositions 2.12 and 2.13 as well as the inclusion $\mathcal{K} L_{2}\left(\mathbb{R}^{n}\right) \subset \mathcal{L}(\theta)$ obtained before Proposition 2.14. Therefore, $\pi\left(z^{0} ; \theta^{0}\right) \mathcal{J}\left(\mathbf{T}_{0}\right)=\mathcal{K} L_{2}\left(\mathbb{R}_{z^{0}}^{m-m_{k}}\right)$ (we use the same notation for the space $\mathbb{R}_{z^{0}}^{m-m_{k}}$ and its pull-back to the fiber $S^{*}\left(\mathbf{\Upsilon}_{0}\right)_{z^{0}}$; the same applies to the notation $S_{z^{0}}^{m-m_{k}-1}$ ).

We introduce a continuous field of elementary algebras on $S^{*}\left(\boldsymbol{\Upsilon}_{0}\right)$. For this, with every point $\left(z^{0} ; \theta^{0}\right) \in S^{*}\left(\boldsymbol{\Upsilon}_{0}\right)$ we associate the algebra $\mathcal{K} L_{2}\left(\mathbb{R}_{z^{0}}^{m-m_{k}}\right)$. Any element $A$ of the ideal $\mathcal{J}\left(\mathbf{T}_{0}\right)$ gives rise to the vector field

$$
F_{A}:\left(z^{0} ; \theta^{0}\right) \mapsto \pi\left(z^{0} ; \theta^{0}\right) A \in \mathcal{K} L_{2}\left(\mathbb{R}_{z^{0}}^{m-m_{k}}\right) .
$$

Since all representations of $\pi\left(z^{0} ; \theta^{0}\right)$ vanish on the ideal $\mathcal{A}\left(\mathbf{T}_{0}\right)$, the field $F_{A}$ depends only on the residue class $[A]$ of an operator $A$ in $\mathcal{J}\left(\mathbf{T}_{0}\right) / \mathcal{A}\left(\mathbf{T}_{0}\right)$. On the set of vector fields of the form (3.34), we introduce the norm

$$
\left\|F_{A} ; \mathcal{F}_{0}\right\|=\sup \left\{\left\|\pi\left(z^{0} ; \theta^{0}\right) A ; \mathcal{B} L_{2}\left(\mathbb{R}_{z^{0}}^{m-m_{k}}\right)\right\| ;\left(z^{0} ; \theta^{0}\right) \in S^{*}\left(\Upsilon_{0}\right)\right\} ;
$$

in what follows $\mathcal{F}_{0}$ stands for the algebra of fields (3.34) with that norm. Assume also that $\Gamma_{0}$ is the set of vector fields that are limits of the fields in $\mathcal{F}_{0}$ with respect to the local uniform convergence. Taking the elements in $\Gamma_{0}$ as continuous vector fields, we introduce the continuous field $\left(\left\{\mathcal{K} L_{2}\left(\mathbb{R}_{z^{0}}^{m-m_{k}}\right)\right\}, \Gamma_{0}\right)$ of elementary algebras on $S^{*}\left(\boldsymbol{\Upsilon}_{0}\right)$.

By Theorem 2.4 the spectrum of $\mathcal{J}\left(\mathbf{T}_{0}\right) / \mathcal{A}\left(\mathbf{T}_{0}\right)$ can be parametrized by the points of the set $S^{*}\left(\mathbf{\Upsilon}_{0}\right)$. Therefore, for the residue class $[A] \in \mathcal{J}\left(\mathbf{T}_{0}\right) / \mathcal{A}\left(\mathbf{T}_{0}\right)$ of the operator $A \in \mathcal{J}\left(\mathbf{T}_{0}\right)$ we have

$$
\|[A]\|=\sup \left\{\left\|\pi\left(z^{0} ; \theta^{0}\right) A ; \mathcal{B} L_{2}\left(\mathbb{R}_{z^{0}}^{m-m_{k}}\right)\right\| ;\left(z^{0} ; \theta^{0}\right) \in S^{*}\left(\boldsymbol{\Upsilon}_{0}\right)\right\} .
$$

Hence, the mapping $[A] \mapsto F_{A}$ is an isomorphism

$$
\mathcal{J}\left(\mathbf{T}_{0}\right) / \mathcal{A}\left(\mathbf{T}_{0}\right) \simeq \mathcal{F}_{0}
$$

of $C^{*}$-algebras. Theorem 3.11 implies that the Jacobson topology on the spectrum $\left(\mathcal{J}\left(\mathbf{T}_{0}\right) / \mathcal{A}\left(\mathbf{T}_{0}\right)\right)^{\wedge}$ coincides with the topology of the cospherical bundle $S^{*}\left(\mathbf{\Upsilon}_{0}\right)$; i.e., the space $\left(\mathcal{J}\left(\mathbf{T}_{0}\right) / \mathcal{A}\left(\mathbf{T}_{0}\right)\right)^{\wedge}$ is finite-dimensional, Hausdorff, and locally compact. The set $\left\{\pi \in\left(\mathcal{J}\left(\mathbf{T}_{0}\right) / \mathcal{A}\left(\mathbf{T}_{0}\right)\right)^{\wedge}:\|\pi A\| \geq \alpha\right\}$ is compact for any $\alpha>0$ and any operator $A \in$ $\mathcal{J}\left(\mathbf{T}_{0}\right)$ (see [19, 3.3.7]). This means that every vector field $F_{A}, S^{*}\left(\boldsymbol{\Upsilon}_{0}\right) \ni \pi \mapsto \pi(A)$, tends to zero at infinity. The algebra determined by the continuous field $\left(\left\{\mathcal{K} L_{2}\left(\mathbb{R}_{z^{0}}^{m-m_{k}}\right)\right\}, \Gamma_{0}\right)$ is isomorphic to $\mathcal{F}_{0}$ by [19, 10.5.4].

The field $\left(\left\{\mathcal{K} L_{2}\left(\mathbb{R}_{z^{0}}^{m-m_{k}}\right)\right\}, \Gamma_{0}\right)$ is locally trivial; as local trivializations, one can use those of the normal bundle over $\boldsymbol{\Upsilon}_{0}$. (In connection with the normal bundle over $\boldsymbol{\Upsilon}_{j}$, see the definition of the fiber $\mathbb{R}^{m-d_{q}}\left(z^{0}, \ldots, z^{q}\right)$ over a point $\left(z^{0}, \ldots, z^{q}\right) \in \mathcal{Z}_{q}=Z_{0} \times \cdots \times Z_{q}$ 
given before Theorem 2.4) Proposition 3.6 provides the triviality of this field. Therefore, $\mathcal{F}_{0} \simeq C_{0}\left(S^{*}\left(\boldsymbol{\Upsilon}_{0}\right)\right) \otimes \mathcal{K} L_{2}\left(\mathbb{R}^{m-m_{k}}\right)$. Using (3.35), we obtain (3.31) for $j=0$.

Now, assume that $m_{k}=0$; then $k=1$ and the series (3.28) has the form $0 \subset \mathcal{A}\left(\mathbf{T}_{0}\right) \subset$ $\mathcal{A}\left(\mathbf{T}_{1}\right) \subset \mathcal{A}$. Thus, instead of (3.31) for $j=0$ and (3.32) for $j=1$, we need to prove the isomorphism

$$
\mathcal{A}\left(\mathbf{T}_{1}\right) / \mathcal{A}\left(\mathbf{T}_{0}\right) \simeq C_{0}\left(\left|\mathcal{T}_{0}\right| \times \mathbb{R}\right) \otimes \mathcal{K} L_{2}\left(S^{m-1}\right) ;
$$

here $\mathcal{A}\left(\mathbf{T}_{1}\right)=I=\operatorname{com} \mathcal{A}, \mathcal{A}\left(\mathbf{T}_{0}\right)=I \cap \Lambda\left(\mathbf{T}_{0}\right), \Lambda\left(\mathbf{T}_{0}\right)=\bigcap_{(z, \lambda)} \operatorname{ker} \pi(z ; \lambda), \lambda$ runs over the real axis, and $z$ runs over the finite set $\left|\mathcal{T}_{0}\right|$. By (3.29), we have $\mathcal{A}\left(\mathbf{T}_{0}\right)=\mathcal{K} L_{2}(\mathcal{M})$.

Consider functions of the form $\mathbb{R} \ni \lambda \mapsto \pi(z ; \lambda) A \in \mathcal{B} L_{2}\left(S^{m-1}\right)$, where $A \in \mathcal{A}\left(\mathbf{T}_{1}\right)$ and $z$ is a fixed point in $\left|\mathcal{T}_{0}\right|$. Since the representations $\pi(z ; \lambda)$ vanish on the ideal $\mathcal{A}\left(\mathbf{T}_{0}\right)$, the function $\pi(z ; \cdot) A$ depends only on the residue class $[A]$ in $\mathcal{A}\left(\mathbf{T}_{1}\right) / \mathcal{A}\left(\mathbf{T}_{0}\right)$. Such a function is an element of the algebra $\mathfrak{S}$ in Example 3.9 (for $n=m$ ). The definition of the ideal $\mathcal{A}\left(\mathbf{T}_{1}\right)$ shows that the ideal spanned by the functions in $\mathfrak{S}$ belongs to the kernel of every one-dimensional representation of $\mathfrak{S}$, and consequently, this ideal coincides with com $\mathfrak{S} \simeq C_{0}(\mathbb{R}) \otimes \mathcal{K} L_{2}\left(S^{m-1}\right)$. This leads to (3.36).

We turn to (3.31) for $j>0$. Assume that $\mathbf{T}_{j}=\left(j_{1}, \ldots, j_{q}\right)$, the ideals $\Theta\left(\mathbf{T}_{j}\right)$ and $\Lambda\left(\mathbf{T}_{j}\right)$ are as defined in (3.20) and (3.21), $\mathcal{A}\left(\mathbf{T}_{j}\right)=I \cap \Theta\left(\mathbf{T}_{j}\right) \cap \Lambda\left(\mathbf{T}_{j}\right)$, and $\mathcal{J}\left(\mathbf{T}_{j}\right)=I \cap \Theta\left(\mathbf{T}_{j+1}\right) \cap \Lambda\left(\mathbf{T}_{j}\right)$. Theorem 2.4 and the definitions of the ideals $\mathcal{A}\left(\mathbf{T}_{j}\right)$ and $\mathcal{J}\left(\mathbf{T}_{j}\right)$ imply that the spectrum $\left(\mathcal{J}\left(\mathbf{T}_{j}\right) / \mathcal{A}\left(\mathbf{T}_{j}\right)\right)^{\wedge}$ consists of the representations $\pi\left(z^{1}, \ldots, z^{q} ; \theta^{1}, \ldots, \theta^{q}\right)$, where $z:=\left(z^{1}, \ldots, z^{q}\right) \in \boldsymbol{\Upsilon}_{j}$ and $\theta:=\left(\theta^{1}, \ldots, \theta^{q}\right) \in S^{*}\left(\boldsymbol{\Upsilon}_{j}\right)_{z}$. For a fixed point $(z, \theta)$, we consider the algebra $\pi(z ; \theta) \mathcal{J}\left(\mathbf{T}_{j}\right)$. From Theorem 2.4 (and the localization procedure in its proof) it follows that the element $\pi(z ; \theta) A, A \in \mathcal{J}\left(\mathbf{T}_{j}\right)$, depends only on the residue class $[A] \in \mathcal{J}\left(\mathbf{T}_{j}\right) / \mathcal{A}\left(\mathbf{T}_{j}\right)$ and that the only irreducible representation (up to equivalence) of the quotient algebra $\mathcal{J}\left(\mathbf{T}_{j}\right) / \mathcal{A}\left(\mathbf{T}_{j}\right)$ is the identity. Moreover, as in the case where $j=0$, we have $\mathcal{K} L_{2}\left(\mathbb{R}^{m-\delta_{j}}\right) \subset \pi(z ; \theta) \mathcal{J}\left(\mathbf{T}_{j}\right)$ and $\pi(z ; \theta)\left(\mathcal{J}\left(\mathbf{T}_{j}\right) / \mathcal{A}\left(\mathbf{T}_{j}\right)\right)=\mathcal{K} L_{2}\left(\mathbb{R}^{m-\delta_{j}}\right)$. To complete the verification of (3.31) for $j>0$, it remains to repeat the proof of that formula for $j=0$ with obvious modifications.

3.3.3. Verification of formulas (3.32). The spectrum $\left(\mathcal{A}\left(\mathbf{T}_{j}\right) / \mathcal{J}\left(\mathbf{T}_{j-1}\right)\right)^{\wedge}$ can be parametrized by the points $(z ; \lambda)$, where $z=\left(z^{1}, \ldots, z^{q}\right) \in \mathbf{\Upsilon}_{j}$ and $\lambda \in \mathbb{R}$. The operator $\pi(z ; \lambda) A$ with $A \in \mathcal{A}\left(\mathbf{T}_{j}\right)$ depends only on the residue class $[A] \in \mathcal{A}\left(\mathbf{T}_{j}\right) / \mathcal{J}\left(\mathbf{T}_{j-1}\right)$. For a fixed $z$, the function $\lambda \mapsto \pi(z ; \lambda) A$ is an element of the algebra $\mathfrak{S}$ in Example 3.9 for $n=m-\delta_{j}$. The ideal in $\mathfrak{S}$ spanned by such functions with $A \in \mathcal{A}\left(\mathbf{T}_{j}\right)$ coincides with $\operatorname{com~} \mathfrak{S}(z) \simeq C_{0}(\mathbb{R}) \otimes \mathcal{K} L_{2}\left(S_{z}^{m-\delta_{j}-1}\right)$.

We introduce a field of elementary algebras on $\boldsymbol{\Upsilon}_{j} \times \mathbb{R}$. With every point $(z ;, \lambda) \in$ $\Upsilon_{j} \times \mathbb{R}$ we associate the algebra $\mathcal{K} L_{2}\left(S_{z}^{m-\delta_{j}-1}\right)$. Let $\mathcal{G}_{j}$ denote the algebra of vector fields of the form

$$
G_{A}:(z ; \lambda) \mapsto \pi(z ; \lambda) A \in \mathcal{K} L_{2}\left(S_{z}^{m-\delta_{j}-1}\right)
$$

endowed with the norm

$$
\left\|G_{a} ; \mathcal{G}_{j}\right\|=\sup \left\{\left\|\pi(z ; \lambda) A ; \mathcal{B} L_{2}\left(S_{z}^{m-\delta_{j}-1}\right)\right\| ;(z ; \lambda) \in \mathbf{\Upsilon}_{j} \times \mathbb{R}\right\} .
$$

Let $\Delta_{j}$ stand for the set of limits of the fields in $\mathcal{G}_{j}$ with respect to the local uniform convergence. Introduce the continuous field of elementary algebras $\left(\left\{\mathcal{K} L_{2}\left(S_{z}^{m-\delta_{j}-1}\right)\right\}, \Delta_{j}\right)$, where $\Delta_{j}$ plays the role of a set of continuous vector fields. The mapping $[A] \mapsto G_{A}$ realizes the isomorphism $\mathcal{A}\left(\mathbf{T}_{j}\right) / \mathcal{J}\left(\mathbf{T}_{j-1}\right) \simeq \mathcal{G}_{j}$. The algebra determined by the field $\left(\left\{\mathcal{K} L_{2}\left(S_{z}^{m-\delta_{j}-1}\right)\right\}, \Delta_{j}\right)$ is isomorphic to $\mathcal{G}_{j}$. The triviality of the field in question is provided by Proposition 3.6, as in the proof of (3.31). This leads to (3.32). 


\section{REFERENCES}

[1] A. S. Dynin, Inversion problem for singular integral operators: $C^{*}$-approach, Proc. Nat. Acad. Sci. U.S.A. 75 (1978), 4668-4670. MR0507929 (80c:46066)

[2] I. Ts. Gokhberg and N. Ya. Krupnik, The algebra generated by the one-dimensional singular integral operators with piecewise continuous coefficients, Funktsional. Anal. i Prilozhen. 4 (1970), no. 3, 2636; English transl. in Functional Anal. Appl. 4 (1970), no. 3. MR0270164 (42:5057)

[3] L. Boutet de Monvel, Boundary problems for pseudodifferential operators, Acta Math. 126 (1971), 11-51. MR0407904 (53:11674)

[4] R. G. Douglas and R. Howe, On the $C^{*}$-algebra of Toeplitz operators on the quarterplane, Trans. Amer. Math. Soc. 158 (1971), 203-217. MR0288591 (44:5787)

[5] B. A. Plamenevskiŭ and V. N. Senichkin, Solvable operator algebras, Algebra i Analiz 6 (1994), no. 5, 1-87; English transl., St. Petersburg Math. J. 6 (1995), no. 5, 895-968. MR.1318302 (95k:47038)

[6] C. Schochet, Topological methods for $C^{*}$-algebras. I. Spectral sequences, Pacific J. Math. 96 (1981), no. 1, 193-211. MR0634772 (84g:46105a)

[7] B. A. Plamenevskii and V. N. Senichkin, The spectrum of an algebra of pseudodifferential operators with piecewise smooth symbols, Izv. Akad. Nauk SSSR Ser. Mat. 53 (1989), no. 1, 147-178; English transl., Math. USSR-Izv. 34 (1990), no. 1, 147-179. MR0992982 (90d:58151)

[8] — On composition series in algebras of pseudodifferential operators and in algebras of Wiener-Hopf operators, Schrödinger Operators, Markov Semigroups, Wavelet Analysis, Operator Algebras, Math. Top., vol. 11, Akad. Verlag, Berlin, 1996, pp. 373-404. MR1409838 (97h:47050)

[9] _ Representations of $C^{*}$-algebras of pseudodifferential operators on piecewise smooth manifolds, Algebra i Analiz 13 (2001), no. 6, 124-174; English transl., St. Petersburg Math. J. 13 (2002), no. 6, 993-1032. MR1883841 (2002j:47128)

[10] _ On a class of pseudodifferential operators on $\mathbb{R}^{m}$ and on stratified manifolds, Mat. Sb. 191 (2000), no. 5, 109-142; English transl., Sb. Math. 191 (2000), no. 5-6, 725-757. MR1773771 (2002g:35219)

[11] B. A. Plamenevskiǔ, Algebras of pseudodifferential operators, Nauka, Moscow, 1986; English transl., Math. Appl. (Soviet Ser.), vol. 43, Kluwer Acad. Publ. Group, Dordrecht, 1989. MR0869253 (88d:47062) MR1026642 (90h:47098)

[12] B. A. Plamenevskil and V. N. Senichkin, The spectrum of $C^{*}$-algebras generated by pseudodifferential operators with discontinuous symbols, Izv. Akad. Nauk SSSR Ser. Mat. 47 (1983), no. 6, 1263-1284; English transl. in Math. USSR-Izv. 23 (1984), no. 3. MR0727755 (85c:47047)

[13] _ On representations of an algebra of pseudodifferential operators with multidimensional discontinuities in the symbols, Izv. Akad. Nauk SSSR Ser. Mat. 51 (1987), no. 4, 833-859; English transl., Math. USSR-Izv. 31 (1988), no. 1, 143-169. MR0914862 (89h:47077)

[14] R. G. Douglas, Banach algebra techniques in operator theory, Pure Appl. Math., vol. 49, Acad. Press, New York-London, 1972. MR0361893 (50:14335)

[15] A. S. Dynin, Multivariable Wiener-Hopf operators. I. Representations, Integral Equations Operator Theory 9 (1986), no. 4, 537-569. MR0853628 (87m:47066)

[16] I. B. Simonenko, Convolution type operators in cones, Mat. Sb. (N. S.) 74 (1967), no. 2, 298-313; English transl. in Math. USSR-Sb. 3 (1967). MR0222723 (36:5773)

[17] V. N. Senichkin, The spectrum of an algebra of pseudodifferential operators on a manifold with smooth edges, Algebra i Analiz 8 (1996), no. 6, 105-147; English transl., St. Petersburg Math. J. 8 (1997), no. 6, 985-1013. MR 1458140 (98e:47080)

[18] H. O. Cordes, On compactness of commutators of multiplications and convolutions, and boundedness of pseudodifferential operators, J. Funct. Anal. 18 (1975), no. 2, 115-131. MR0377599 $(51: 13770)$

[19] J. Dixmier, Les $C^{*}$-algèbres et leurs représentations, Cahiers Sci., vol. 29, Gauthier-Villars, Paris, 1969. MR0246136 (39:7442)

Department of Mathematical Physics, Physics Institute, St. Petersburg State University, Ulyanovskaya 1, St. Petersburg 198504, Russia

E-mail address: boris.plamen@gmail.com

Received 20/AUG/2008

Translated by THE AUTHOR 